Portland State University

PDXScholar

Fall 1-4-2013

\title{
Capillary Phenomena: Investigations in Compressed Bubble Migration, Geometric Wetting, and Blade- Bound Droplet Stability
}

William Henry Blackmore

Portland State University

Follow this and additional works at: https://pdxscholar.library.pdx.edu/open_access_etds

Part of the Mechanical Engineering Commons

Let us know how access to this document benefits you.

Recommended Citation

Blackmore, William Henry, "Capillary Phenomena: Investigations in Compressed Bubble Migration, Geometric Wetting, and Blade-Bound Droplet Stability" (2013). Dissertations and Theses. Paper 651. https://doi.org/10.15760/etd.651

This Thesis is brought to you for free and open access. It has been accepted for inclusion in Dissertations and Theses by an authorized administrator of PDXScholar. Please contact us if we can make this document more accessible: pdxscholar@pdx.edu. 
Capillary Phenomena: Investigations in Compressed Bubble Migration, Geometric Wetting, and Blade-Bound Droplet Stability

\title{
by
}

\section{William Henry Blackmore}

A thesis submitted in partial fulfillment of the requirements for the degree of

\author{
Master of Science \\ in \\ Mechanical Engineering
}

Thesis committee:

Mark M. Weislogel, Chair

Derek Tretheway

Raúl Bayoán Cal

\section{Portland State University}

2012 


\begin{abstract}
Capillary flows continue to be important in numerous spacecraft systems where the effective magnitude of the gravity vector is approximately one millionth that of normal Earth gravity. Due to the free fall state of orbiting spacecraft, the effects of capillarity on the fluid systems onboard can dominate the fluid behavior over large length scales. In this research three investigations are pursued where the unique interplay between surface tension forces, wetting characteristics, and system geometry control the fluid behavior, whether in large systems aboard spacecraft, or micro-scale systems on Earth.
\end{abstract}

First, efforts in support of two International Space Station (ISS) experiments are reported. A description of the development of a new NASA ground station at Portland State University is provided along with descriptions of astronaut training activities for the proper operation of four handheld experiments currently in orbit as part of the second iteration of the Capillary Flow Experiments (CFE-2). Concerning the latter, seven more vessels are expected to be launched to the ISS shortly. Analysis of the data alongside numerical simulations shows excellent agreement with theory, and a new intuitive method of viewing critical wetting angles and fluid bulk shift phenomena is offered.

Secondly, during the CFE-2 space experiments, unplanned peripheral observations revealed that, on occasion, rapidly compressed air bubbles migrate along paths with vector components common to the residual acceleration onboard the ISS. Unexpectedly however, the migration velocities could be shown to be up to three orders of magnitude greater than the appropriate Stokes flow limit! Likely mechanisms are explored analytically and experimentally while citing prior theoretical works that may 
have anticipated such phenomena. Once properly understood, compressed bubble migration may be used as an elegant method for phase separation in spacecraft systems or microgravity-based materials manufacturing.

Lastly, the stability of drops on surfaces is important in a variety of natural and industrial processes. So called 'wall-edge-vertex bound drops' (a.k.a. drops on blade tips or drops on leaf tips which they resemble) are explored using a numerical approach which applies the Surface Evolver algorithm through implementation of a new file layer and a multi-parameter sweep function. As part of a recently open sourced SE-FIT software, thousands of critical drop configurations are efficiently computed as functions of contact angle, blade edge vertex half-angle, and g-orientation. With the support of other graduate students, simple experiments are performed to benchmark the computations which are then correlated for ease of application. It is shown that sessile, pendant, and wall-edge bound drops are only limiting cases of the more generalized blade-bound drops, and that a ubiquitous 'dry leaf tip' is observed for a range of the critical geometric and wetting parameters. 


\section{Table of Contents}

$\begin{array}{lc}\text { Abstract } & \mathrm{i} \\ \text { List of Tables } & \mathrm{v} \\ \text { List of Figures } & \text { vi }\end{array}$

\section{Chapter 1}

Introduction

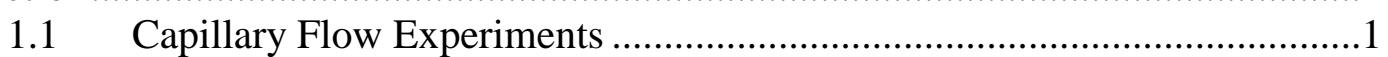

1.2 Compressed Bubble Migration .......................................................

$1.3 \quad$ Wall-Edge-Vertex-Bound Drops ......................................................

\section{Chapter 2}

Capillary Flow Experiments ................................................................................. 7

$2.1 \quad$ Background ................................................................................. 7

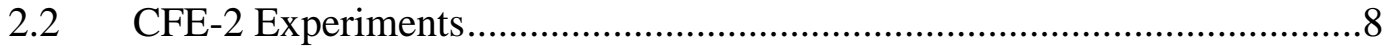

2.2.1 The Vane Gap Experiments ................................................ 8

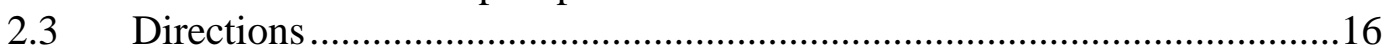

\section{Chapter 3}

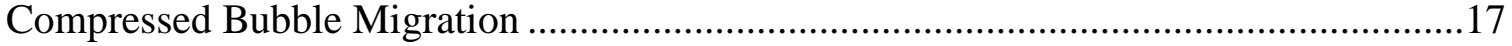

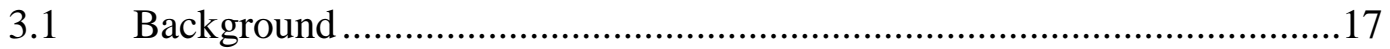

3.2 CFE-2 Experiments and Bubble Migration Observation .........................17

3.3 Compressed Bubble Migration Data.....................................................21

3.4 Preliminary Comparisons of Analytical and Numerical Predictions .........23

3.4.1 Local Acceleration Measurements .............................................23

3.4.2 Isothermal Stokes Flow Bubble Velocity Predictions ...................26

3.4.3 Isothermal CFD Bubble Velocity Predictions .............................27

3.4.4 Solutocapillary Mechanism ....................................................28

3.4.5 Thermocapillary Mechanism .................................................35

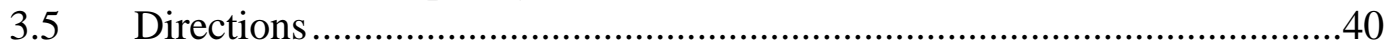

\section{Chapter 4}

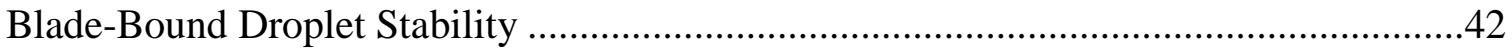

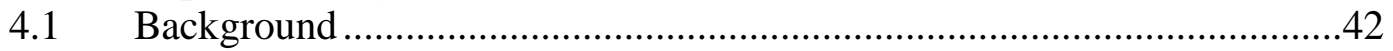

4.2 Description of Numerical Approach and Benchmarking ........................43

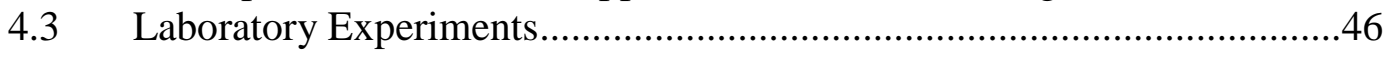

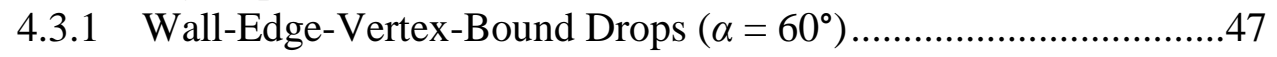

4.3.2 Residual Drops ..................................................................49

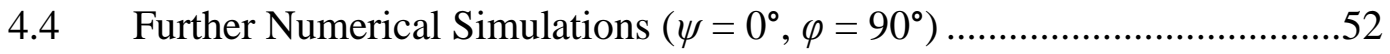

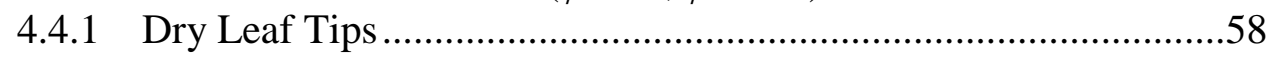

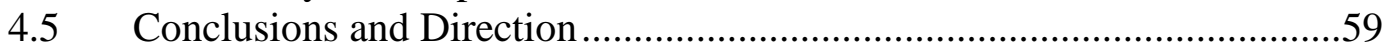

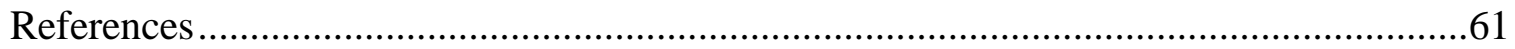


Appendix A

List of CFE-2 Experiments Performed to Date.

Appendix B

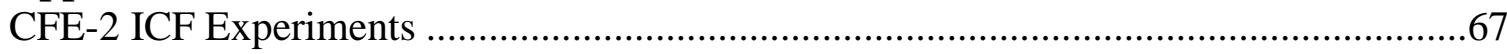

Appendix C

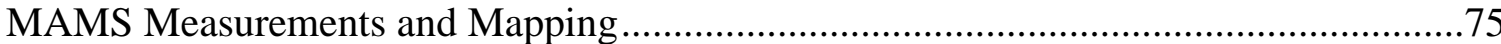




\section{List of Tables}

Table 1. VG1 critical angles with filled and unfilled perforations .................................11

Table 2. VG2 critical angles with filled and unfilled perforations .................................12

Table 3. Effective contact angles in degrees for both VG vessels .................................16

Table 4. CFE-2 compressed bubble migration observation chronology.........................19

Table 5. Pertinent ICF1/ICF2 container and fluid properties. ..................................20

Table 6. Migration velocities for each experiments and relevant dimensionless groups. .25

Table 7. Comparison of pendant drop simulations with numerical results. $\alpha=60^{\circ} \ldots \ldots . .45$

Table 8. Properties of the fluid used in the WEVB lab experiments..............................46 


\section{List of Figures}

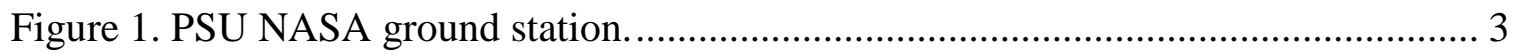

Figure 2. Solid model of VG1/VG2 with vane rotation schematic. ............................. 10

Figure 3. Comparison of VG1 SeW and SeDW with filled and unfilled perforations..... 13

Figure 4. VG1 vessel with and without bulk shift. .............................................. 14

Figure 5. Graphical representation of VG1 critical angles. ..................................... 14

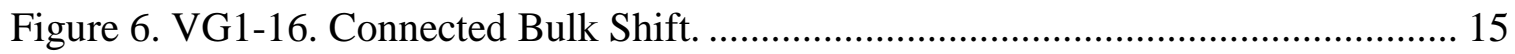

Figure 7. Solid perspective models of ICF1 and ICF2 vessels................................. 19

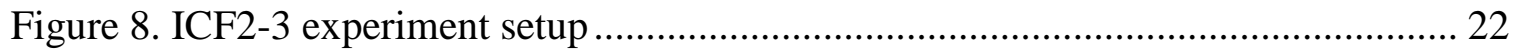

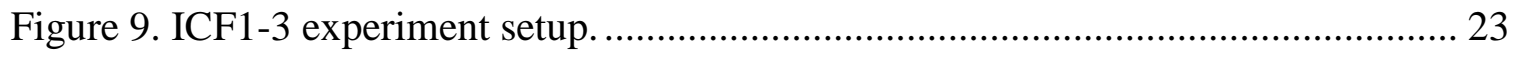

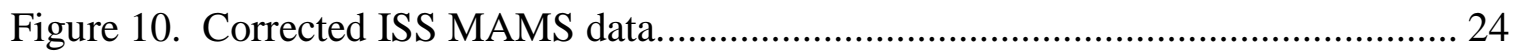

Figure 11. ISS experimental data for bubble migration is compared to isothermal Stokes

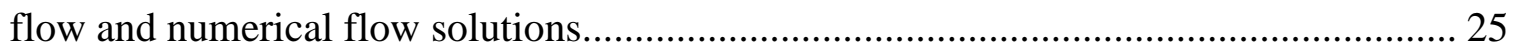

Figure 12. Transient surface tension measurement. .................................................. 29

Figure 13. Close up of the ICF1-8 transport tube system........................................ 30

Figure 14. ICF1-8 decompressed bubble migration data....................................... 31

Figure 15. Vacuum chamber experimental setup. ................................................ 32

Figure 16. Materials to adapt the KSV Sigma 702 tensiometer for use in a vacuum

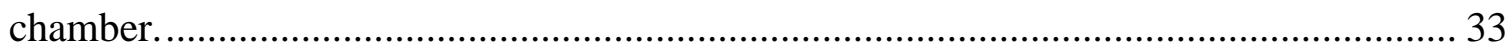

Figure 17. Dynamic vacuum chamber pressure data............................................... 34

Figure 18. Three hour vacuum chamber experiment data. ......................................... 35

Figure 19. Outcomes of the thermocapillary drag coefficient. ................................... 37 
Figure 20. Two configurations showing tube wall influence on surface temperature of a

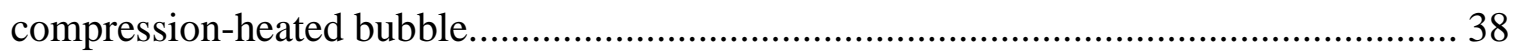

Figure 21. Sketch of WEVB configuration with numerical notation employed. ............ 44

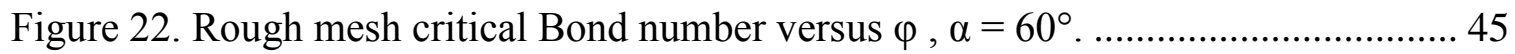

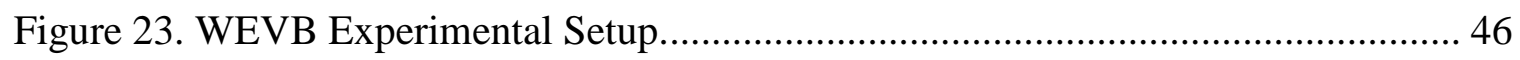

Figure 24. Comparison of SE drop profiles versus experimental results. ...................... 47

Figure 25. Finer mesh SE-FIT simulation versus experimental results for $\alpha=60^{\circ} . \ldots \ldots . .48$

Figure 26. SE simulation showing a Residual Drop.............................................. 49

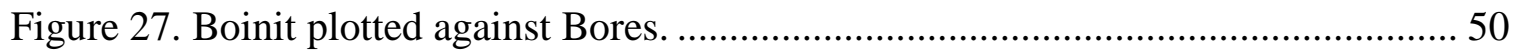

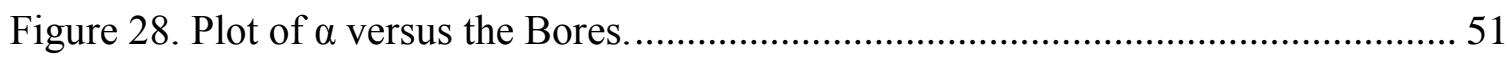

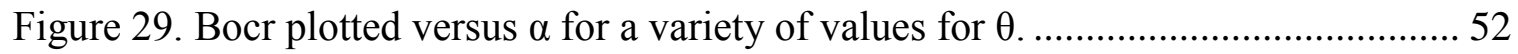

Figure 30. Point-on profile comparison of contact angles on either side of the $\theta=30^{\circ}$

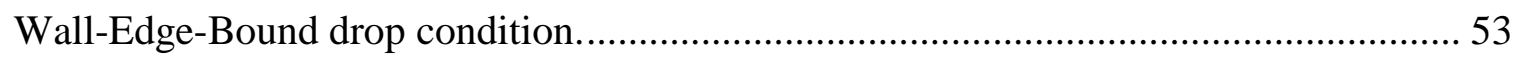

Figure 31. Blade vertex half-angle $\alpha$ plotted against Bocr for $\theta=25^{\circ}$ and $\theta=35^{\circ} \ldots \ldots . .54$

Figure 32. Sketch of a pendant drop with experiment notation employed ..................... 55

Figure 33. Bocr data from Figure 29 after the initial correlation model is applied .......... 56

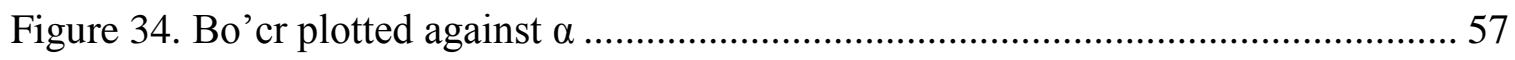

Figure 35. Bottom view of SE-FIT simulations showing dry tips ............................... 58 


\section{Chapter 1}

\section{Introduction}

Experiment time onboard the International Space Station (ISS) is a precious commodity for fluids research. The residual acceleration levels present throughout the ISS are typically on the order of $\mathrm{g}=10^{-6} \mathrm{~g}_{0}$, where $\mathrm{g}_{0}$ is the magnitude of normal Earth gravity. The 'microgravity' condition means that the buoyancy forces are small indeed. The ratio of buoyancy to surface tension forces is characterized by the dimensionless Bond number Bo $\equiv \rho \boldsymbol{a} L^{2} / \sigma$, where $\rho$ is the density difference across an interface, $\boldsymbol{a}$ is the acceleration associated with the body force, $L$ is a characteristic length of the surface, and $\sigma$ is the surface tension. When Bo $<<1$, surface tension forces dominate fluid systems even when the characteristic length of that system is large. In this research, three investigations are pursued where the unique interplay between surface tension forces, wetting characteristics, and system geometry control the fluid behavior whether in large systems aboard spacecraft, or within micro-scale systems on Earth $[1,2]$.

\subsection{Capillary Flow Experiments}

In July 2010, during Expedition 24 aboard the International Space Station (ISS), astronaut Tracy Caldwell-Dyson performed the first operation on one of many handheld vessels included in the second round of Capillary Flow Experiments (CFE-2) [3]. The first series of experiments, CFE-1 [4], concluded in December 2007 and included six vessels, four of which were re-flown for CFE-2 after several alterations [4]. Those four vessels, comprised of two Interior Corner Flow experiments (ICF1, ICF2) and two 
critical Vane Gap wetting (VG1, VG2) experiments are currently in orbit with 30 operations performed to date. An annotated list of the test operations performed by the respective astronauts is provided in Appendix A. Two more vessels, ICF4 and ICF9, arrived on the ISS early this October as part of the SpaceX CRS (Commercial Resupply Service) mission [5], and another 5 vessels are expected to be launched to the ISS shortly. The CFE experiments have two primary thrusts: They pursue fundamental science to confirm current theory, as well as provide application specific results of use to spacecraft systems design.

Prior to launch, we provide astronaut training concerning the basic motivation behind the CFE experiments as well as the basic experiment operations they will use to conduct the procedures. Simple yet detailed step-by-step procedures are written to assist the ISS crew members. Due to gaps in satellite coverage, the ISS experiences several Loss of Signal (LOS) periods during every orbit which can last from several minutes to tens of minutes. During these periods especially, the crew procedures are vital to the astronauts so that scientific data is not lost.

In the spring of 2011 a NASA ground station was constructed at Portland State University to support both the CFE and Capillary Channel Flow (CCF) experiments [6,7]. The ground station, shown in Figure 1, includes two computer terminals with 5 monitors and 2 LED TV monitors, two uninterruptable power supplies, multiple redundant data backup systems, and headsets for two-way communication over the NASA Voice over Internet Protocol (VoIP) loops. In January 2012, at the request of astronaut Don Pettit, PSU was first enabled for direct communication with ISS crew members operating CFE 
experiments. Astronaut feedback combined with a preexisting PSU connection to live footage from both internal and external ISS cameras allows for real time adjustments in experiment procedures and test points so that a higher quantity of data can be collected with greater resolution.

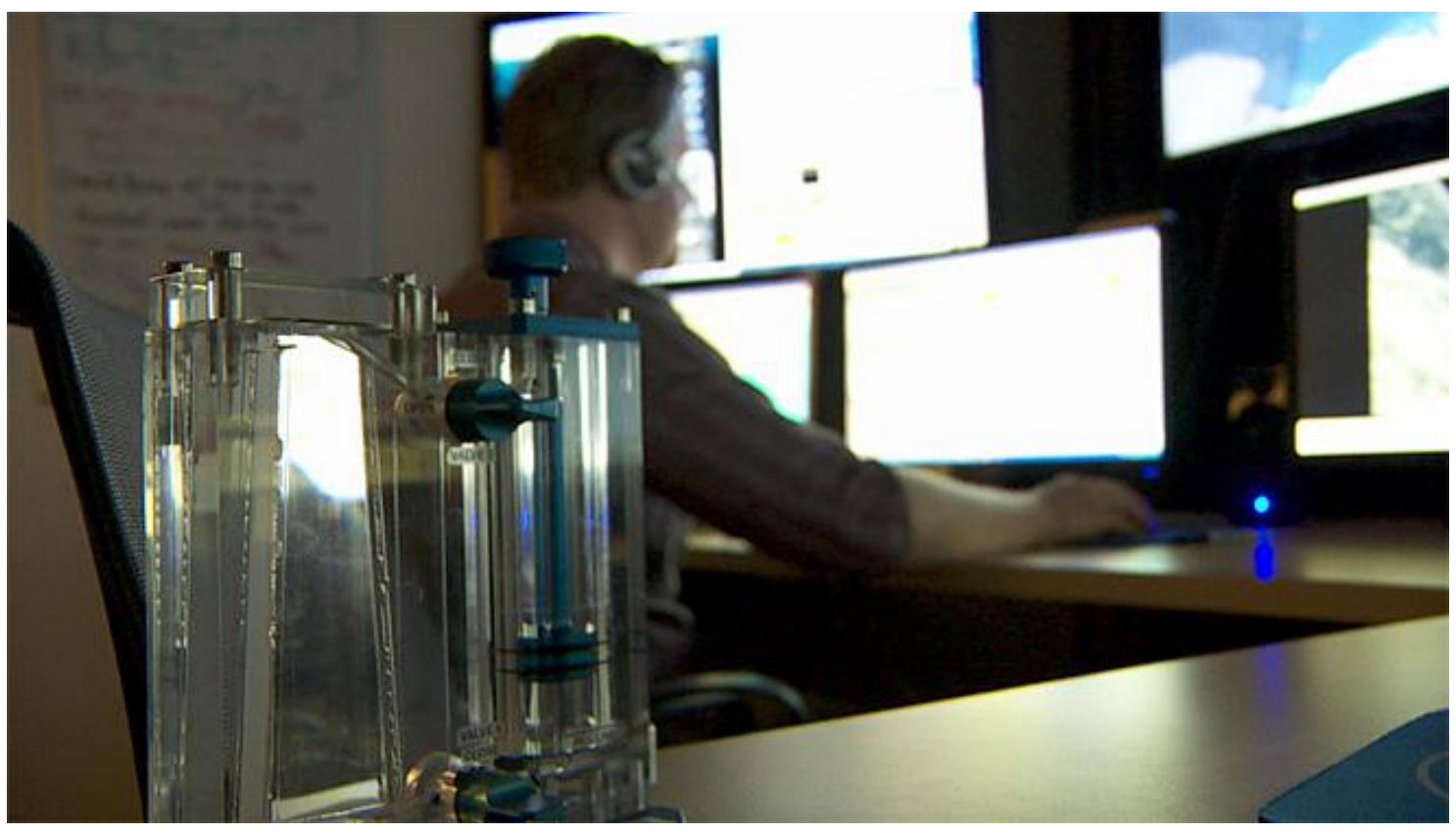

Figure 1. Image of the PSU NASA ground station with the ICF1 engineering model in the foreground. Software updates and security checks are regularly required to maintain access to NASA's secure network.

Since the creation of the PSU ground station, 15 CFE operations have been supported, with 12 including direct communication with astronauts Don Pettit, Joseph Acaba, or Station Commander Dan Burbank. One CCF ‘experiment run’ was also supported amounting to 27 days of continuous $24-7$ operation.

Data acquired to date from VG vessels are offered in Chapter 2. Additionally, a new graphical method for interpreting VG critical angle and bulk shift data is presented which allows for quicker, and perhaps more intuitive interpretation of the findings. 


\subsection{Compressed Bubble Migration}

As a result of a large number of experiments conducted aboard the ISS, significant unplanned discoveries are becoming more commonplace. Such 'science of opportunity', a phrase coined by astronaut Don Pettit, often takes place on the periphery of other experiments prompting further, though perhaps tangential, investigations. Recently, observations during the operation of CFE-2 experiments ICF1 and ICF2 have identified an unexplained phenomenon in the form of migrating air bubbles following a rapid compression. The air bubbles are observed to drift along paths with vector components common to the residual acceleration. This is expected due to density-driven convective currents. However, the bubble velocities measured are up to three orders of magnitude higher than those attributable to buoyancy in the appropriate Stokes flow limit [8]. Likely mechanisms are explored analytically and experimentally while citing prior theoretical works that may have anticipated such phenomena. Once properly understood, compressed bubble migration may be used as an elegant method for phase separation in spacecraft systems or microgravity-based materials manufacturing. A description of the incidental observations, purposeful experiments, data reduction efforts, and proposed explanations are the focus of Chapter 3.

\subsection{Wall-Edge-Vertex-Bound Drops}

Drops bound to solid surfaces, edges, and vertexes play a major role in both nature and industry. In low gravity environments liquid drops or bubbles preferentially become bound to surfaces as opposed to floating free in the medium. In May 2011 this became a 
problem when an Extra Vehicular Activity (EVA) to install new experiments and fixtures to the exterior of the ISS was cut short due to a faulty sensor [9]. It is speculated that a fluid droplet had become bound to the surface of the sensor which caused its malfunction. The surface energy of such a wall-bound drop is at a local minimum, requiring some form of perturbation to dislodge it from the surface. Knowledge of the perturbations required to dislodge such drops provides a valuable design guide.

One such perturbation is provided by gravity. Expanding on research performed by Chen et al. [10], which focused on drops bound by the edge of 2-D planes of infinite length, so called 'wall-edge-vertex bound drops' are explored using a numerical approach which applies the Surface Evolver algorithm [11] through implementation of a new file layer and a multi-parameter sweep function. As part of a team of five students in a graduate studies course, thousands of critical drop configurations are efficiently computed as functions of contact angle, blade edge vertex half-angle, and g-orientation in the plane of the vertex.

Utilizing SE-FIT [12], a graphical user interface developed at Portland State University for K.A. Brakke's Surface Evolver [11], the numerical simulations were conducted to determine critical Bond numbers $\mathrm{Bo}_{\mathrm{cr}}$ at which Wall-Edge-Vertex-Bound drops will detach from surfaces. In Chapter 4 these results are benchmarked against previous numerical simulations and quantitative comparisons are made against laboratory experiments. It is shown that sessile, pendant, and wall-edge bound drops are only limiting cases of the more generalized blade-bound drops, and that an intriguing 'dry leaf tip' is observed for a range of the critical geometric and wetting parameters. 
The behavior of drops on such surfaces is key to practical applications ranging from spray pesticides to condensing heat exchangers. Particularly interesting applications arise in reduced acceleration environments such as those common aboard orbiting spacecraft. In such instances drops and bubbles can possess volumes $10^{6}$-fold larger than their normal-gravity counterparts. Knowledge of the stability limits of such drops may be critical to the performance of numerous systems aboard spacecraft, particularly as regards liquid fuels, cryogens, phase change thermal control systems, and water processing systems from life support [13]. 


\section{Chapter 2}

\section{Capillary Flow Experiments}

\subsection{Background}

The second round of Capillary Flow Experiments (CFE-2) for the International Space Station (ISS) was introduced in [4]. In that paper the experiment and design plan were described. Since then, six of the eleven handheld experiment vessels have been launched to ISS and a description of the experiments and ongoing data reduction efforts is the focus of this Chapter. However, a brief overview of the experiment is unavoidable and is provided here for completeness, largely drawing upon from prose continued in [3].

Capillary flows continue to be critical to myriad fluids management systems in lowgravity environments: fuels/cryogen storage systems, thermal control systems (e.g., vapor/liquid separation), life support systems (e.g., water recycling), and materials processing in the liquid state. Under microgravity conditions, capillary forces can be exploited to control fluid orientation so that such large mission-critical systems perform predictably. The second Capillary Flow Experiment (CFE-2) is a study that can yield quantitative results from safe, low-cost, short time-to-flight, handheld fluids experiments. The experiments aim to provide results of interest and value to the capillary flow engineering community that cannot be readily achieved in ground-based tests. CFE-2 derives from the first CFE study (CFE-1) which is discussed in a comprehensive report [4]. 


\subsection{CFE-2 Experiments}

CFE-2 consists of handheld vessels that investigate interior corner flows (ICF vessels) and critical vane gap wetting (VG vessels). My primary contribution to CFE-2, ongoing data reduction efforts for the Vane Gap experiments, are detailed herein with addtitional contributions to the ICF experiments to be found in Appendix B. A chronology of the first thirty flight experiments is listed in Appendix A along with the particular astronaut experimenters noted.

\subsubsection{The Vane Gap Experiments (2 units VG1 and VG2)}

The second CFE-2 experiment type are the Vane Gap experiments. These experiments seek to identify a fundamental wetting condition akin to the critical corner wetting condition analyzed by Concus and Finn [14], but for interior corners formed by walls that possess a gap at the virtual axis of intersection of the two walls. Such a 'wallvane gap' is common in spacecraft systems, but should not be cavalierly treated as an ideal corner. The original CFE tests were highly successful uncovering the complex interaction of geometry, contact angle, asymmetry, and gap wetting; both statics and dynamics. The most detailed review of the work to date is contained in Chen et al. [15]. The current VG experiments aim to determine critical wetting conditions for perforated sheets for perfectly wetting fluids. Such constructs are used in tankage systems (e.g. Skinner et al. [16] and papers by Jaekle [17]) and serve as models for screens and perforated sheets, plates, vanes, etc. The impact of such 'porous substrates' is expected to be significant; with immediate implications to the design of passive geometries to manage highly wetting fuels, cryogens, thermal fluids, and contaminated aqueous 
solutions (i.e. urine processors).

Two perforation-types are employed to test two edge conditions. A second objective is to determine critical wetting transients in such cases while a third is to validate numerical predictions and establish a solution protocol for the disparate length scales and discontinuous, or nearly discontinuous, wetting phenomena. Vane Gap schematics are depicted in Figure 2 with container details listed below:

VG1 and VG2 (ref. Figure 2)

1. Ellipse Section:

Major $/$ minor axis $5.08 \mathrm{~cm} / 3.386 \mathrm{~cm}$, Height is $12.7 \mathrm{~cm}$

2. Vane dimensions:

$3.30 \mathrm{~cm}$ by $0.238 \mathrm{~cm}$ by $11.43 \mathrm{~cm}$, square corner centered pattern VG1—perforation dimensions: $3 \mathrm{~mm}$ ID on $4.4 \mathrm{~mm}$ centers VG2 - perforation dimensions: $2 \mathrm{~mm}$ ID on $4.4 \mathrm{~mm}$ centers

3. Vane pivot axis coaxial with ellipse

4. Gap minimum distance is $0.4318 \mathrm{~mm}$ when aligned with minor diameter

5. Vane angle rotation $360^{\circ}$ with $0.5^{\circ}$ resolution

6. Fluid is $10 \mathrm{cs}$ Si oil

7. Initial fluid fill volume is $51 \mathrm{ml}$

In a strict sense, the critical corner wetting condition [14] is radically altered for interior corners that do not actually contact. The VG experiments test a specific selection of vane-wall types. Vane gap and vane-wall angle is varied by repeatedly sweeping about both sub- and super-critical wetting regimes as well as the wetting hysteresis map. The 
container consists of an elliptic cylindrical test vessel with an axial vane that can be pivoted changing both the angle of the vane and wall and the size of the vane-wall gap as depicted in Figure 2. Two perforated vanes are employed with varying open areas. Each vane employs one smooth and one serrated edge so that two gap-types can be tested for each container.

After injecting the prescribed amount of fluid the crew member rotates the vane at intervals in part determined by the astronaut allowing significant time (up to 15 minutes) for the fluid to equilibrate between each interval. At a critical vane angle the fluid will spontaneously wet along the vane edge at which point the vane angle will be measured for comparison to theory. The pinning edges provided by the perforations contribute to an altered effective (in this case, nonzero) contact angle $[18,19,20]$. The serrated vane edge contributes to an altered effective (in this case, larger) vane gap distance.
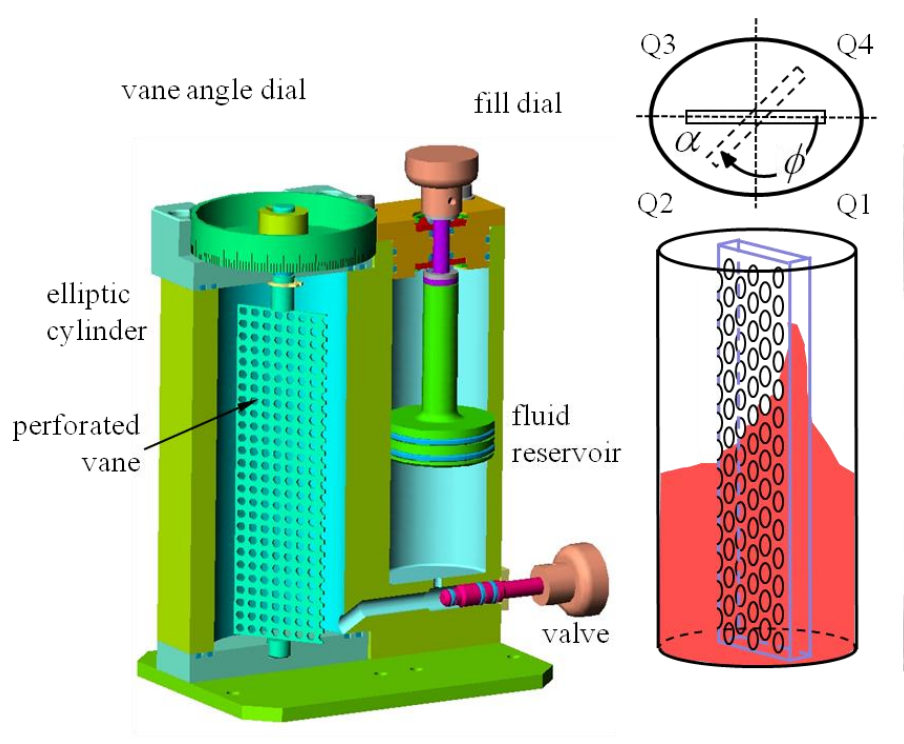

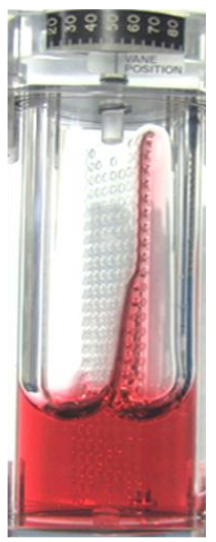

CFE-2 VG1

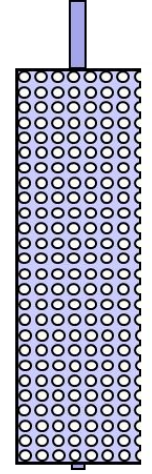

VG1

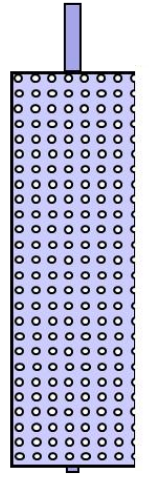

VG2

Figure 2. From left to right: Solid model of VG1, schematic of critical wetting with vane rotation, an image taken during CFE-2 VG1 operations on ISS (smooth gap wetting), and perforated vanes of VG1 and VG2. Note that $\varphi$ represents the location of the smooth gap relative to the major axis.

Twenty operations have been performed to date on the VG vessels by six astronauts. 
Primary planned science operations have been completed for the VG1 vessel which includes: Two operations in the clockwise $(\mathrm{CW})$ direction, one in the counter-clockwise (CCW) direction, two targeting specific critical angles in quadrants Q1/Q2 and Q3/Q4, and six similar operations repeated on the vessel with the vane perforations filled with fluid. Nine operations have been performed to date on VG2, nearly completing the primary science objectives, with the exception of quadrant specific tests. Current critical angle ranges are available for VG1 and VG2 in Table 1 and Table 2 respectively, with visual representation of select data sets in Figure 3 and Figure 5.

Table 1. Current data of all 16 VG1 critical angles with unfilled perforations, and 16 with filled perforations. The approximate range within which the true 'critical' value resides is shown. Repeatability and accuracy is as high as $\pm 0.25^{\circ}$. Angles $\varphi$ and $\alpha$ are shown in degrees.

\begin{tabular}{|c|c|c|c|c|c|}
\hline \multirow{2}{*}{ Critical Point } & \multirow[b]{2}{*}{ Quadrant } & \multicolumn{2}{|c|}{ VG1 - Unfilled } & \multicolumn{2}{|c|}{ VG1 - Filled } \\
\hline & & $\varphi$ & $\alpha$ & $\varphi$ & $\alpha$ \\
\hline \multirow[t]{4}{*}{$\underline{\text { Smooth Gap Wetting }}$} & Q1 & $50.5-51$ & $50.5-51$ & $42.5-43.5$ & $42.5-43.5$ \\
\hline & Q2 & $129.5-130$ & $50-50.5$ & $135-136$ & $44-45$ \\
\hline & Q3 & $233-234$ & $53-54$ & $226.5-227$ & $46.5-47$ \\
\hline & Q4 & $305.5-306.5$ & $53.5-54.5$ & $311-312$ & $48-49$ \\
\hline \multirow[t]{4}{*}{ Serrated Gap Wetting } & Q1 & 63-64 & $63-64$ & $60-61$ & $60-61$ \\
\hline & Q2 & $117-117.5$ & $62.5-63$ & $122-124$ & $56-58$ \\
\hline & Q3 & $246.5-250$ & $66.5-70$ & $246-247$ & $66-67$ \\
\hline & Q4 & $294-295.5$ & $64.5-66$ & $296-297$ & $63-64$ \\
\hline \multirow[t]{4}{*}{$\underline{\text { Smooth Gap De-Wetting }}$} & Q1 & $49.5-50$ & $49.5-50$ & $43.5-48.5$ & $43.5-48.5$ \\
\hline & Q2 & $129.5-134$ & $46-50.5$ & $135.5-137$ & $43-44.5$ \\
\hline & Q3 & $232.5-235.5$ & $52.5-55.5$ & $227-229$ & $47-49$ \\
\hline & Q4 & $306.5-307$ & $53-53.5$ & $311-312$ & $48-49$ \\
\hline \multirow[t]{4}{*}{ Serrated Gap De-WEetting } & Q1 & $46.5-47$ & $46.5-47$ & $43-47$ & $43-47$ \\
\hline & Q2 & $133-135.5$ & $44.5-47$ & $137-138$ & $42-43$ \\
\hline & Q3 & $229.5-230$ & $49.5-50$ & $228.5-229$ & $48.5-49$ \\
\hline & $\mathrm{Q} 4$ & $309.5-312.5$ & $47.5-50.5$ & $313-313.5$ & $46.5-47$ \\
\hline
\end{tabular}

Each operation has served to narrow the range within which the critical angles are believed to be. Detailed observations are taken of the critical angles during and after each 
test so that changes to experiment procedures can be made in advance of the next run. With the newly available direct-to-astronaut communications during experiments, procedures can be amended 'on the fly'. By communicating what the science goals of each operation is, and what to look for, astronauts can identify critical angles and other minute fluid interface changes often before science support teams on the ground. Such detailed feedback allows for greater resolution of the critical angle ranges and quicker test point turn around, thus allowing for more test point to be obtained per operation or longer wait times at specific angles to ensure a deep equilibrium state.

Table 2. Current data of all 16 VG2 critical angles with unfilled perforations, and 16 with filled perforations. The approximate range within which the true 'critical' value resides is shown. Repeatability and accuracy is as high as $\pm 0.25^{\circ}$. Angles $\varphi$ and $\alpha$ are shown in degrees.

\begin{tabular}{|c|c|c|c|c|c|}
\hline \multirow{2}{*}{ Critical Point } & \multirow[b]{2}{*}{ Quadrant } & \multicolumn{2}{|c|}{ VG2 - Unfilled } & \multicolumn{2}{|c|}{ VG2 - Filled } \\
\hline & & $\varphi$ & $\alpha$ & $\varphi$ & $\alpha$ \\
\hline \multirow[t]{4}{*}{$\underline{\text { Smooth Gap Wetting }}$} & Q1 & $48.5-49$ & $48.5-49$ & $44-45.5$ & $44-45.5$ \\
\hline & Q2 & $130-132$ & $48-50$ & $135.5-140$ & $40-44.5$ \\
\hline & Q3 & $228-228.5$ & $48-48.5$ & $226-228$ & $46-48$ \\
\hline & Q4 & $311-312$ & $48-49$ & $315-320$ & $40-45$ \\
\hline \multirow[t]{4}{*}{ Serrated Gap Wetting } & Q1 & $59-60$ & $59-60$ & $54-55$ & $54-55$ \\
\hline & Q2 & $123-126$ & $54-57$ & $126.5-128$ & $52-53.5$ \\
\hline & Q3 & $235-236$ & $55-56$ & $232-235$ & $52-55$ \\
\hline & Q4 & 304-306 & $54-56$ & $305-307$ & $53-55$ \\
\hline \multirow[t]{4}{*}{$\underline{\text { Smooth Gap De-Wetting }}$} & Q1 & $46-47$ & $46-47$ & $43.5-44$ & $43.5-44$ \\
\hline & Q2 & $133.5-134$ & $46-46.5$ & $133.5-135$ & $45-46.5$ \\
\hline & Q3 & $225-226$ & $45-46$ & $225.5-228$ & $45.5-48$ \\
\hline & Q4 & $314-315.5$ & $44.5-46$ & $316-318$ & $42-44$ \\
\hline \multirow[t]{4}{*}{ Serrated Gap De-WEetting } & Q1 & $48.5-49$ & $48.5-49$ & $45.5-48$ & $45.5-48$ \\
\hline & Q2 & $133-134$ & $46-47$ & $134.5-136$ & $44-45.5$ \\
\hline & Q3 & $227-227.5$ & $47-47.5$ & $224-227$ & $44-47$ \\
\hline & Q4 & $313.5-314$ & $46-46.5$ & $315-320$ & $40-45$ \\
\hline
\end{tabular}

Observation of the critical angle $\alpha$ sheds light on the apparent asymmetries of the test chamber, which are known to $\pm 20 \mu \mathrm{m}$ from pre-flight inspection of the hardware. 
Additional tests are currently planned for VG2 to further refine all critical angles to $< \pm 0.5^{\circ}$

Another significant avenue of research stemming from the VG vessels, first identified in CFE-1, is a previously unexpected 'bulk shift' phenomenon. Minute differences in the mean curvature of the bulk menisci due to miniscule asymmetries in the container predisposed the VG vessels to weak capillary pressure gradients. Fluid then flows from one side of the vane to the other [21] in a process referred to as a bulk shift (BS) flow (seen in Figure 4), and Reverse Bulk Shift (RBS) when the flow acts to equalize the fluid levels.
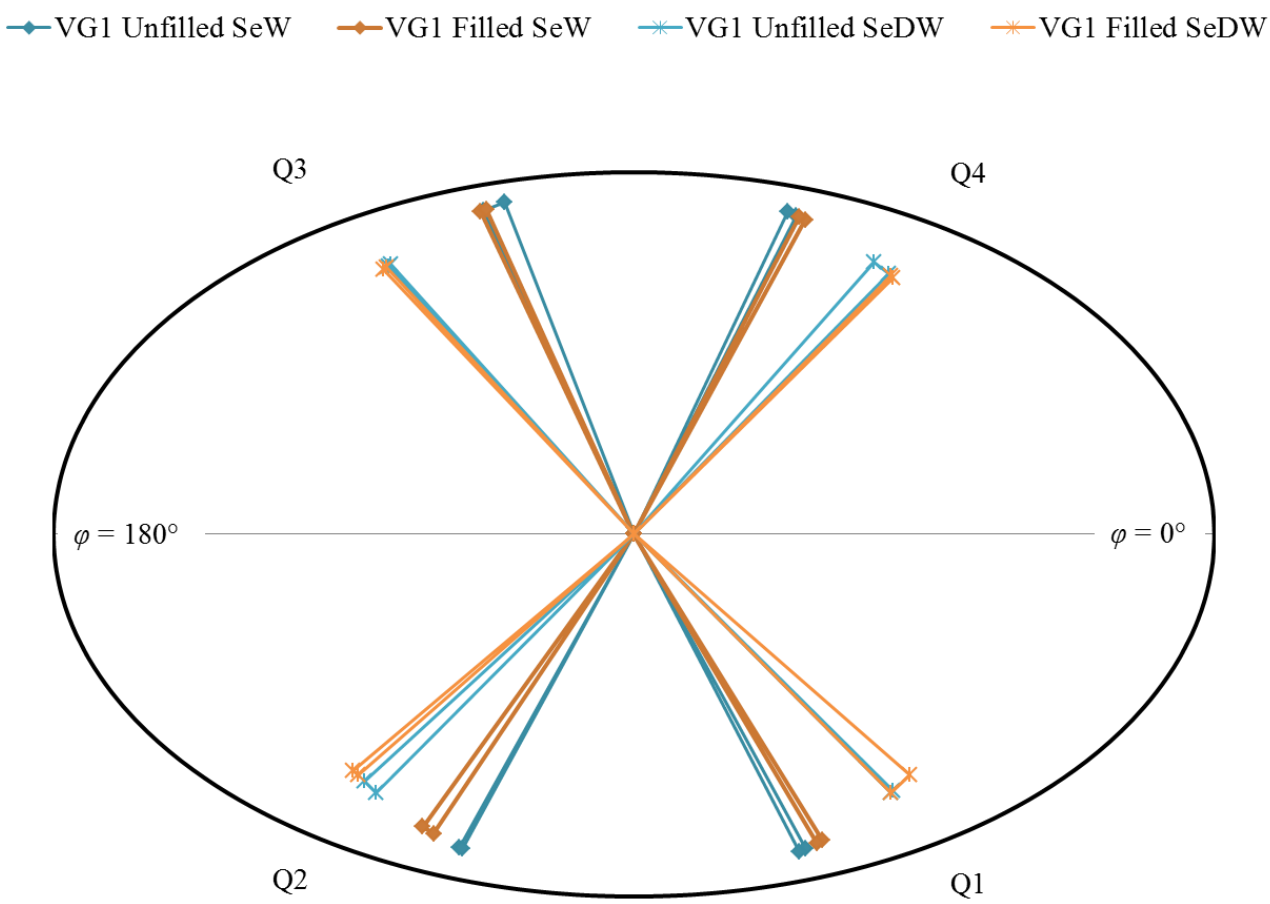

Figure 3. Top down view of the VG1 vessel comparing the critical angles and ranges of Serrated Gap Wetting (SeW) and De-Wetting (SeDW) in the filled and unfilled perforation configurations. Line lengths from the origin to the data points are scaled to container dimensions to show the vane edge relative to the wall at the critical angles. Filled perforations appear to have little effect on serrated gap critical angles, though some shift is apparent in Q1 and Q2. 


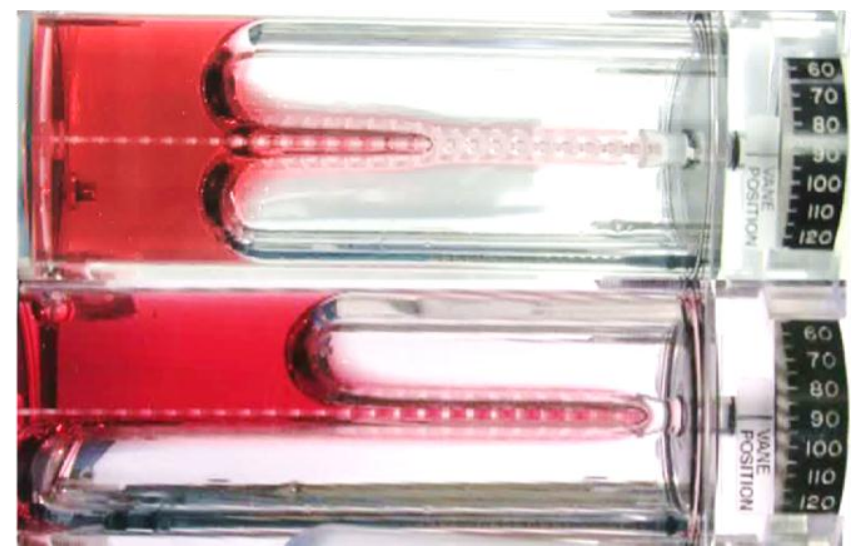

Figure 4. Comparison of VG1 vessel at $\varphi=90^{\circ}$ with empty perforations (above) and filled perforations (below). Understanding Bulk Shift phenomenon is important to avoid potentially catastrophic fluid reorientation in vulnerable spacecraft systems such as fuel tanks.

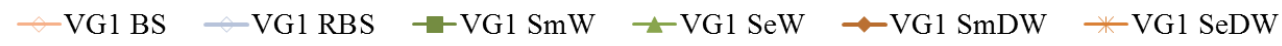

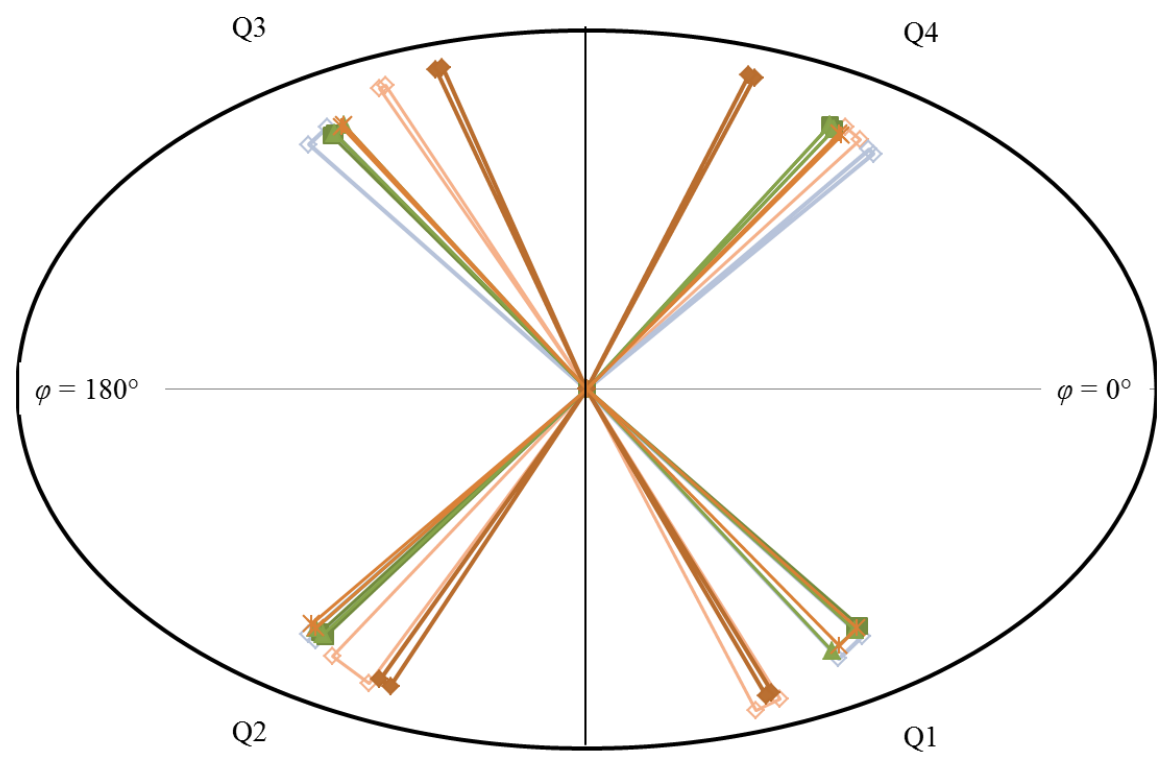

Figure 5. A top down view of the VG1 vessel in the filled perforation configuration with critical angles and ranges shown for Bulk Shift (BS), Reverse Bulk Shift (RBS), Smooth Gap Wetting (SmW) and De-Wetting (SmDW), and Serrated Gap Wetting/De-Wetting.

A detailed analysis of bulk shift mechanisms can be found in [21], but is outside of the scope of this thesis. In brief, the bulk shift seen in Figure 4 is referred to as a disconnected bulk shift (DBS) because either side of the vane is connected by secondary 
flows along the gaps. Conversely, a connected bulk shift (CBS) occurs with no gap flows.

Figure 5 shows a CBS in Q4 for the VG1 filled perforation configuration.

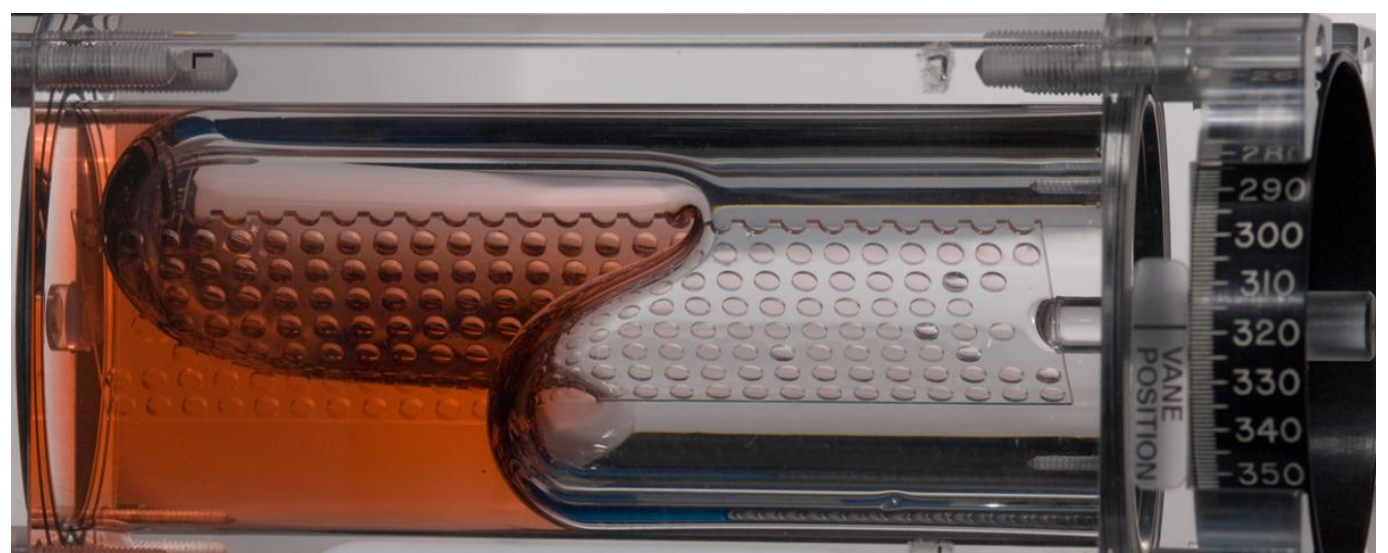

Figure 6. An image taken by astronaut Don Pettit during VG1-16 test operations. No gap flows are present at this angle, yet preferential reorientation of the fluid to one side of the container is observed. Such a configuration is referred to as a connected bulk shift (CBS).

Bulk shifts can be prevented by the presence of unfilled perforations (see Figure 4). Unfilled perforations increase the effective contact angle of the vane surface and resist the bulk shift configuration. Several operations of both VG vessels are planned to examine specific filled/unfilled perforation conditions which may result in a bulk shift, or the lack thereof.

An effective contact angle for a vane with perforations can be calculated by

$$
\operatorname{Cos}\left(\theta_{\text {eff }}\right)=\left[A_{\text {open }} \operatorname{Cos}\left(\theta_{\text {open }}\right)+A_{\text {vane }} \operatorname{Cos}\left(\theta_{\text {vane }}\right)\right] / A_{\text {tot }},
$$

where $\theta_{\text {eff }}, \theta_{\text {open, }}$ and $\theta_{\text {perf }}$ are the effective vane contact angle, perforation contact angle, and solid vane contact angles, respectively; and $A_{\text {open, }} A_{\text {vane, }}$ and $A_{\text {tot }}$ are area of the unfilled perforations, solid vane, and total vane area, respectively. By assuming that $\theta_{\mathrm{open}}=90^{\circ}$ for unfilled perforations and $\theta_{\text {vane }}=0^{\circ}$ for the solid vane Equation 1.1 becomes

$$
\operatorname{Cos}\left(\theta_{\text {eff }}\right)=\frac{A_{\text {vane }}}{A_{\text {tot }}}=(1-\% \text { perf }) \operatorname{Cos}\left(\theta_{\text {vane }}\right)
$$


where \%perf refers to the percentage of filled versus unfilled perforation area. Table 3 shows effective contact angles for both VG vessels should $0,1,3,5$, or 7 perforation columns be left unfilled.

Table 3. Effective contact angles (in degrees) for both VG vessels with varying number of perforation columns left unfilled.

\begin{tabular}{cccccc} 
Vessel & 0 Columns & 1 Columns & 3 Columns & 5 Columns & 7 Columns \\
\hline VG1 & 48.8 & 45.0 & 36.4 & 25.5 & 0 \\
VG2 & 32.0 & 29.5 & 24.0 & 16.9 & 0
\end{tabular}

Complete tables of the current ranges of the bulk shift angles for both VG1 and VG2 are available in Appendix B.

\subsection{Directions}

The crew procedures for each run of CFE-2 on ISS are written to require between 3 to 4 hours for set-up, operations, and tear-down. Downlink of the HD video normally occurs on the following day. This relatively short period allows the 'opportunistic' experiments to be conducted on an irregular or short schedule when crew time is available. Approximately 5 additional runs are planned for the VG test units currently onboard ISS with another 12 planned for the newly arrived ICF4 and ICF9 vessels. There is currently no plan to return the flight units to Earth and the 5 remaining units will be shipped to ISS by spring of 2013. In the meantime, large amounts of data continue to be collected for ICF1, ICF2, VG1, and VG2. The data will be further reduced for exhaustive comparisons with theory and numerics. Application of the results will continue to expand as solutions are confirmed and published. 


\section{Chapter 3}

\section{Compressed Bubble Migration}

\subsection{Background}

The second Capillary Flow Experiment (CFE-2) is a series of handheld fluids experiments currently under investigation on the ISS. The primary science objectives concern capillary flows in complex containers, but incidental observations of interest have led to potentially valuable 'extra science' observations and experiments. A status of the CFE-2 experiment was overviewed in Chapter 1 and detailed Appendix B. Recently, peripheral observations have identified an unexplained phenomenon in the form of migrating bubbles following a rapid compression. A description of these incidental observations, purposeful experiments, preliminary data reduction efforts, and proposed explanations are the focus of this Chapter.

\subsection{CFE-2 Experiments and Bubble Migration Observation}

CFE-2 consists of tissue box-sized vessels that investigate critical geometric wetting conditions and capillary flows in complex containers. The containers constructed concerning the latter are called Interior Corner Flow (ICF) vessels. The ICF experiments seek to determine the rates of 3-D imbibition of wetting liquids in containers of systematically increasing complexity, the dependence of critical model dynamical boundary conditions as a function of geometry, and the performance of such devices as passive phase separators (i.e., converting bubbly flows to separated continuous gas and liquid inventories). 
There are two ICF container types. A solid model perspective is provided in Figure 7 for both ICF1 and ICF2. The primary components are a piston operated reservoir and an initially gas-filled test chamber. The general procedure for the experiment is to open Valve 1, quickly dispense the liquid into the test chamber by hand by turning the piston knob, and then simply video recording the liquid flow from the base of the container to the vertex. The passive capillarity-driven flow in the test chamber is considered the 'primary science.'

As identified in Figure 7, the ICF vessels employ a transport tube to pass liquid from the reservoir to the test chamber, but also to return the liquid from the vertex of the test chamber to the reservoir for repeat runs. The transport tube may be sealed off from the test chamber by the two 1/4-turn valves (Valve 1 and Valve 2). On occasion, a bubble becomes trapped in the transport tube and is subsequently compressed as the total volume of the system is reduced during the liquid deployment. It was observed that the accidentally trapped bubble moves following the compression. As a curiosity on the periphery of the primary science experiments, we tracked the bubble path and quickly found significantly higher velocities than explicable by background acceleration levels alone. On three occasions we observed the behavior inadvertently after which we sought to devote two subsequent experiment opportunities (3hrs each on the ISS) in an attempt to recreate the phenomenon purposefully. A chronology of the CFE-2 experiments where the migration events occurred is listed in Table 4. Relevant properties of the ICF1 and ICF2 containers and fluid properties are listed in Table 5. 


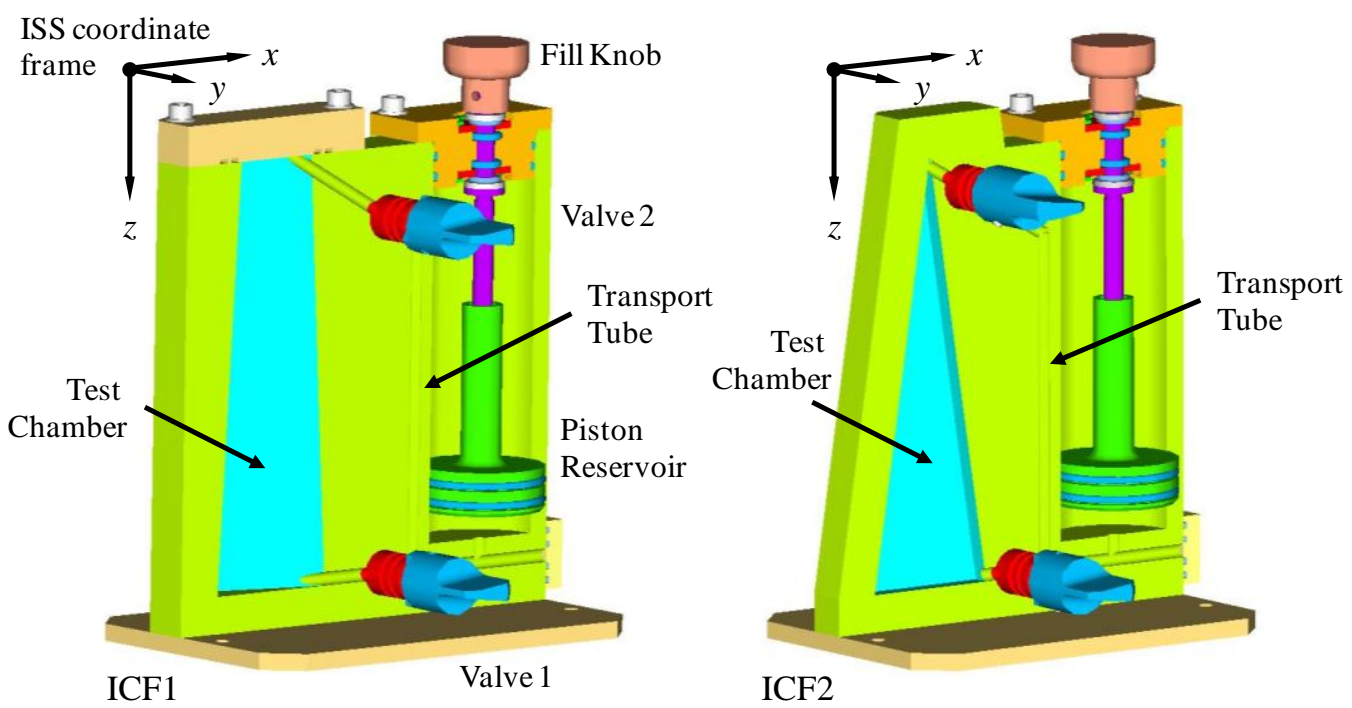

Figure 7. Solid perspective models of ICF1 and ICF2 vessels with primary components labeled. The ISS reference frame is noted as are the transport tubes.

Table 4. CFE-2 compressed bubble migration observation chronology.

\begin{tabular}{|c|c|c|c|}
\hline $\begin{array}{l}\text { Test } \\
\text { Cell }\end{array}$ & GMT & Astronaut & Note \\
\hline ICF1-3 & $\begin{array}{l}\text { 2010-197: } \\
\text { 10:00-11:35 }\end{array}$ & $\begin{array}{l}\text { T. Caldwell- } \\
\text { Dyson }\end{array}$ & $\begin{array}{l}\text { Peripheral observation: Bubble compression occurs over a } \\
1 \text { min duration and results in a bubble migration lasting } \\
\text { approximately } 10 \mathrm{~min} \text {. }\end{array}$ \\
\hline ICF1-4 & $\begin{array}{l}\text { 2010-252: } \\
08: 15-09: 50\end{array}$ & S. Walker & $\begin{array}{l}\text { Peripheral observation: Bubble compression occurs out of } \\
\text { view of the camera. However, the bubble motion becomes } \\
\text { visible for } 29 \mathrm{~s} \text {. }\end{array}$ \\
\hline ICF2-3 & $\begin{array}{l}\text { 2010-302: } \\
14: 15-15: 45\end{array}$ & S. Kelly & $\begin{array}{l}\text { Peripheral observation: The compression occurs over a } 60 \mathrm{~s} \\
\text { period and } 70 \mathrm{~s} \text { of motion is observed before the bubble leaves } \\
\text { the field of view. }\end{array}$ \\
\hline ICF2-6 & $\begin{array}{l}\text { 2011-123: } \\
\text { 11:30-13:00 }\end{array}$ & R. Garan & $\begin{array}{l}\text { Bubble compression-migration experiment performed } \\
\text { purposefully as a new science investigation. The compression } \\
\text { occurs over a } 30 \text { s period and bubble motion is observed for } \\
4 \text { min before coming to rest. During this test the bubble } \\
\text { diameter decreases with time and after approximately } 15 \mathrm{~min} \text {, } \\
\text { dissolves completely into the liquid. }\end{array}$ \\
\hline ICF1-8 & $\begin{array}{l}\text { 2012-66: } \\
\text { 17:00-19:00 }\end{array}$ & D. Burbank & $\begin{array}{l}\text { Second purposeful bubble compression-migration experiment. } \\
\text { A decompression occurs over } 20 \text { s and bubble motion is } \\
\text { observed for } 18 \mathrm{~min} \text {. During this test the bubble diameter } \\
\text { increases with time, while, strangely, a slug further along the } \\
\text { transport tube decreases with time. }\end{array}$ \\
\hline
\end{tabular}




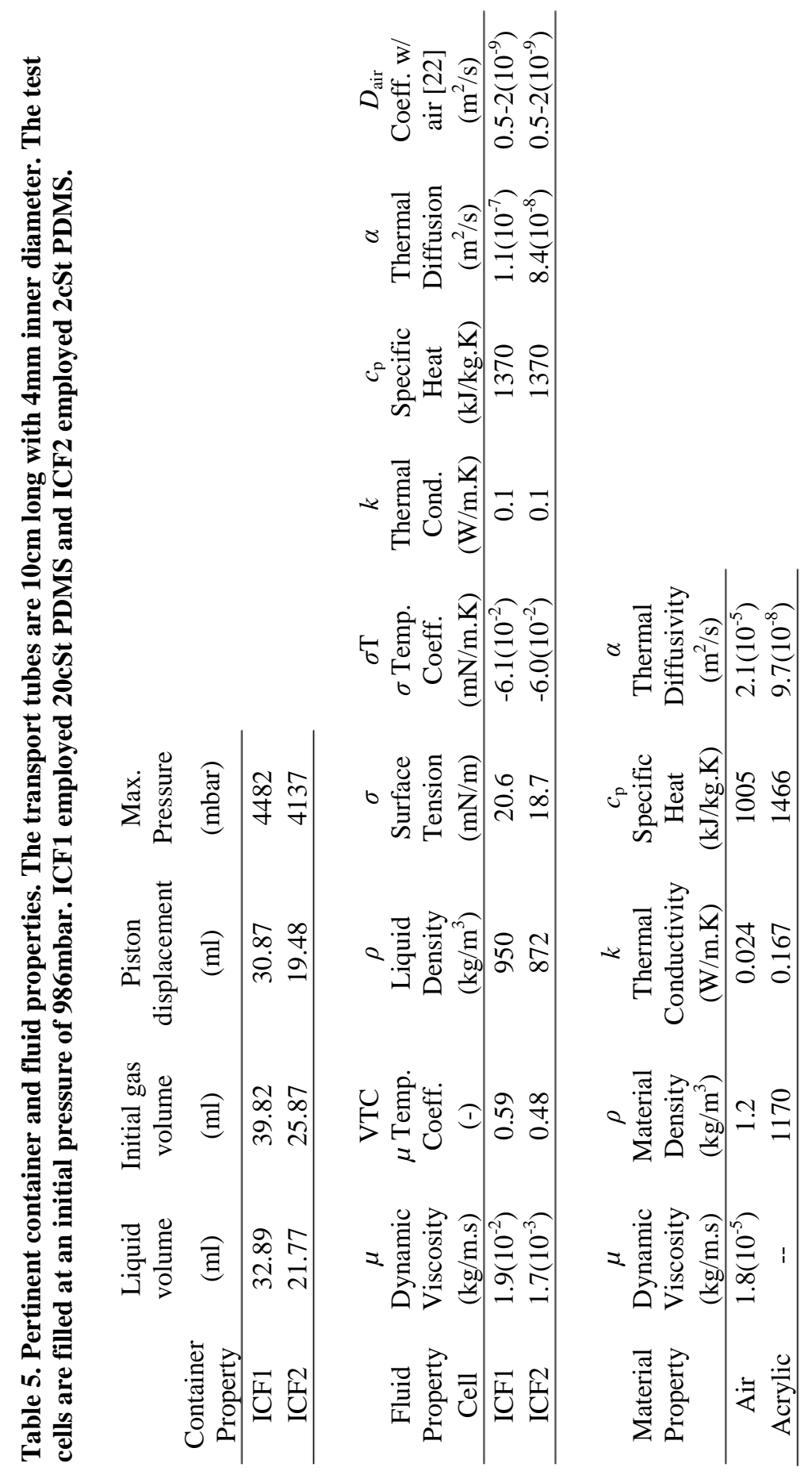




\subsection{Compressed Bubble Migration Data}

During the primary science ICF operations bubbles are generated in the vessels through air ingestion during withdrawal of fluid from the test chamber, or by oscillatory perturbations to the vessel to intentionally create bubbles for phase separation tests. Nearly all of these bubbles eventually coarsen and rejoin the bulk gas in the test chamber, but bubbles entrained in the transport tube remain there. It was such accidentally trapped bubbles that were first observed to move after subsequent rapid compressions.

Accidental observations of trapped bubbles prompted purposeful attempts to recreate this phenomenon. Unfortunately, manipulating multiphase fluid systems in low-g environments can be an involved process. One complication is that gas bubbles that remain in a slug configuration in the transport tube after compression do not move. Such a stable slug is identified in Figure 8. Therefore, an intentionally trapped bubble must have an initial volume such that compression results in a diameter less than that of the $4 \mathrm{~mm}$ transport tube. Thus, to optimize bubble diameters for image analysis, a gaseous slug must be created at the right location in the transport tube to be compressed to a spherical bubble of a desired radius.

One method improvised in-flight to generate an appropriate bubble size is established by exploiting the ICF vessels' corner wetting conditions and the patience of astronaut R. Garan. A small amount of fluid is deployed into the test chamber which wicks along the interior edges, collecting at the apex. With Valve 1 closed and Valve 2 open, liquid is withdrawn by the piston into the transport tube until a gas slug of desired length is ingested into the tube. The liquid then backfills the transport tube by continued wicking 
along the interior corners of the container toward the apex. The gas slug is then

positioned in the center of the tube by iterative adjustment of the valves and piston.
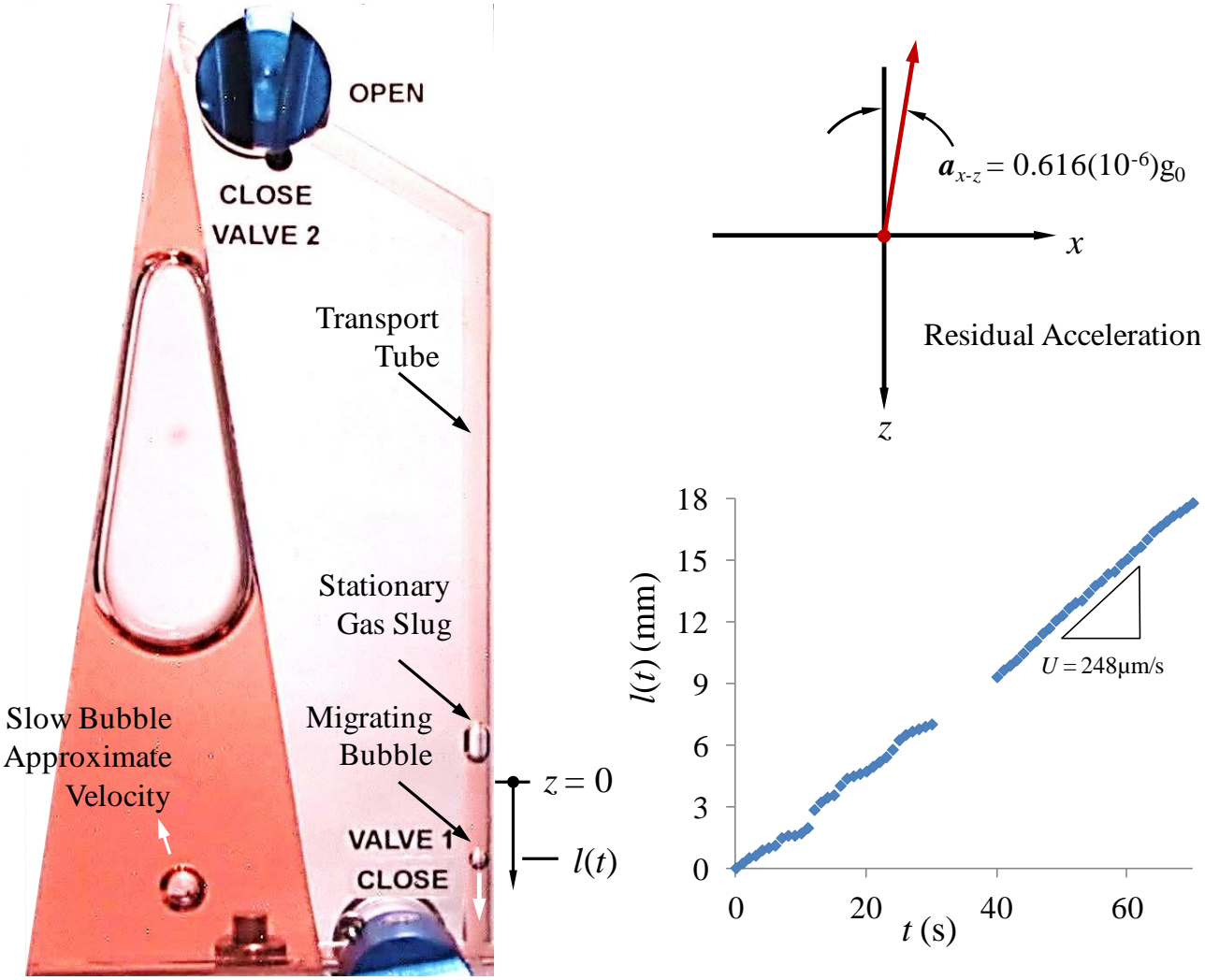

Figure 8. ICF2-3 with a $2.89 \mathrm{~mm}$ diameter bubble traveling downward at $248 \mu \mathrm{m} / \mathrm{s}$. The residual acceleration vector in the $x$ - $z$-plane and transient bubble displacement with time are provide at right. A stationary (non-migrating) gas slug is seen in the transport tube above the migrating bubble. The spherical bubble inside the test chamber has a diameter of $6.25 \mathrm{~mm}$ and moves $33 \%$ slower and in the opposite direction than the bubble in the transport tube. The bubble displacement data for $30<t<$ 40s is missing due to a momentary camera obstruction.

Because gas absorption in the test fluid is a strong function of pressure, the diffusive time scale, from Table 5, for air absorption into the PDMS $\left(t \sim R^{2} / D=8\right.$ to $\left.32 \mathrm{~min}\right)$ was used to determine an equilibrium time of $30 \mathrm{~min}$. A wait time of $60 \mathrm{~min}$ was chosen for the system to equilibrate. Valves 1 and 2 are then sealed and the air in the test chamber and transport tube is compressed using the reservoir piston by turning the control knob $\mathrm{CW}$ until the over-pressurization clutch O-ring slips at approximately $4420 \mathrm{mBar}$, halting 
further pressurization. Once the compression is complete and a spherical bubble is established, the bubble displacement is tracked in time.
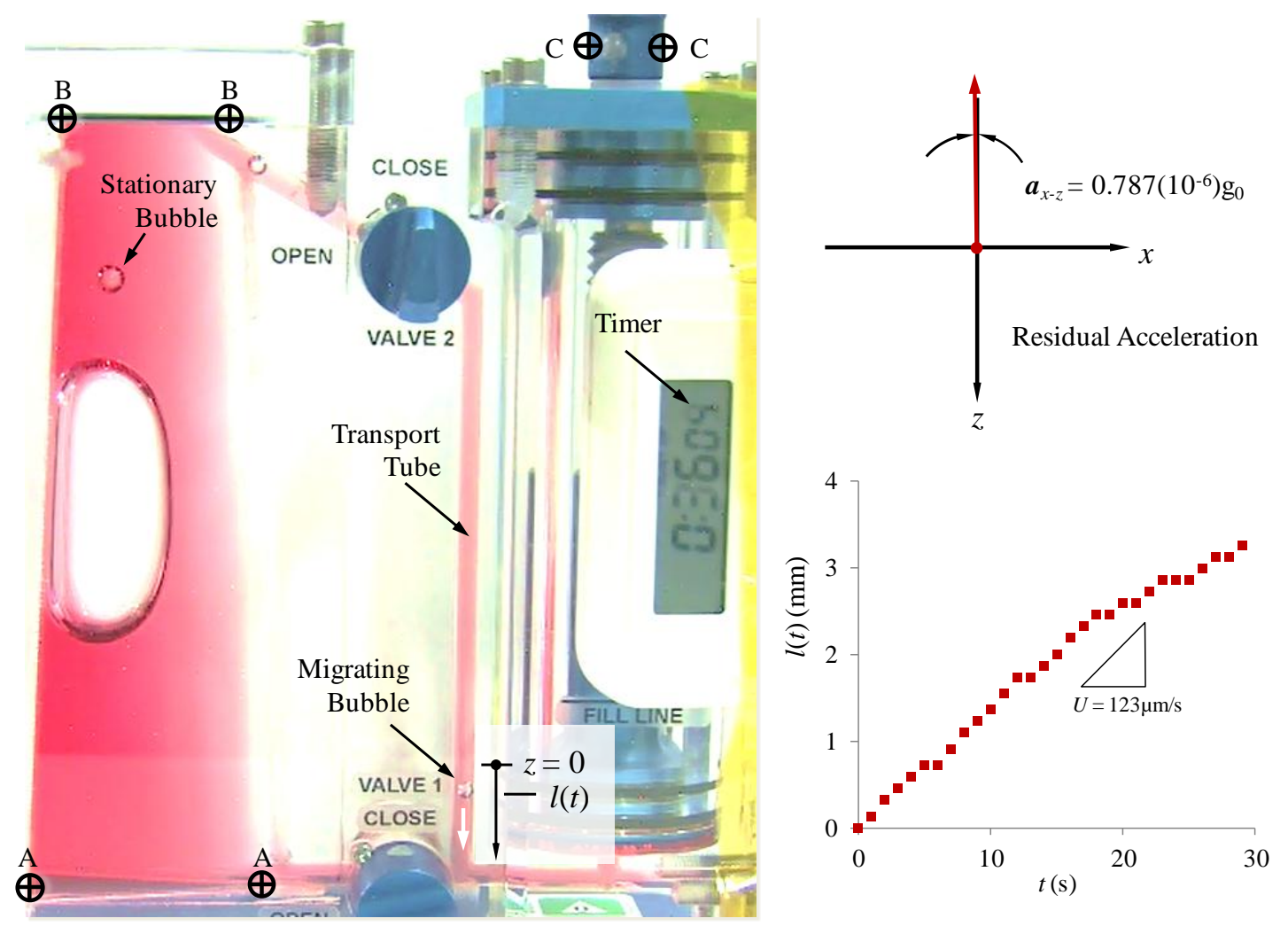

Figure 9. Pairs of points A, B, and C are selected with known distances to compute the scale factor field $\operatorname{SF}(x, y)$ for test ICF1-3. The timer is used to account for loss of video time stamp data during the HD video downlink. Behind the timer is the fluid reservoir with piston fully dispensed. A $3.32 \mathrm{~mm}$ diameter bubble is identified moving downward at $123 \mu \mathrm{m} / \mathrm{s}$ toward Valve 1. Residual acceleration vectors in the $x-z$-plane and transient bubble displacements are shown at right.

\subsection{Preliminary Comparisons of Analytic, Numerics, and Experiments}

\subsubsection{Local Acceleration Measurements}

The low-acceleration environment present on the ISS is the first suspect for a driving force for the bubble motion. Because of the disbelief in our original observations a significant effort was made to assess the orientation, magnitude, and spectrum of the ' $g$ environment' during the experiments. Only the results are summarized here with further 
details provided in Appendix $\mathrm{C}$ with references cited. The acceleration data is measured onboard by the 3-axis Microgravity Acceleration Measurement System (MAMS). MAMS measures low frequency accelerations $<1 \mathrm{~Hz}$ caused primarily by aerodynamic drag, vehicle rotations, gravity gradient effects and vents of air and water. The MAMS data is processed with an adaptive trimmed mean filter to provide an estimate of the quasi-steady acceleration environment, considered to be signals with frequency content below $0.01 \mathrm{~Hz}$.

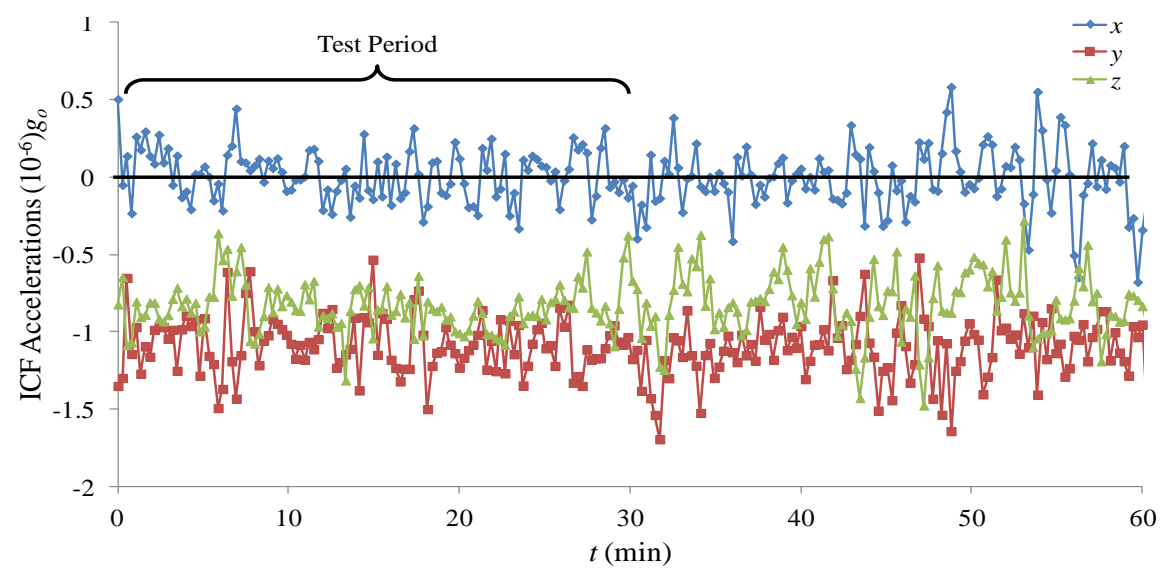

Figure 10. Corrected ISS MAMS data during the ICF1-4 experimental run beginning at GMT 2010252/08:00. Unlike the other tests, the $x$-axis is perpendicular to the plane of the vessel, but similar to the other tests with the positive $z$-axis points 'down' along the transport tube as sketched in Figs. 1618. The primary science of the ICF1-4 operations as well as the bubble migration occurred between 0 and 30min.

The quasi-steady acceleration magnitudes and orientations at the location of the experiments are computed based on displacements from the ISS center of mass and the orientation of the experiment with respect to the standard Local Vertical Local Horizontal $x-y-z$ reference frame (LVLH). Figure 10 displays sample acceleration levels for the ICF1-4 experiment run, where in this case the $x$-axis acts positive normal inward to the front face of the test cell, but the $z$-axis acts positive downward along the transport tube as sketched in Figure 7 through Figure 9. For this particular example, during the period of bubble migration $0 \leq t \leq 30 \mathrm{~min}$, the most pertinent $z$-accelerations are 
$-8.20\left(10^{-7}\right) \pm 1.68\left(10^{-7}\right) g_{o}$. The $x$ - and $y$-axis accelerations are $-2.22\left(10^{-9}\right) \pm 1.62\left(10^{-7}\right) g_{o}$ and $-1.09\left(10^{-6}\right) \pm 1.52\left(10^{-7}\right) g_{o}$, respectively, where $g_{o}$ is the magnitude of terrestrial gravity and the uncertainties are standard deviations.
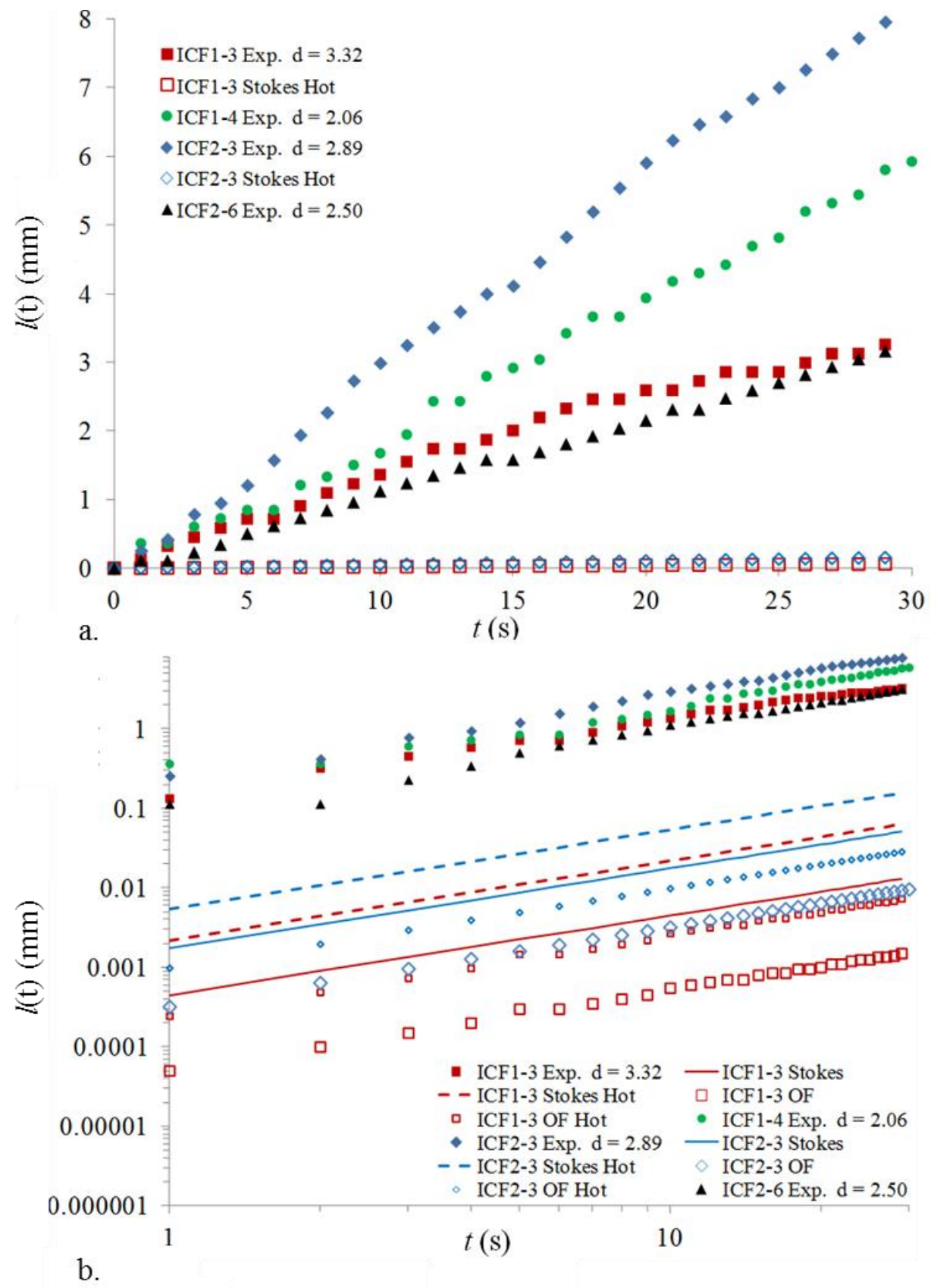

Figure 11. a. ISS experimental data for bubble migration is compared to isothermal Stokes flow and numerical flow solutions for worst case maximum temperature conditions. Each data set shows linear behavior $l(t)=a t$ with goodness of fit $R^{2} \geq 0.98 . \mathrm{b} . \ln l=\ln t+\ln a$ for the data shown in a. 


\subsubsection{Isothermal Stokes Flow Bubble Velocity Predictions}

A list of dimensionless groups for the bubble motion is compiled in Table 6. Reynolds numbers for the tests are found between 0.002 and 0.18 . Capillary numbers $<2\left(10^{-4}\right)$ suggest that distortion is negligible and the bubbles can be considered spherical. Assuming Stokes flow with $\operatorname{Re}<<1$, the balance between buoyancy and viscosity for a spherical bubble in an infinite medium [23] predicts a terminal velocity vector

$$
\boldsymbol{U}_{\text {st }}=\frac{2(1+\lambda)}{3(2+3 \lambda)} \frac{\left(\rho_{g}-\rho_{l}\right) \boldsymbol{a} R^{2}}{\mu_{l}}
$$

where $R$ is the bubble radius, $\mathbf{a}$ is the acceleration vector, $\mu_{l}$ is the dynamic viscosity of the host liquid, $\rho_{l}$ and $\rho_{g}$ are the respective densities of the liquid and gas, and $\lambda \equiv \mu_{g} / \mu_{l}$ is the viscosity ratio. Assuming an infinite medium, $|\mathbf{a}| \approx 10^{-6} g_{o}$, and $r=1 \mathrm{~mm}$, the settling velocity should be on the order of $0.1 \mu \mathrm{m} / \mathrm{s}$ and $1 \mu \mathrm{m} / \mathrm{s}$ for the 20 and $2 \mathrm{cSt}$ fluids at $25^{\circ} \mathrm{C}$, respectively, and values are listed in Table 6. Values are also computed and listed for viscosities evaluated at the maximum compression temperature. The values differ by as much as 6.5-fold. Confinement effects of the tube will have a further retarding influence on the drift velocity, but values computed in this manner serve as approximations of bubble velocity due exclusively to a local acceleration vector. The Stokes flow approximations are plotted in Figure 11 for cases ICF1-3 and ICF2-3. Under-predictions compared to the experimental results are significant: as observed in Figure 11 and Table 6, $U_{\text {exp }}$ is 276-fold greater for ICF1-3 and 143-fold greater for ICF2-3. It does not appear that gravity is the motive force although the flows due tend in the direction of the buoyant force. 
Table 6. Migration velocities for each experiment and relevant non-dimensional groups. Values for liquid and gas are provided where appropriate. Velocities are listed in $\mu \mathrm{m} / \mathrm{s}$. $\beta$ is the thermal expansion coefficient.

\begin{tabular}{|c|c|c|c|c|c|c|}
\hline Quantity & Definition & ICF1-3 & ICF1-4 & ICF2-3 & ICF2-6 & ICF1-8 \\
\hline$U_{\text {exp }}, 25^{\circ} \mathrm{C}$ & & 123 & 194 & 248 & 108 & 15.4 \\
\hline$U_{s t}, 25^{\circ} \mathrm{C}$ & & 0.445 & 0.237 & 1.74 & 1.02 & 0.485 \\
\hline$U_{s t}, 190^{\circ} \mathrm{C}$ & & 2.17 & 1.16 & 5.35 & 3.14 & 2.36 \\
\hline$U_{O F}, 25^{\circ} \mathrm{C}$ & & 0.051 & -- & 0.32 & -- & -- \\
\hline$U_{O F}, 190^{\circ} \mathrm{C}$ & & 0.25 & -- & 0.98 & -- & -- \\
\hline Bo & $(\Delta \rho) a R^{2} / \sigma$ & $9.7\left(10^{-7}\right)$ & $6.5\left(10^{-7}\right)$ & $5.7\left(10^{-7}\right)$ & $2.9\left(10^{-7}\right)$ & $2.1\left(10^{-6}\right)$ \\
\hline $\mathrm{Re}_{1}$ & $U R / v_{l}$ & 0.01 & 0.01 & 0.18 & 0.07 & .002 \\
\hline $\mathrm{Pr}_{1}$ & $v_{l} / \alpha_{l}$ & 260 & 260 & 23.9 & 23.9 & 260 \\
\hline $\operatorname{Pr}_{g}$ & $v_{g} / \alpha_{g}$ & 0.71 & 0.71 & 0.71 & 0.71 & 0.71 \\
\hline $\mathrm{Pe}_{1}$ & $U R / \alpha_{l}$ & 2.6 & 2.6 & 4.3 & 1.67 & 0.48 \\
\hline $\mathrm{Ma}_{1}$ & $-\sigma_{T}(\Delta T) R / \alpha_{l} v_{l}$ & $7.5\left(10^{6}\right)$ & $4.6\left(10^{6}\right)$ & $8.5\left(10^{7}\right)$ & $7.4\left(10^{7}\right)$ & $1.1\left(10^{7}\right)$ \\
\hline $\mathrm{Ca}_{1}$ & $\mu_{l} U / \sigma$ & $1.1\left(10^{-4}\right)$ & $1.8\left(10^{-4}\right)$ & $2.6\left(10^{-5}\right)$ & $1.0\left(10^{-5}\right)$ & $1.4\left(10^{-5}\right)$ \\
\hline $\mathrm{Ra}_{1}$ & $a(\Delta T) \beta_{l} R^{3} / \alpha_{l} v_{l}$ & $3.1\left(10^{-3}\right)$ & $1.3\left(10^{-3}\right)$ & $2.0\left(10^{-3}\right)$ & $8.8\left(10^{-4}\right)$ & $9.8\left(10^{-3}\right)$ \\
\hline $\mathrm{Ra}_{\mathrm{g}}$ & $a(\Delta T) \beta_{g} R^{3} / \alpha_{g} v_{g}$ & $4.7\left(10^{-5}\right)$ & $2.0\left(10^{-5}\right)$ & $2.4\left(10^{-5}\right)$ & $1.0\left(10^{-5}\right)$ & $1.5\left(10^{-4}\right)$ \\
\hline
\end{tabular}

\subsubsection{Isothermal CFD Bubble Velocity Predictions}

To quantify the confinement effects numerical computations using OpenFOAM [24] (OF) are conducted to model the bubble migration in the transport tube. The flows are solved using the bubbleFoam solver which uses the Euler-Euler [25] solution method for a twophase, incompressible, isothermal flow with dispersed gas phase.

Computations for 30s of physical time require 1-3 days of computational time on an 8 core $3.4 \mathrm{GHz}$ processor with $16 \mathrm{~GB}$ of ram. The OpenFOAM results are also presented in Figure 11 where it is observed that the confinement effect of the transport tube on the 
bubble results in velocity reductions of $11 \%$ of the infinite medium Stokes prediction for the $3.32 \mathrm{~mm}$ diameter bubble of ICF1-3 $(\mathrm{Re}=0.01)$ and $18 \%$ of infinite medium Stokes prediction for the $2.89 \mathrm{~mm}$ diameter bubble of ICF2-3 $(\mathrm{Re}=0.18)$. Such lower velocities are expected with the increased confinement of the bubble [26]. OpenFOAM results are also listed and plotted for the reduced liquid viscosities at the maximum compression temperatures which result in as much as a 6.5 -fold increase in Stokes velocity, but not near enough to account for the two order of magnitude higher measured velocity.

The compressed bubble migration velocity measurements are between 253 and 2411 times greater than exclusively buoyancy-induced velocities in the confined tubes depending on which temperature the dynamic viscosity is evaluated. However, it is also clearly established that all bubbles migrate in directions of what would be considered the buoyancy-induced flow direction, with one exception to be discussed in the next section.

\subsubsection{Solutocapillary Mechanism}

Other possible mechanisms for the compressed bubble motion might be due to surface tension or recoil forces associated with gas dissolution due to the rapid change in pressure. With an increase of temperature and pressure, a dissolved gas concentration gradient develops around the bubble. A resulting surface tension gradient could cause bubble motion as well as could a gas momentum imbalance due to preferential dissolution. However, during the purposeful ICF2-6 test a special effort was made to reach equilibrium before compression (i.e., a $60 \mathrm{~min}$ wait period). Following the subsequent compression, the bubble migrated for $4 \mathrm{~min}$ before coming to rest. Once the bubble motion halted, the bubble dissolved completely into the PDMS liquid over a 
$15 \mathrm{~min}$ period without further migration. Thus, neither mechanism seems likely to produce the observed migration. Nonetheless, surface tension measurements are performed on Earth using 2 and 20cSt PDMS pressurized in air to 3740mBar and held for 2 hours. The liquid is then depressurized to atmospheric pressure 1014mbar and timedependent surface tension measurements are immediately recorded by a Dunouy Ring KSV Sigma 702 tensiometer for approximately 40min. A plot of this data is provided in Figure 12 which at first glance indicates a negligible dependence of surface tension on dissolved gas concentration. The insets on the figure expand the $\sigma$-scale and do reveal a slight decrease in $\sigma$ with time. Surprisingly, the small differences and the time over which they are detected are adequate to cause a surface tension gradient flow, since the bubble moves in the direction of lower concentration (lower surface tension).

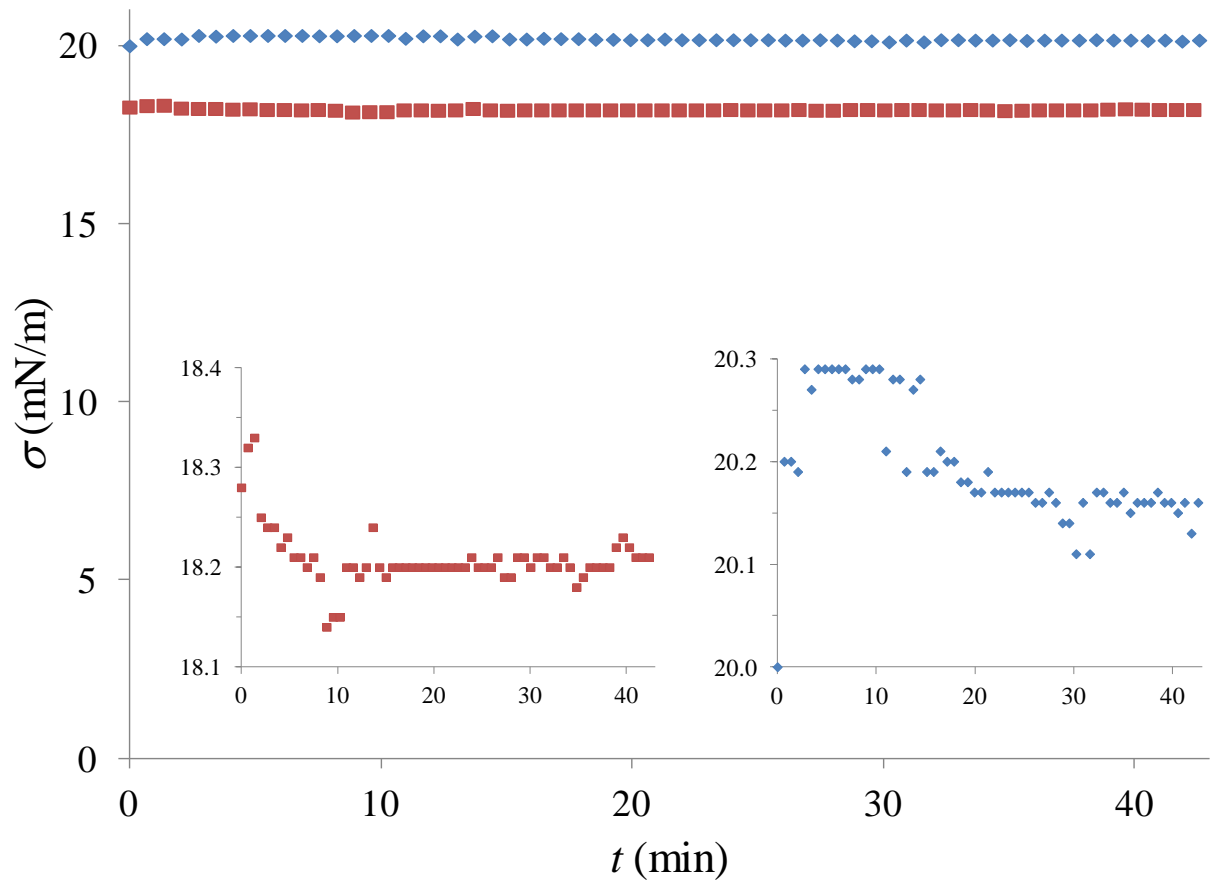

Figure 12. Transient surface tension measurement for $2 \mathrm{cSt}$ (red squares) and 20cSt (blue diamonds) PDMS as a function of dissolved gas concentration. The PDMS is initially pressurized with air to approximately $3740 \mathrm{mBar}$ for $2 \mathrm{hr}$ before initiating measurements at $1014 \mathrm{mBar}$. 
Another purposeful attempt to create compressed bubble migration, ICF1-8, took place which prompted further inquiry into pressurization as a mechanism for surface tension gradient migration. During the setup of the experiment and the positioning of the intended test bubble, an additional bubble was accidentally trapped in the transport tube and was seen to migrate after a 20s decompression which increased the bubble volume by a factor of 4. Prior to this experiment no bubble migration had been observed following a decompression.

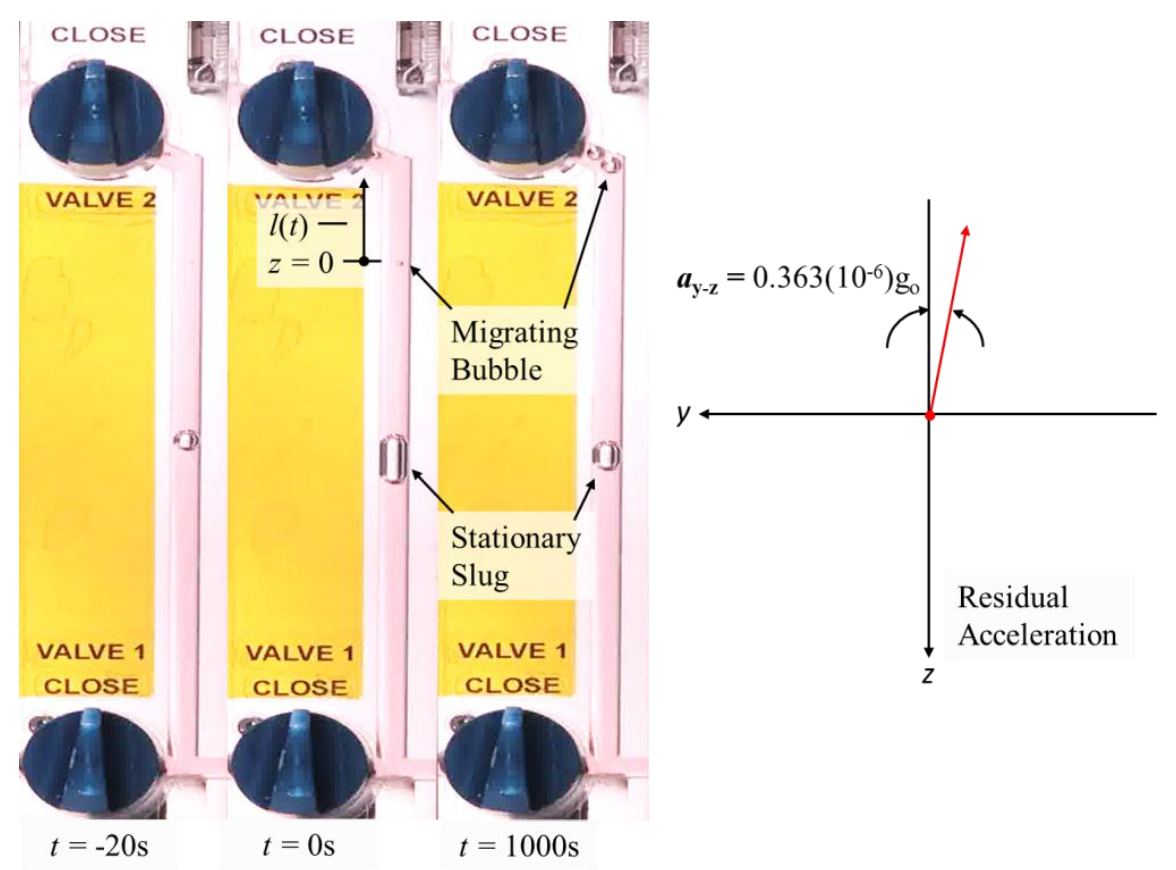

Figure 13. Close up of the ICF1-8 transport tube system immediately prior to decompression $(t=-20 \mathrm{~s})$, at the beginning of bubble migration $(t=0 \mathrm{~s})$, and just prior to the bubble coming to rest $(t=1000 \mathrm{~s})$. Note the increase in volume of the migrating bubble, while the stationary slug volume decreases over the same time period. Residual acceleration vectors in the $y$-z-plane are shown at right.

The bubble migrated for $16 \mathrm{~min}$ at a velocity of $15.4 \mu \mathrm{m} / \mathrm{s}$ until joining another bubble and coming to rest. Remarkably, the migrating bubble also increased in volume at a constant rate of $1.5\left(10^{-2}\right) \mathrm{mm}^{3} / \mathrm{s}$ (see Table 6) resulting in an increase of $13 \mathrm{~mm}^{3}$ (45-fold increase). Simultaneously, a stationary slug present in the transport tube was seen to 
decrease over the same time period by $42.87 \mathrm{~mm}^{3}$ ( $54.5 \%$ of original volume). Residual acceleration levels (see Figure 13) indicate the bubble migration velocity is still 32 times greater than the appropriate Stokes flow approximation. Additionally, it is important to note that unlike previously observed migrations, ICF1-8 bubble motion occurred in the opposite direction of buoyancy. This provides further evidence that density-driven convective currents are not responsible for observed bubble migrations.

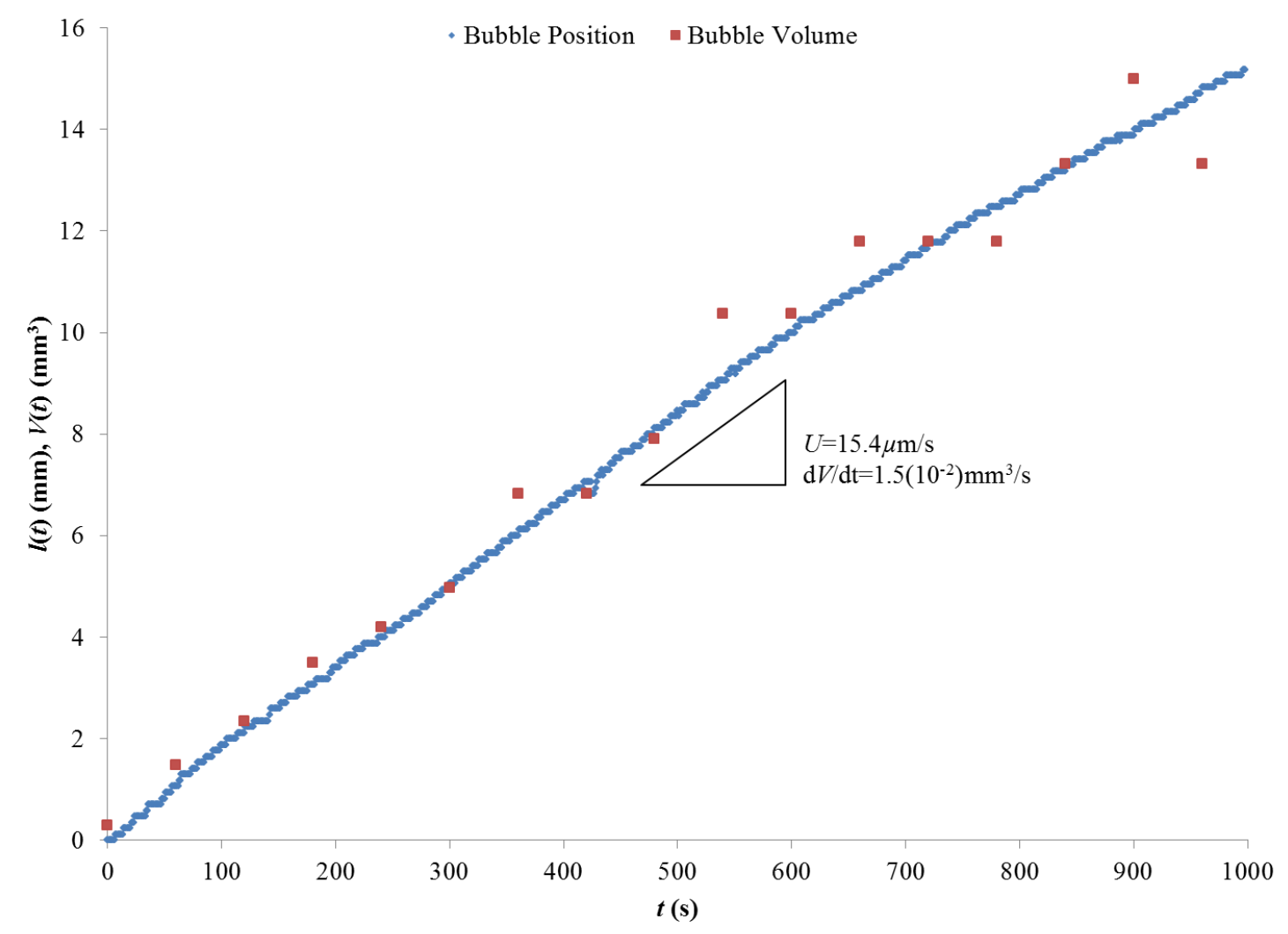

Figure 14. ICF1-8 decompressed bubble migration data. Migration velocity and rate of change of the bubble volume remain constant with $R^{2}$ goodness of fit values of 0.99 and 0.97 , respectively.

It is currently uncertain whether decompressed bubble migration operates by the same mechanism as compressed bubble migration, however following ICF1-8 observations it became prudent to investigate surface tension changes with respect to pressure change in a more controlled environment. 
The KSV Sigma 702 tensiometer was placed inside of a small vacuum chamber, seen in Figure 15, after adaptations were made to existing vacuum chamber equipment. Accommodations were made for the size of the tensiometer, and the need for vacuum seal compatible water, power, and data feeds (see Figure 16).

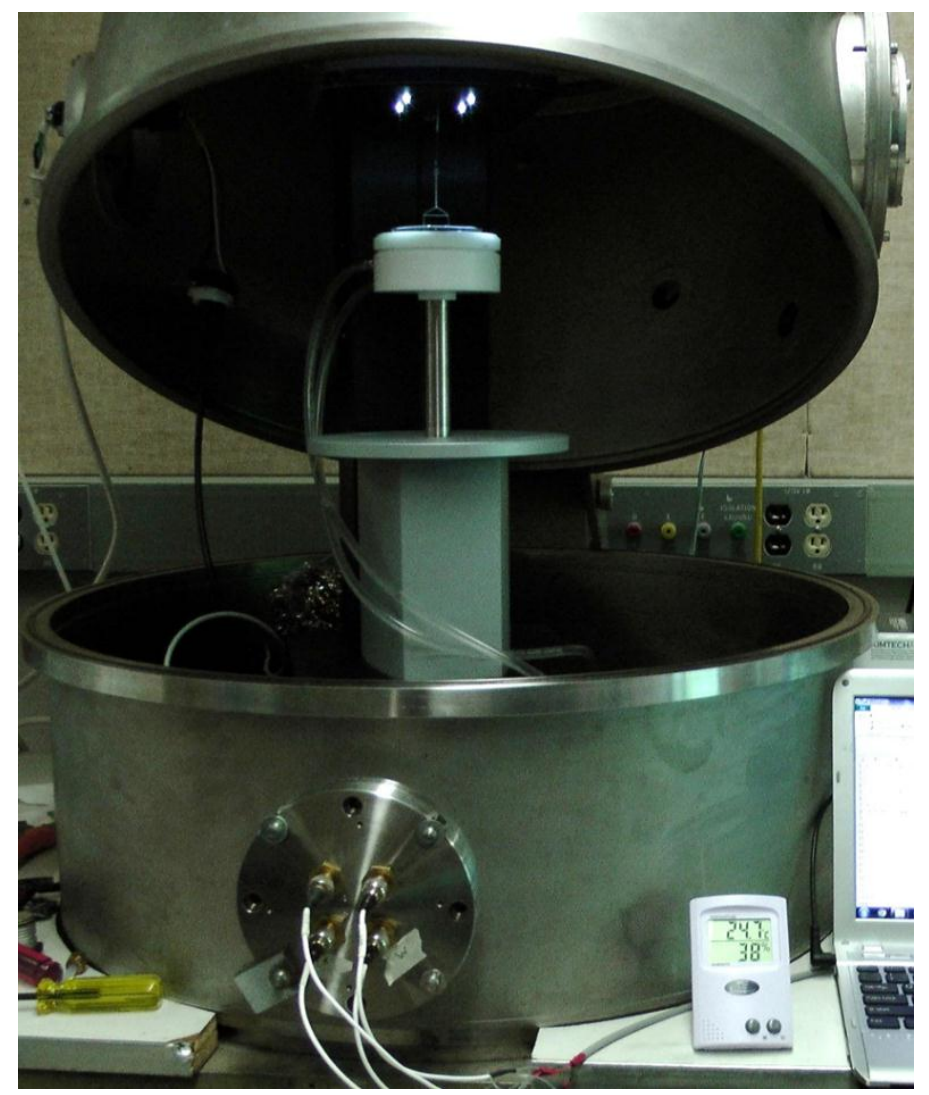

Figure 15. A vacuum chamber (open) with a KSV Sigma 702 tensiometer inside. Water lines can be seen attached to the white sample tray with a du Nuoy ring suspended above. 4 SHV cables are pictured coming out of a vacuum flange and are recombined into a USB cable for data acquisition.

Simple scaling shows that the surface tension gradient across the bubble needed to attain observed migration speeds needs to be on the order of $\Delta \sigma=10^{-3} \mathrm{mN} / \mathrm{m}$.

Unfortunately, the resolution of the KSV Sigma 702 tensiometer is of the same order so discerning a $\Delta \sigma$ of that magnitude may be difficult to detect above background noise. Despite this fact, it was hoped that larger $\Delta \sigma$ might be observable. 
A sample of 2cSt PDMS was placed in the tensiometer sample tray, which was kept at $17.5^{\circ} \mathrm{C}$ by a circulating water bath. Between each test the tensiometer was calibrated, and the du Nuoy ring was sterilized using a propane torch.
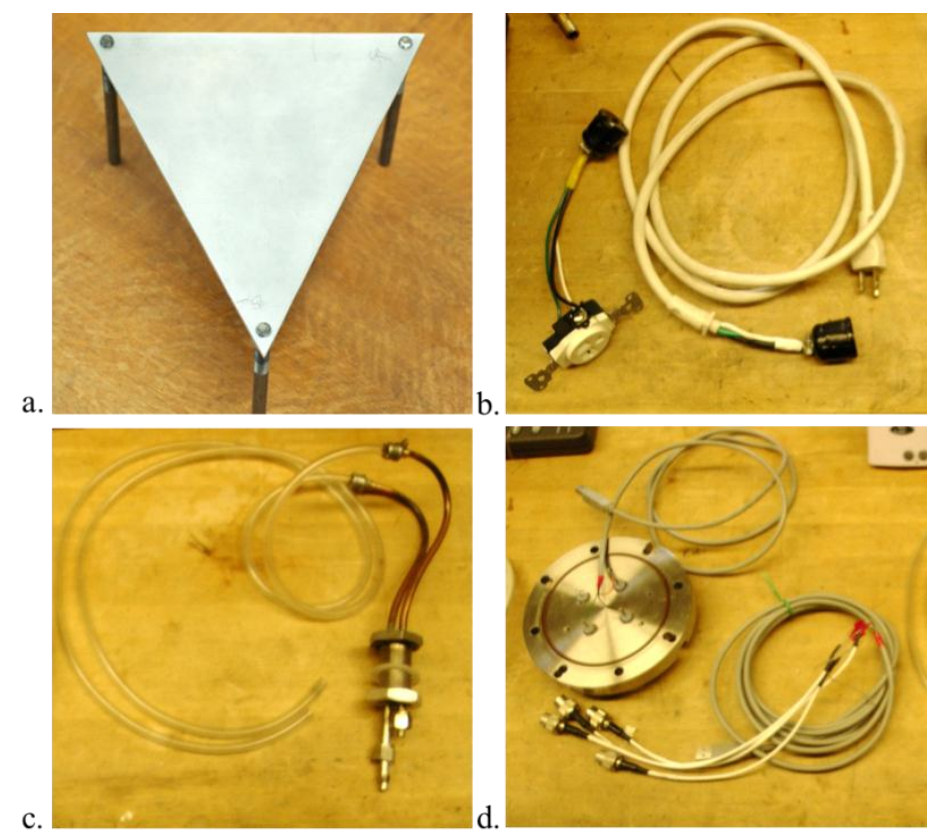

Figure 16. Materials needed to adapt the KSV Sigma 702 tensiometer for use in a vacuum chamber (VC). a. Aluminum plate and supports to elevate the tensiometer above preexisting VC equipment. $b$. Outlet and power cord soldered to octal pin plug connectors for power supply. c. VC argon gas feed with surgical tubing adapted for a water bath circulation feed. d. USB 2.0 cable spliced through 4 proprietary SHV connectors attached to a vacuum flange.

Two primary experiments were performed, seen in Figure 17 and Figure 18. First, the tensiometer was set to begin collecting readings as the vacuum chamber pressure was lowered to $22.86 \mathrm{kPa}$ and held for 30 minutes before returning to $101.32 \mathrm{kPa}$. A pressure of $22.58 \mathrm{kPa}$ was then held for 3 hours before returning to $101.32 \mathrm{kPa}$ at which point data collection began. Due to the programing of the tensiometer, test runs could collect a maximum of 63 test points over the period of approximately $45 \mathrm{~min}$. 


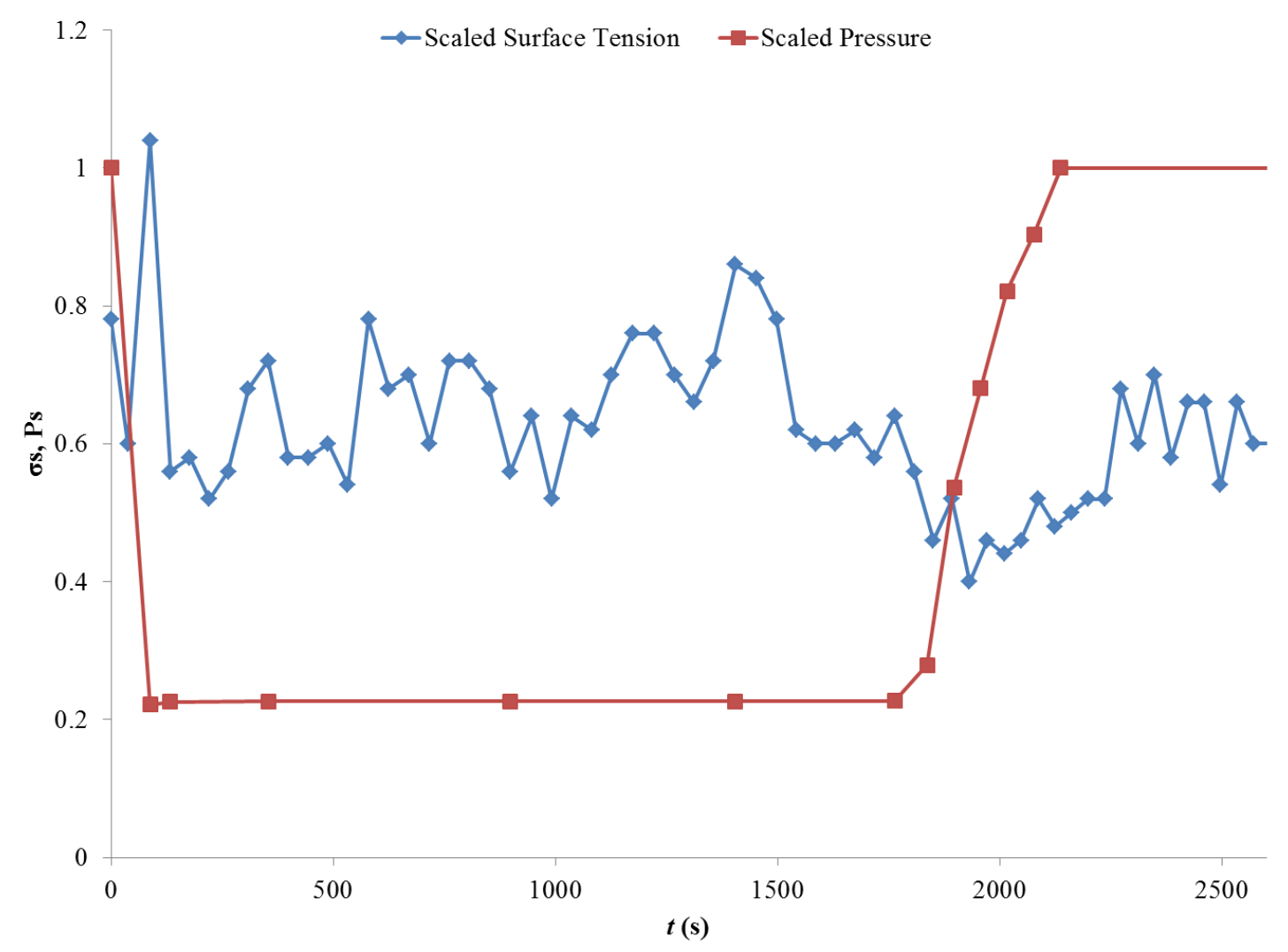

Figure 17. Scaled surface tension $\sigma_{\mathrm{s}}=\sigma_{\mathrm{exp}} / \sigma$ (solid diamonds) and pressure $\boldsymbol{P}_{\mathrm{s}}=\boldsymbol{P}_{\text {exp }} / \boldsymbol{P}_{\text {atm }}($ solid squares) graph of the first tensiometer measurements on 2cSt PDMS. No clear response in the surface tension due to pressure change is visible above background noise.

No detectable change in the surface tension can be seen in either the dynamic pressure change test (Figure 17), or the long duration depressurization/re-pressurization test (Figure 18).

At this point, there is no conclusive evidence showing a surface tension response to dynamic pressure changes. Unfortunately, unavoidable background noise due to environmental vibration or limitations in the resolution of the tensiometer could be obscuring more sensitive $\Delta \sigma$. Efforts may be made in the future to further reduce background noise and utilize a tensiometer with increased resolution. 


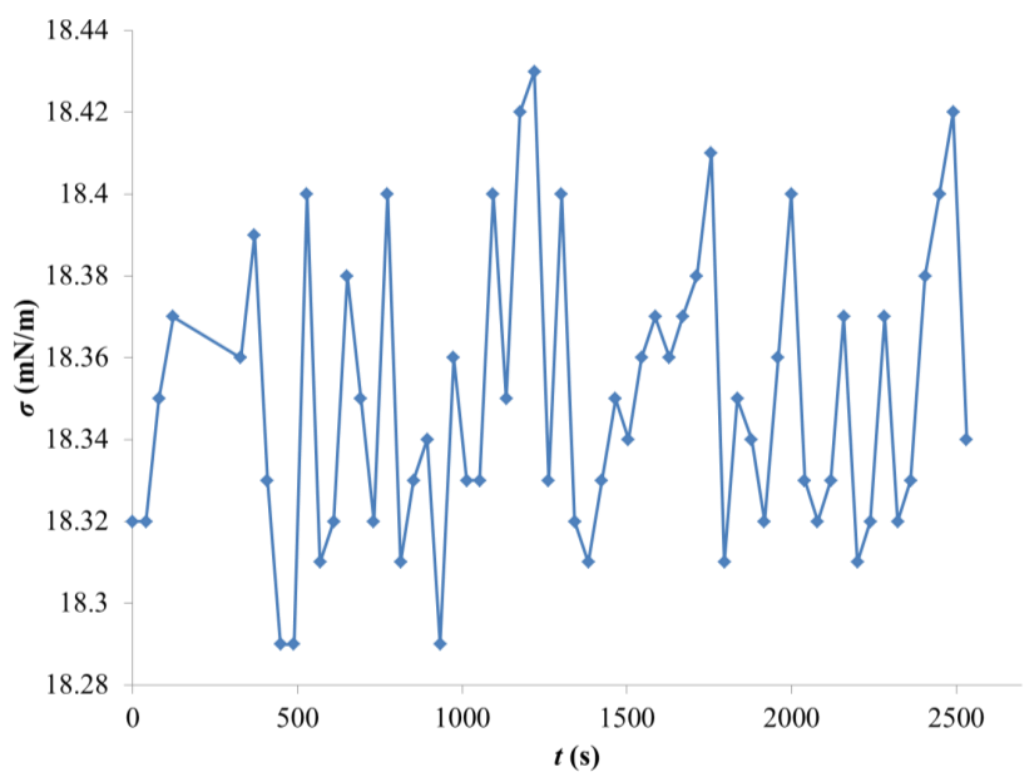

Figure 18. Surface measurements taken at $101.32 \mathrm{kPa}$ after the system was held at $22.53 \mathrm{kPa}$ for 3 hours. Again, no clear response in surface tension is seen above background noise.

So far a $\mathrm{d} \sigma / \mathrm{d} P$ on the order capable of generating the observed Bubble Migration velocities remains elusive, however so does the ability to rule it out with any certainty. Suggested avenues of research along these lines are provided at the end of this chapter.

\subsubsection{Thermocapillary Mechanism}

Assuming a simple adiabatic compression of a diatomic gas, the maximum temperature rise of the bubble is

$$
T_{f}=T_{i} \Delta V^{2 / 5},
$$

where $\Delta V \equiv V_{\mathrm{i}} / V_{\mathrm{f}}$ is the volumetric compression ratio and $T_{\mathrm{i}}$ and $T_{\mathrm{f}}$ are the absolute temperature of the initial and final states, respectively. Bubble compression ratios between 1.5 and 3 can be achieved with the ICF hardware. Thus, for air, temperature differences between the bubble and surrounding liquid can be as high as $52^{\circ}$ to $165^{\circ} \mathrm{C}$. Such a $\Delta T$, if to some degree manifest as a bubble surface temperature gradient, could in 
turn lead to surface tension gradients leading to well-established thermocapillary bubble motion $[27,28,29]$.

Local acceleration is effectively ruled out as the driving force for the flow. However, the migration does preferentially occur along vectors common to the residual acceleration. One possible mechanism for the flow is sketched in Figure 19a, where a heated gas bubble in a cold uniform temperature liquid is displaced by a gravitational acceleration. The bubble motion produces a hot spot at the leading edge of the bubble as recirculating re-heated gas within the bubble bears upon the lead stagnation point maintaining an elevated temperature there. The free surface cools as it circulates to the rear stagnation point maintaining a cooler temperature there. Thus, once the surface temperature difference is 'kicked' into existence the bubble migration by thermocapillary forces is maintained as long as there is sufficient temperature difference between the bubble and liquid to sustain the flow. Such a mechanism has been anticipated by Ryazantsev and Rednikov and co-workers [30,31,32].

To further support such a claim we invoke additional dimensionless groups listed in Table 6. Low Reynolds numbers and high Prandtl, Péclet, and Marangoni numbers provide additional support for thermocapillary motion as a primary mechanism of bubble migration [27]. If indeed the mechanism is thermocapillary, the literature suggests that a thermocapillary-induced drag coefficient [32] may be determined

$$
A=-\frac{\left[m\left(\frac{3}{4}-\frac{3}{25} \mathfrak{\aleph}^{-1}\right)+1+\frac{3}{2} \lambda\right]}{\left[m\left(\frac{1}{4}-\frac{2}{25} \mathfrak{x}^{-1}\right)+1+\lambda\right]}
$$

where $æ \equiv \alpha_{g} / \alpha_{l}$ is the thermal diffusivity ratio and a modified Marangoni number 


$$
m=-\sigma_{T} \frac{q R^{3}}{\boldsymbol{a}(2+\chi) k_{l} \mu_{l} \alpha_{l}},
$$

and where $q$ is the heat source intensity per unit volume and $\chi \equiv k_{\mathrm{g}} / k_{1}$ is the thermal

conductivity ratio. The quasi-steady velocity may then be predicted as

$$
\boldsymbol{U}=\frac{\left(\rho_{l}-\rho_{g}\right) R^{2}}{3 \mu_{l} A} \boldsymbol{a}
$$

The coefficient $A$ can assume any real value and can either enhance an existing flow or act against it as sketched in Figure 19. If the bubble migration of the present observations are thermocapillary in nature the thermocapillary drag coefficient must be $-3 / 2<A<0$ in order to enhance the velocity along the residual acceleration vector. The forms of Equations 3.3-3.5 require the assumptions of an infinite medium, a small Péctlet number, and no time dependence of the internal heat mechanisms. None of these conditions are met by the present experimental conditions.

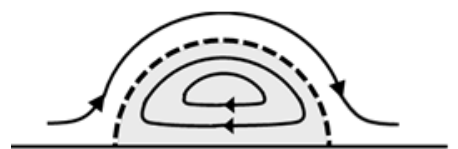

$A>-3 / 2$

a.

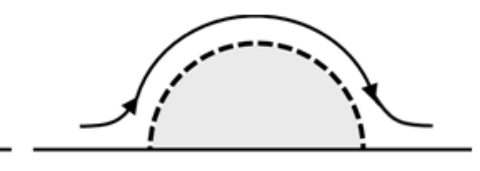

$A=-3 / 2$

b.

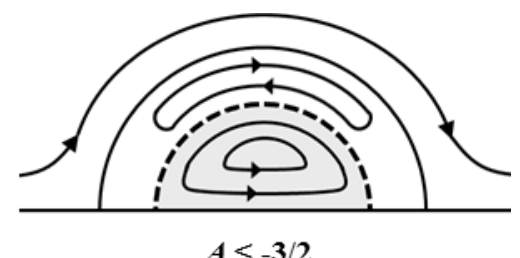

c.

Figure 19. Three outcomes of the thermocapillary drag coefficient on the flow pattern inside a heated thermocapillary bubble [32] from the bubble stationary reference frame: a. circulation inside the bubble, b. no flow inside the bubble whose motion becomes similar to Stokes flow around a solid sphere, and $c$. the circulation reversal inside the bubble with a boundary layer outside the bubble leading to additional drag.

It can be shown that a rough balance of thermocapillary driving force, buoyancy, and viscous resistance yields a velocity scale

$$
U \sim \frac{\sigma_{T} \Delta T}{\mu_{l}}, \frac{\Delta \rho a R^{2}}{\mu_{l}} \sim \frac{\sigma_{T} \Delta T}{\mu_{l}}\left(1, \frac{\Delta \rho a R^{2}}{\sigma_{T} \Delta T}\right) .
$$

The first group on the RHS appears as the temperature gradient capillary velocity scale. It 
is independent of bubble size. The second term is a ratio of the buoyancy force to

thermocapillary force. The latter increases significantly with bubble radius $R$, which leads one to expect that the thermocapillary-driven migration will be dominated by buoyancy for large bubble sizes.

One strong counter argument to the above thermocapillary concept is that the liquid surface temperature is controlled by the liquid's low thermal diffusivity and that it should actually heat up as it passes over the bubble. The warmer liquid would then achieve its highest temperatures at the rear stagnation point effectively turning off the thermocapillary mechanism. While this may be true in an infinite medium, the current experimental conditions are influenced by an acrylic tube confining the liquid-bubble system.

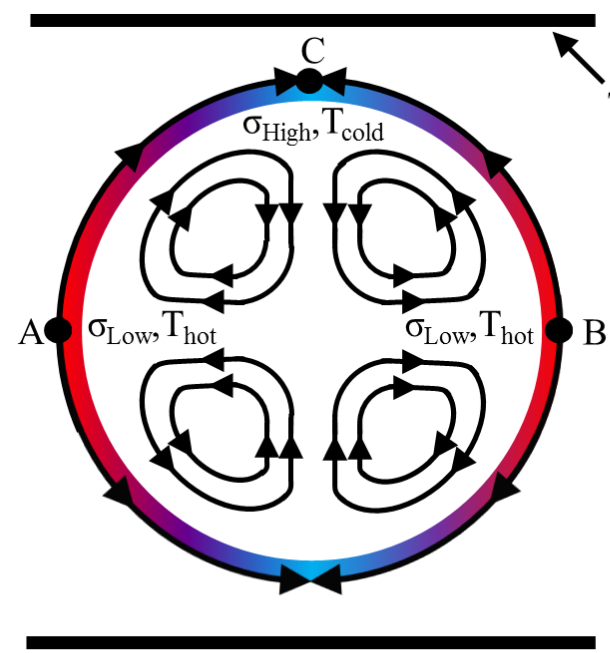

a.

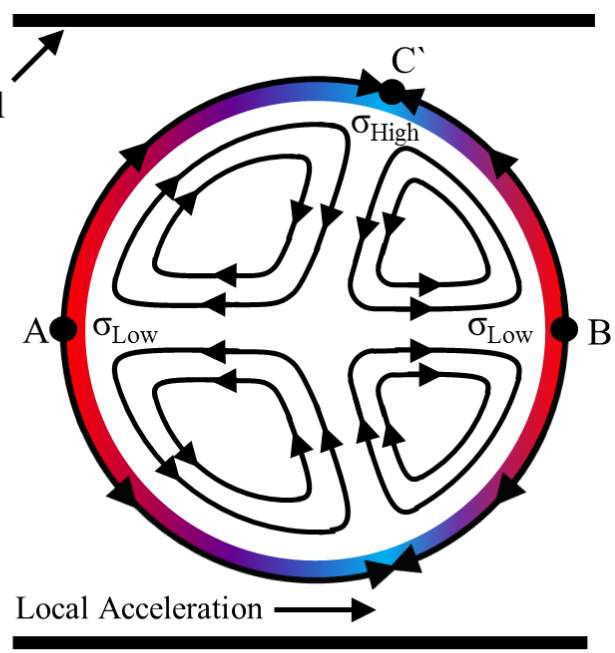

b.

Figure 20. Two configurations showing tube wall influence on surface temperature of a compressionheated bubble. a. points $A$ and $B$ have equal temperature due to symmetry while $C$ has the lowest temperatures due to wall influence. No motion occurs due to symmetry. b. symmetry is broken by a local acceleration. Point $\mathrm{C}$ moves to $\mathrm{C}^{\prime}$ and the integrated stress from $\mathrm{AC} \mathrm{C}^{\prime}$ is now greater than that between $\mathrm{BC}$ ' causing enhanced motion in the direction of buoyancy. Streamlines in the liquid are not shown.

It is possible that the thermal boundary conditions of the transport tube wall provide a 
favorable temperature gradient over the bubble interface. A theory of tube wall interaction is sketched in Figure 20a where a bubble with $T=T_{1}$ is at rest in a fluid at $T=$ $T_{0}$ where $T_{1}>T_{0}$. The bubble surface has its lowest temperatures near the tube wall and remains motionless due to symmetry. When symmetry is broken by local acceleration, as in Figure 20b, the 'cold spot' on the bubble will move towards the rear stagnation point. The integrated thermocapillary stress over the liquid-bubble interface perpetuates motion in the direction of buoyancy, but at a greatly increased velocity.

Another strong counter-argument to the compression heat-driven thermocapillary bubble motion idea is the time scale of temperature dissipation. The Rayleigh numbers $\mathrm{Ra}<<10^{3}$ listed in Table 6 confirm conduction as the primary mode of heat transfer. Solving the transient conduction equation [33] for a spherical air bubble of diameter $2.89 \mathrm{~mm}$ in an infinite liquid medium, from Error! Not a valid bookmark selfreference., thermal diffusivities $\alpha_{g}=k_{g} / \rho_{g} c_{p, g}=2.1\left(10^{-5}\right) \mathrm{m}^{2} / \mathrm{s}$ and $\alpha_{l}=k_{l} / \rho_{l} c_{p, l}=8.37\left(10^{-}\right.$ $\left.{ }^{8}\right) \mathrm{m}^{2} / \mathrm{s}$, it is clear that the heat transfer is liquid-side limited. The characteristic time for liquid conduction is estimated to be $t \sim R^{2} / \alpha_{l} \approx 18 \mathrm{~s}$ based on a $98 \%$ convergence requirement. Because temperature gradients on the order of $0.01^{\circ} \mathrm{C}$ over the diameter of the bubble are capable of producing the migration velocities observed, a 99.99\% convergence criteria might be employed yielding $t \sim 489 \mathrm{~s}$. These times present a problem in that the 'compressed bubble migration flows' are observed for up to 710s. From Error! Not a valid bookmark self-reference. the thermal diffusivity of the acrylic surrounding the transport tube is $\alpha_{\text {wall }}=9.74\left(10^{-8}\right) \mathrm{m}^{2} / \mathrm{s}$, a similar value to the liquid $\left(\alpha_{l}=\right.$ $\left.8.4\left(10^{-8}\right) \mathrm{m}^{2} / \mathrm{s}\right)$. It is possible that the boundary conditions of the transport tube alter the 
thermal time scale, but how significantly is yet to be learned.

\subsection{Directions}

Although additional work is required to establish the compressed bubble migration phenomena with greater certainty, the potential for exploiting pressure changes for the manipulation of two phase systems aboard spacecraft, or in low-g materials

manufacturing processes, remains a compelling prospect. Additional bubble compression experiments are planned for the CFE-2 test units currently onboard ISS. There is currently no plan to return the flight units to Earth and an additional 5 units will be shipped to the ISS by spring of 2013, any of which could yield to further bubble compression tests — inadvertently or on purpose.

Ground-based experiments should be performed to try and conclusively measure $\mathrm{d} \sigma / \mathrm{d} P$ to an accuracy capable of producing observed bubble migration velocities $\left(10^{-3} \mathrm{mN} / \mathrm{m}\right.$ over the diameter of the bubble) and the dependence of bubble velocity on the compression/decompression of micro-scale bubbles.

An optically transparent sealed container could be constructed which contains several connected tubes of known diameters and is partially filled with PDMS silicone oil. Using a pressure transducer, highly accurate measurements of pressure change in the system over time will be recorded. By then compressing/decompressing the system, and recording the silicone oil meniscus with an HD camera, image analysis software can be employed to measure slight changes in the height of the meniscus and thus the surface tension.

Additionally, an experiment could be designed to determine the change in velocity of 
micro-scales bubbles undergoing buoyancy when the system is pressurize/depressurized. Many studies have been conducted on the rise of bubbles due to buoyancy and temperature gradients $[28,29]$, however a search of the literature yields no research conducted on the change in the velocity of a rising bubbles under dynamic pressure changes. Using a thermally and optically transparent sealed apparatus filled with a liquid medium, hydrogen bubbles on the order of $1 \mu \mathrm{L}$ could be injected into the medium and its motion observed. The rise rate of the bubble and the increase in volume associated with the change in hydrostatic pressure is well known, but additional benchmarking should be performed. Once the buoyant velocity of the bubble is determined, the system should be pressurized/depressurized at the midpoint of the bubbles rise and any change in velocity noted. A IR camera chould be used to determine the temperature change of the bubble due to compression/decompression, and care should be taken so that the volume change of the bubble does not cause it to exceed capillary dominated regime.

Finally, numerical simulations should be conducted to determine if migration events seen during ICF experiments can be repeated using thermal or gas concentration gradients. The interFoam solver integrated into OpenFOAM [24] currently does not have the capabilities to simulate thermocapillay effects, however due to the programmable nature of the CFD program, efforts could be made to add surface tension gradient forces into the algorithm. Unfortunately, the mesh resolution required to capture the sensitivity of the surface tension gradient will require a large amount of processing power and computational runtimes, though employing computing clusters would help alleviate the issue. 


\section{Chapter 4}

\section{Blade-Bound Droplet Stability}

\subsection{Background}

As humanity spends more time in space and private companies are venturing into space craft manufacturing, it is becoming more important to have control over multi-phase systems such as PMD’s (propellant management devices), and waste management systems [13]. In low gravity environments liquid drops or bubbles preferentially become bound to surfaces as opposed to floating free in the medium. Recently this became a problem when an Extra Vehicular Activity (EVA) to install new experiments and fixtures to the exterior of the International Space Station (ISS) was cut short due to a faulty sensor [9]. It was speculated that a fluid droplet had become bound to the surface of the sensor which caused its malfunction. The surface energy of such a wall-bound drop is at a local minimum, requiring some form of perturbation to dislodge it from the surface. Knowledge of the perturbations required to dislodge such drops provides a valuable design guide.

One such perturbation is provided by gravity. The Bond number $\left(\mathrm{Bo}=\rho g L^{2} / \sigma\right)$ is a dimensionless number which provides a measure of gravity's effect in an otherwise capillary dominated system. For the purpose of this experiment $L=V^{1 / 3}$, where $V$ is the volume of the bound drop.

Utilizing SE-FIT [10], a graphical user interface developed at Portland State University for K.A. Brakke's Surface Evolver [11], numerical simulations are being 
conducted for a range of gravity vector directions to determine the critical Bond number $\mathrm{Bo}_{\mathrm{cr}}$ at which so called 'Wall-Edge-Vertex-Bound drops' (a.k.a. drops on blade trips or drops on leaf tips which they resemble) will detach from the surface. Expanding on research conducted by Chen et al. [9], which focused on drops bound by the edge of 2-D planes, a wedge with a corner half-angle $\alpha$ of $60^{\circ}$ is investigated and thousands of simulations are performed for wedges of varying $\alpha$ and $\theta$ with $g$ in the plane of the blade and pointing off the tip. A schematic of the problem geometry is presented in Figure 21.

In this chapter, the current numerical results are benchmarked against previous numerical results and quantitative comparisons are made against laboratory experiments. Additionally, a previously uninvestigated phenomenon of residual droplet volume after the critical breakoff point is presented as is an intriguing 'dry leaf tip' phenomenon observed for a range of the critical geometric and wetting parameters.

With the support of other researchers, and as part of a graduate class in capillary phenomenon, thousands of simulations with an array of corner half-angles, contact angles, and g-orientations are conducted and will be submitted shortly for publication [34].

\subsection{Description of Numerical Approach and Benchmarking}

Since the early 1990's Brakke's Surface Evolver [11] (SE) algorithm has become a powerful asset in the computational investigation of equilibrium capillary surfaces. The algorithm seeks to minimize the total energy of the system, including the surface, gravitational, and rotational energies. During computation, perturbations to the system are added to the evolving surface which is then tested by the algorithm to see if it returns 
to an equilibrium state. SE examines the Hessian matrix's eigenvalues to determine the state of the system. The system is deemed to be at a stable equilibrium if each eigenvalue is positive, and conversely, is deemed unstable if any negative values exist. SE-FIT uses SE as a kernal within its larger graphical user interface (GUI) environment. Using the Parametric Sweep Function (PSF) batch process, an automated script which is integrated into $S E-F I T$, the gravity vector out of the plane $\varphi$ is iterated from $0^{\circ}$ (pendent drop) to $175^{\circ}$, in $5^{\circ}$ increments (See Figure 22) and the critical Bond number is then determined.
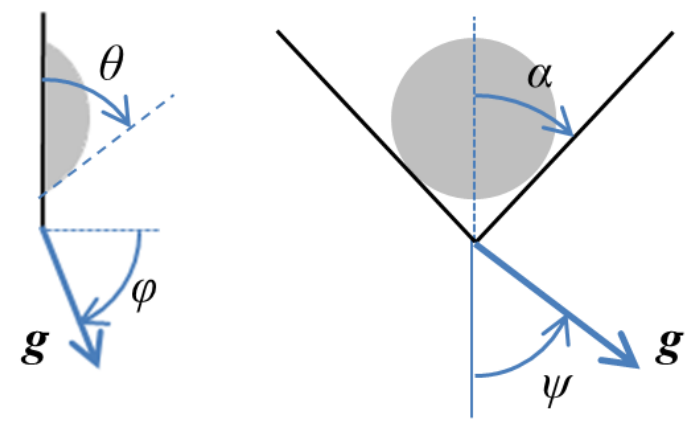

Figure 21. A side-on profile of a blade (left) with the contact angle $\theta$ of a droplet adhered to the surface, and a top-down image (right) of the corner half-angle $\alpha$ of a blade. The $g$-vector is shown with components in the plane $\psi$ and out of plane $\varphi$ of the wedge.

Using the stability routine described above for each increment the PSF batch process determines the $\mathrm{Bo}_{\mathrm{cr}}$ at which point the drop becomes unstable and detaches from the surface. A coarse mesh, with a maximal dimensionless edge length of 0.5 , was used to determine a rough estimate of the $\mathrm{Bo}_{\mathrm{cr}}$, while a finer mesh with a maximal edge length of 0.1 was used to further refine the values. Both surfaces were deeply converged using a total energy variation of less than $10^{-4}$. Runtimes on a $2.27 \mathrm{GHz}$ Intel i5 processor with 8 GB of RAM and a Windows 7 64-bit operating system ranged from 0.5 hours with the rough mesh, to 96 hours with the fine mesh. To benchmark the computation, a rough mesh was run for three fluids of constant contact angle $\theta=0^{\circ}, 60^{\circ}$, and $175^{\circ}$, see Figure 
22. In Table 7 the pendent drop cases are compared to previous simulations by Chen et al. [9]. It is seen in Figure 22 that the critical Bond number behaves expectedly. A local minimum is observed at $\varphi \approx 90^{\circ}$ (gravity is normal to the point of the wedge) and begins to rise towards infinity as the sessile drop case $\left(\varphi=180^{\circ}\right)$ is approached.

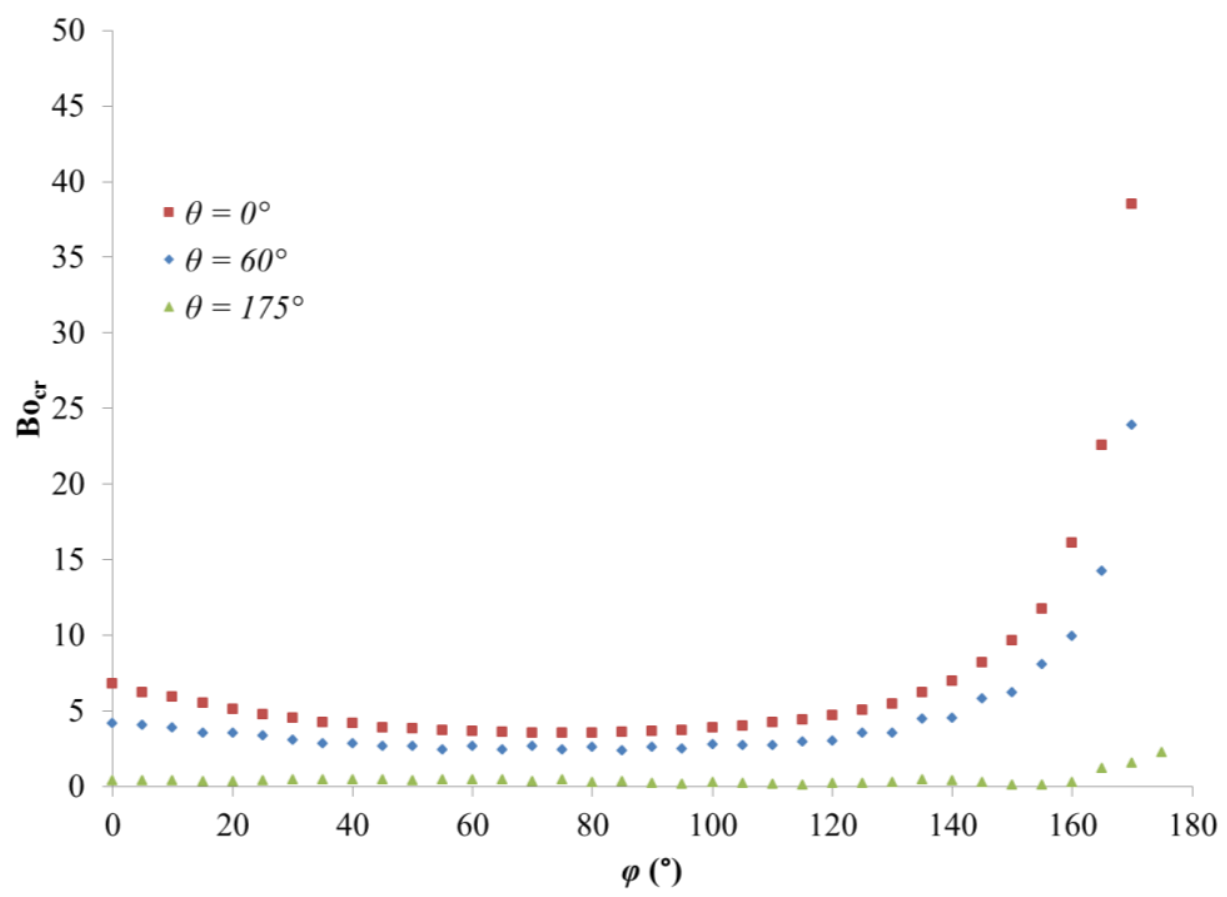

Figure 22. Graph of the rough mesh critical Bond number versus the angle of the gravity vector $\varphi$ for a blade with $\alpha=60^{\circ}$. Note the critical Bond numbers for the pendant drop case $\left(\varphi=0^{\circ}\right)$ as this is compared to previous numerical results in Table 7.

The results obtained from SE for the $0^{\circ}$ and $60^{\circ}$ contact angle pendent drop cases show excellent agreement with previous numerical results. However, the $175^{\circ}$ case shows a large discrepancy due to the convergence criteria of the rough mesh, indicating that the finer mesh should be used when greater resolution is required, such as in sessile drop cases. 
Table 7. Comparison of pendant drop simulations with previous numerical results. $\alpha=60^{\circ}$.

\begin{tabular}{|c|c|c|c|}
\cline { 2 - 3 } \multicolumn{1}{c|}{} & \multicolumn{2}{c|}{$\mathrm{Bo}_{\text {cr }}$} & \multicolumn{1}{c}{} \\
\hline$\theta$ & Wedge & $\begin{array}{c}\text { Chen et } \\
\text { al. [10] }\end{array}$ & $\begin{array}{c}\% \\
\text { Difference }\end{array}$ \\
\hline 0 & 6.80 & 7.1 & 4.32 \\
\hline 60 & 4.20 & 4.0 & 5.00 \\
\hline 175 & 0.42 & 0.1 & 320 \\
\hline
\end{tabular}

\subsection{Laboratory Experiments}

For simplicity of manufacturing, all blades used in this experiment were created from standard transparency sheets. The primary blade, with $\alpha=60^{\circ}$, is approximately 21 by $14 \mathrm{~mm}$ with the blade along one edge (see Figure 23). Other equipment used included a $10 \mu \mathrm{L}$ syringe and a mount capable of rotating through a full $360^{\circ}$. The drops were backlit with a diffuse LED light source and images were captured using a 38mm digital camera. NASA's Spotlight image analysis software [35], was used to confirm the fluid contact angle (Figure 23).

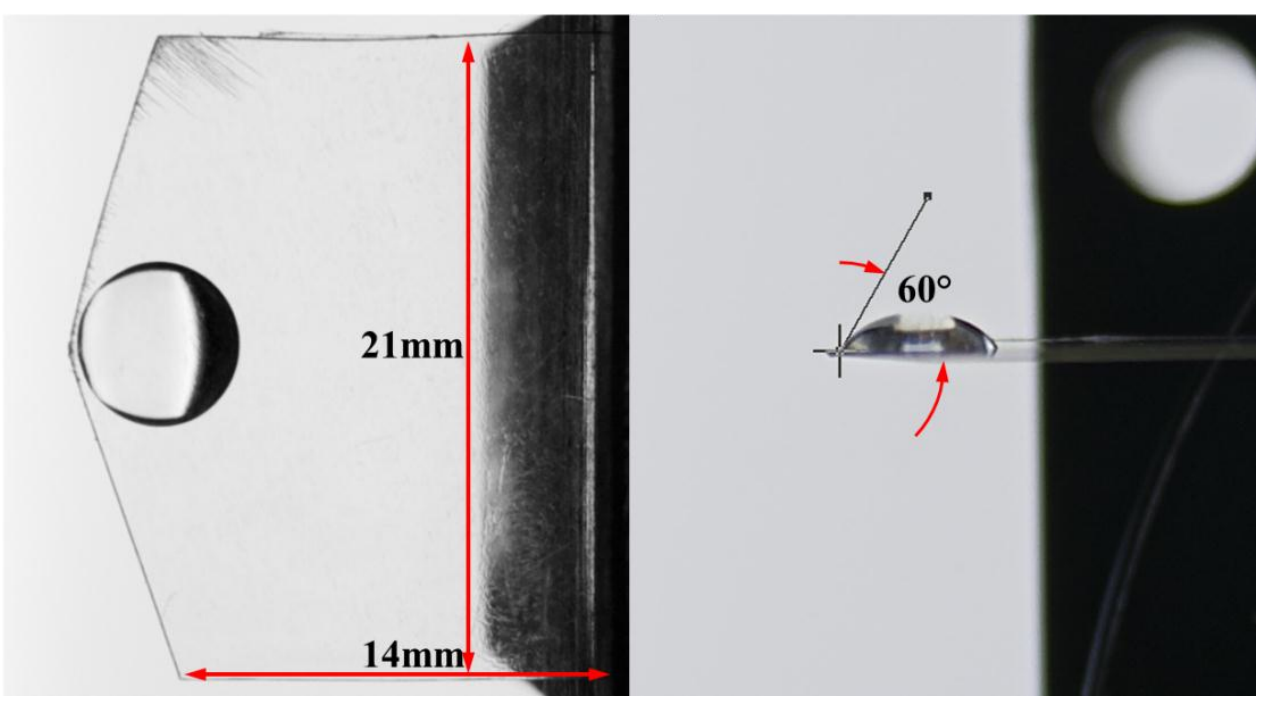

Figure 23. Image of the experimental setup and fluid contact angle for a $14.5 \mu \mathrm{L}$ drop. A contact angle of $60^{\circ}$ is confirmed using image analysis software (right). 
The transparency blades were washed in warm, soapy water, blown dry, rinsed in methanol ( $3 \mathrm{x})$, then coated $(2 \mathrm{x})$ with surface modifier FC-724, manufactured by the $3 \mathrm{M}$ company. The FC-724 coating ensures the $60^{\circ}$ contact angle condition.

\subsubsection{Wall-Edge-Vertex-Bound Drops $\left(\alpha=60^{\circ}\right)$}

With the wedge mounted, drops of a known volume, and thusly a known Bo, were deployed. The apparatus is gradually tilted until the drop falls from the surface representing an approximate range for the angle of detachment. The mount is then tilted back $5-10^{\circ}$, a drop of equal volume re-deployed, and the mount again tilted until the point of detachment. This method was employed to decrease the impact of contact angle hysteresis as the drop relocates from its original placement.

Table 8. Properties of the fluid used in the lab experiments. Contact angle is in degrees.

\begin{tabular}{|c|c|c|c|c|}
\hline Liquid & $\mu(\mathrm{kg} / \mathrm{m} . \mathrm{s})$ & $\rho\left(\mathrm{kg} / \mathrm{m}^{3}\right)$ & $\sigma(\mathrm{mN} / \mathrm{m})$ & $\theta \mathrm{w} / \mathrm{FC}-724$ \\
\hline $\begin{array}{c}10 \mathrm{cSt} \\
\text { PDMS }\end{array}$ & $9.35\left(10^{-3}\right)$ & 935 & 20.1 & 60 \\
\hline
\end{tabular}
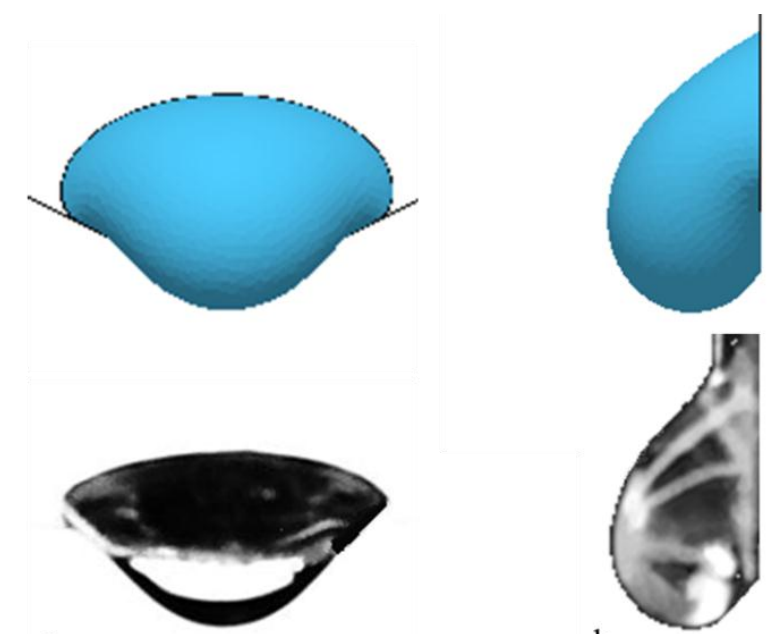

a.

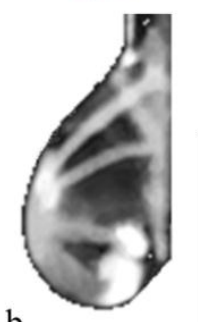

Figure 24. Comparison of SE drop profiles (above) versus experimental results. The profiles represented are a) $\mathrm{Bo}=5.9$ (left) and $\mathrm{Bo}=2.3$ (right). 
Drop volumes of $10,12,14,14.5,15,16,25$, and $50 \mu \mathrm{L}$ were investigated and repeated measurements were taken for droplets of $14.5 \mu \mathrm{L}$ and larger since any volume below that did not have a Bond number sufficient enough to detach from the surface. The fluid properties in Table 8 were used to calculate the Bo of the drops. It is important to note that the characteristic length being used $\left(L=V^{1 / 3}\right)$ is only an approximation and does not take into account any geometry or contact angle dependence. Future work will be done to calculate this value with greater accuracy.

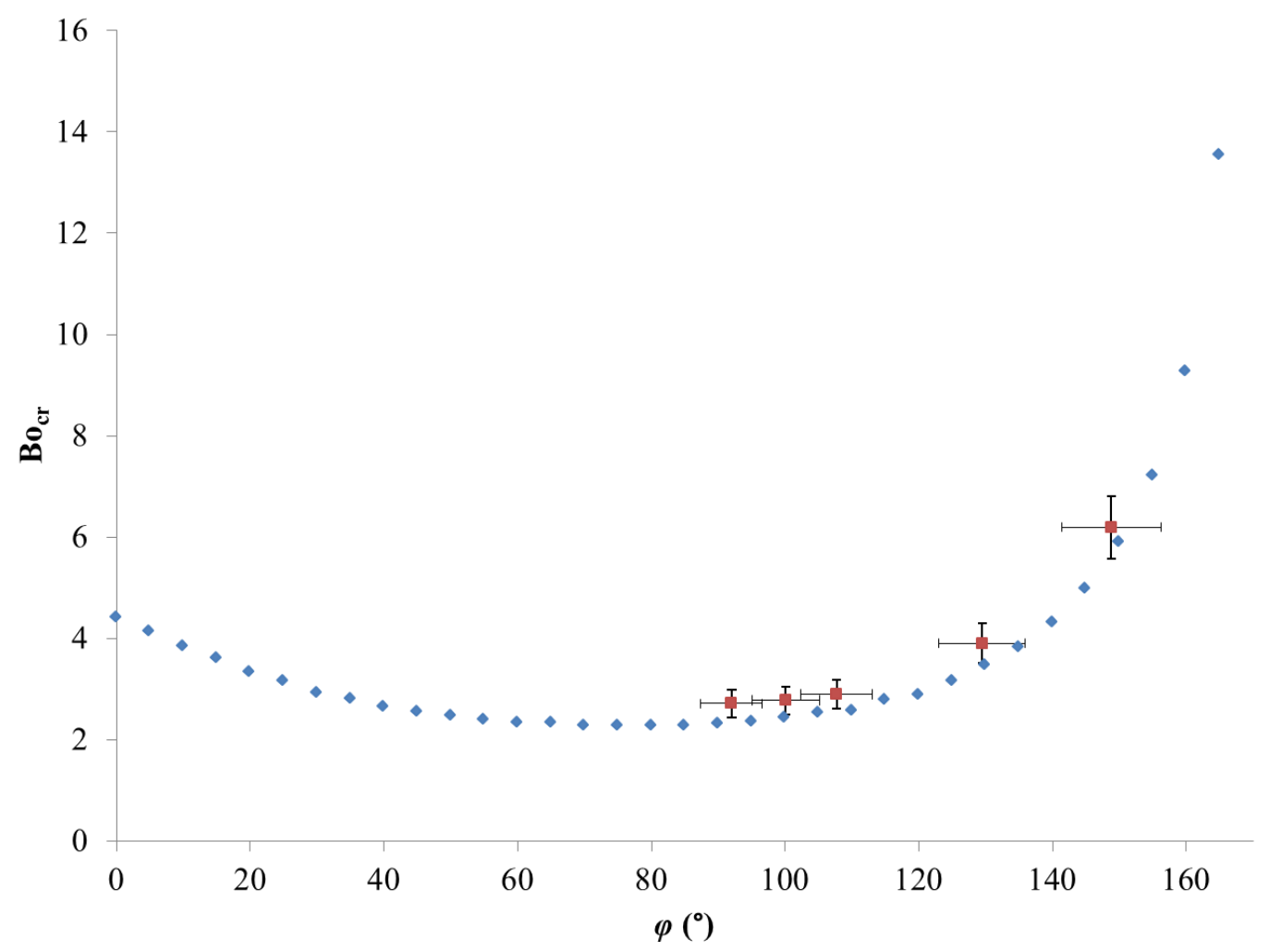

Figure 25. Finer mesh SE-FIT simulation versus experimental results for $\alpha=60^{\circ}$. Error bars represent $10 \%$ vertical error and $5 \%$ horizontal error.

Figure 24 shows the finer mesh SE-FIT simulation profiles of stable surfaces just prior to $\mathrm{Bo}_{\mathrm{cr}}$ compared to experimental surfaces of similar Bo. The drop profiles from $S E$ FIT agree favorably with experimental results making it clear that the numerical values of 
the $\mathrm{Bo}_{\mathrm{cr}}$ can be accurately compared to the experimental results. In Figure 25, the fine mesh SE-FIT simulation results are plotted alongside the experimental data. Once again, SE-FIT shows good agreement with the experimental results suggesting that numerical simulations of a Wall-Edge-Vertex-Bound drop could be employed as a powerful quantitative tool for system design.

\subsubsection{Residual Drops}

During the experiments it was noted that after a drop detached from the blade, some liquid would remain. After repeated experiments it was noticed that the volume of the 'Residual Drop' varied with the volume of the initial drop placed on the transparency blade such that the detached drop had a consistent volume (see Figure 27). This prompted further investigation into the phenomenon. The SE-FIT algorithm was set for the Bo = 2.7 equivalent to the $14.5 \mu \mathrm{L}$ drop volume and a value of $\varphi$ known to detach the drop. Figure 26 suggests how SE 'predicts' the establishment of a residual droplet despite the fact that SE is not a dynamic solver.

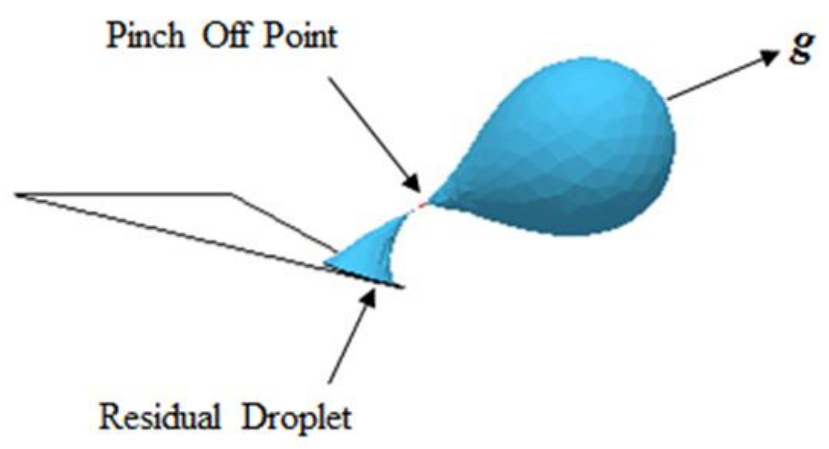

Figure 26. SE simulation showing the presence of residual fluid after droplet detachment. $g$ is shown for reference. $B o=2.7, \varphi=60^{\circ}$. 
Several such experiments were conducted with the blade $\alpha=60^{\circ}$, for volumes 14.5 , $15,16,25$, and $50 \mu \mathrm{L}$. After each detachment event, the $10 \mu \mathrm{L}$ syringe was inserted into the remaining fluid on the transparency and the plunger withdrawn until all the fluid was removed from the slide. Because of the partially wetting properties of silicone oil this could be accomplished with a high degree of precision as long as care is taken not to ingest air into the syringe during the process.

It was found that for each experimental drop volume, the Residual Drop was consistently the same volume. Additionally, the speed of the mount's rotation (up to $29.8^{\circ}$ s) appeared to play to no role in the volume of the Residual Drop. Figure 27 shows a linear relationship between the Bond number of the initial drops $\mathrm{Bo}_{\text {init }}$ against the Bond number of the residual drops $\mathrm{Bo}_{\text {res }}$.

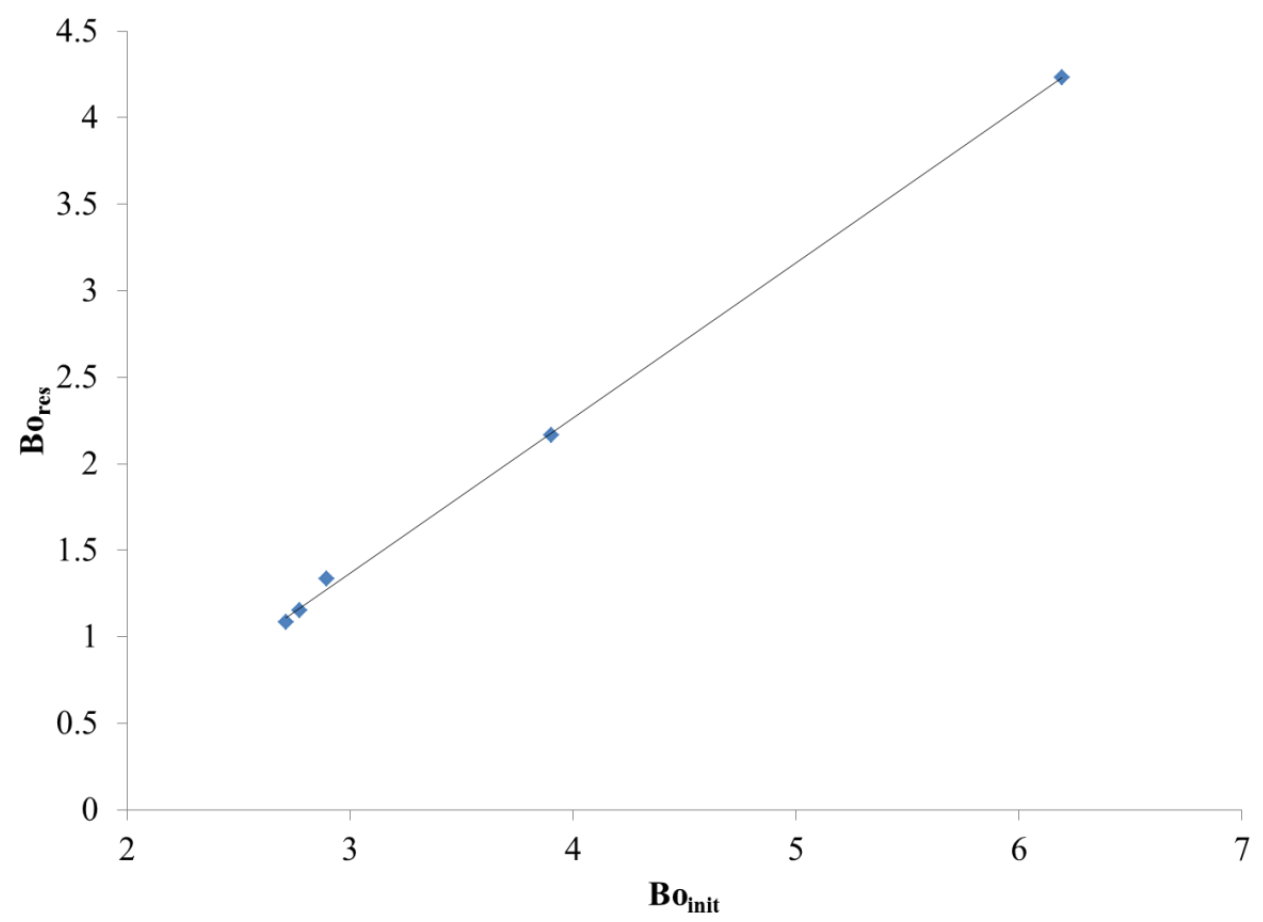

Figure 27. Initial drop Bond number $B o_{\text {init }}$ plotted against the residual drop Bond number $B o_{\text {res }} \cdot \mathbf{A}$ best fit regression line suggests a linear relationship with $R^{2} \approx 0.99$ and of the form $B o_{\text {res }}=0.896 B 0_{\text {init }}$ +1.32 . 
It should also be noted that for the range of $14.5-16 \mu \mathrm{L}$ the $\mathrm{Bo}_{\text {res }}$ increased at the same rate as $\mathrm{Bo}_{\text {init }}$ such that the volume of the detached drop was constant at $\sim 11 \mu \mathrm{L}$. This suggests that there could be several regimes for the volume of detached droplets which could provide valuable information for manufacturing processes where drop volume needs to be consistent.

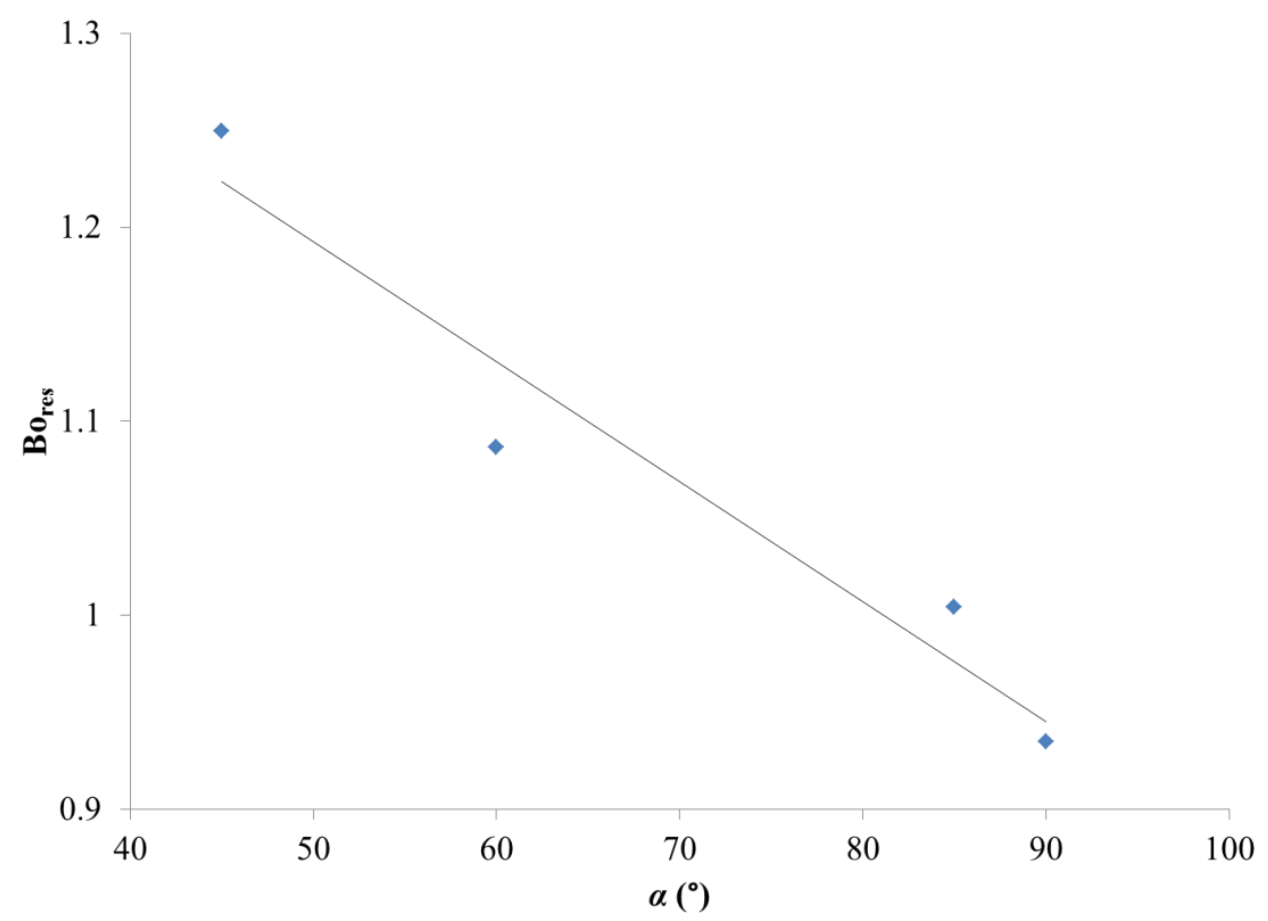

Figure 28. Plot of $\alpha$ versus the $B o_{\text {res. }}$ A best fit regression line suggests linear behavior with $\boldsymbol{R}^{2}=0.936$ of the form $B o_{\text {res }}=-6.2\left(10^{-3}\right) \alpha+1.5$.

Further experiments were performed to ascertain if there is an $\alpha$ dependence on the Residual Drop. The $14.5 \mu \mathrm{L}$ drop volume was again selected. The same procedure detailed above was repeated for half corner-angles $\alpha=45^{\circ}, 60^{\circ}, 85^{\circ}$, and $90^{\circ}$. Not only is the detached drop volume again seen to be constant within each $\alpha$, but an inverse linear relationship is seen between $\alpha$ and the $\mathrm{Bo}_{\text {res. }}$. This suggests that narrower blades, smaller $\alpha$, provide greater stability for a Wall-Edge-Vertex-Bound drop. The least stable is the 
extreme case of $\alpha=90^{\circ}$ or the edge of infinite length studied previously by Chen et al. [10]. Continued investigation of Residual Drops include a wider range of corner halfangles, initial drop volumes, contact angles, and wedge materials to determine what factors influence the volume of the Residual and Detached drops.

\subsection{Further Numerical Simulations $\left(\psi=0^{\circ}, \varphi=90^{\circ}\right)$}

The PSF was used to sweep a wide range of blade-bound drop geometric configurations. To simplify data reduction efforts ranges of geometric configurations were split between researches. The simulation range studied herein includes a $\boldsymbol{g}$-orientation of $\psi=0^{\circ}$ and $\varphi=$ $90^{\circ}$ while sweeping $\alpha$ and $\theta$.

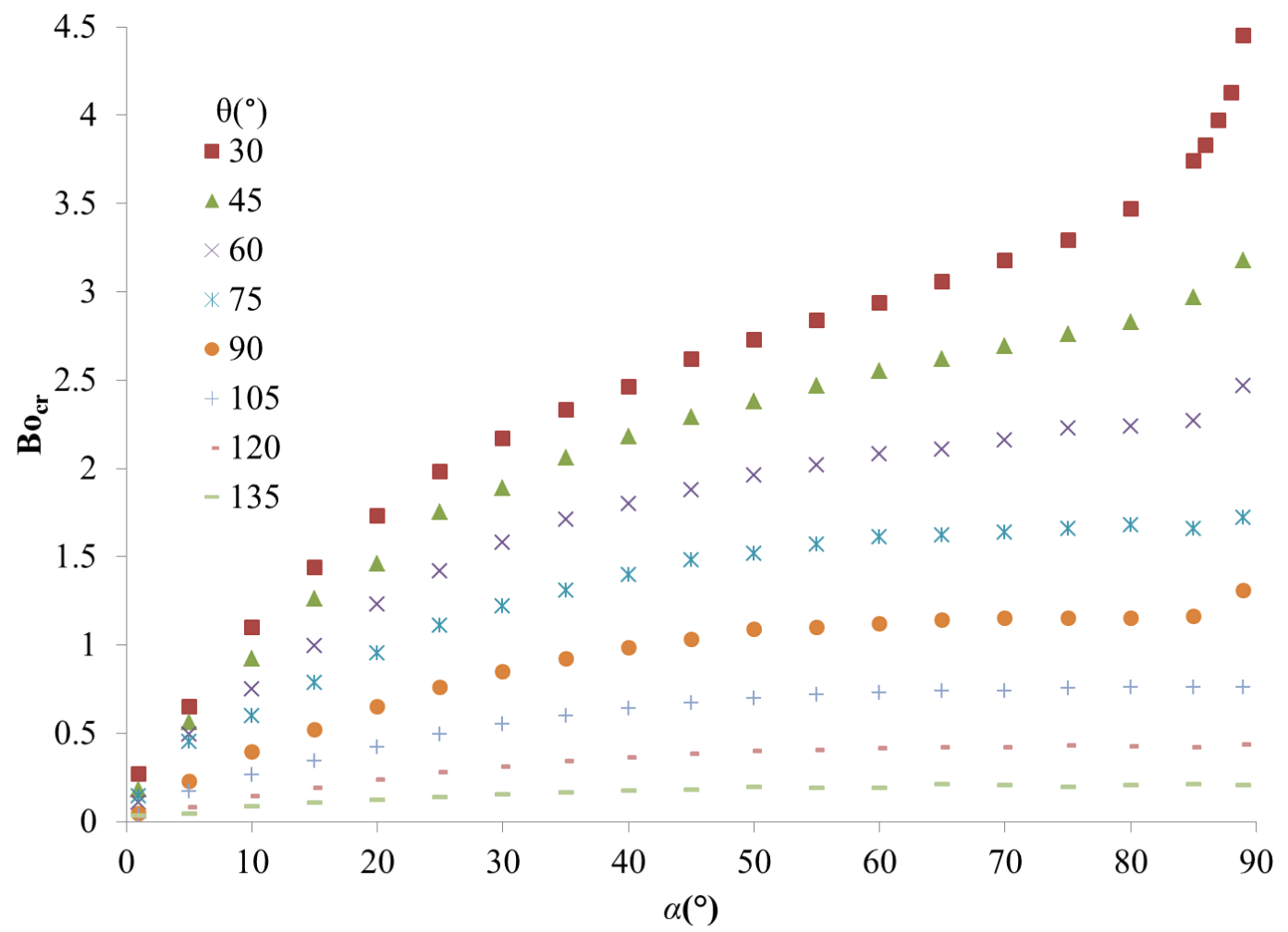

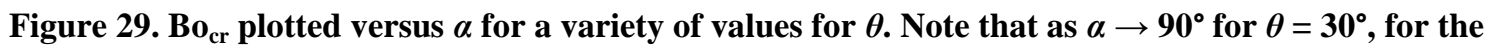
wall-edge-bound drop studied by Chen et al. [10], Bo $\rightarrow \infty$. 
Course and fine meshes were set to a maximal dimensionless length of 0.2 and 0.05 , respectively, with a total energy variation tolerance $<10^{-6}$ because as a droplet approaches the blade corner for small $\alpha$, the contact line requires more vertices to maintain accurate resolution. Simulations as those presented in Figure 29 were conducted for contact angles $\theta=30-135^{\circ}$ with sweeps of the corner half angle $\alpha=1$ through $89^{\circ}$.
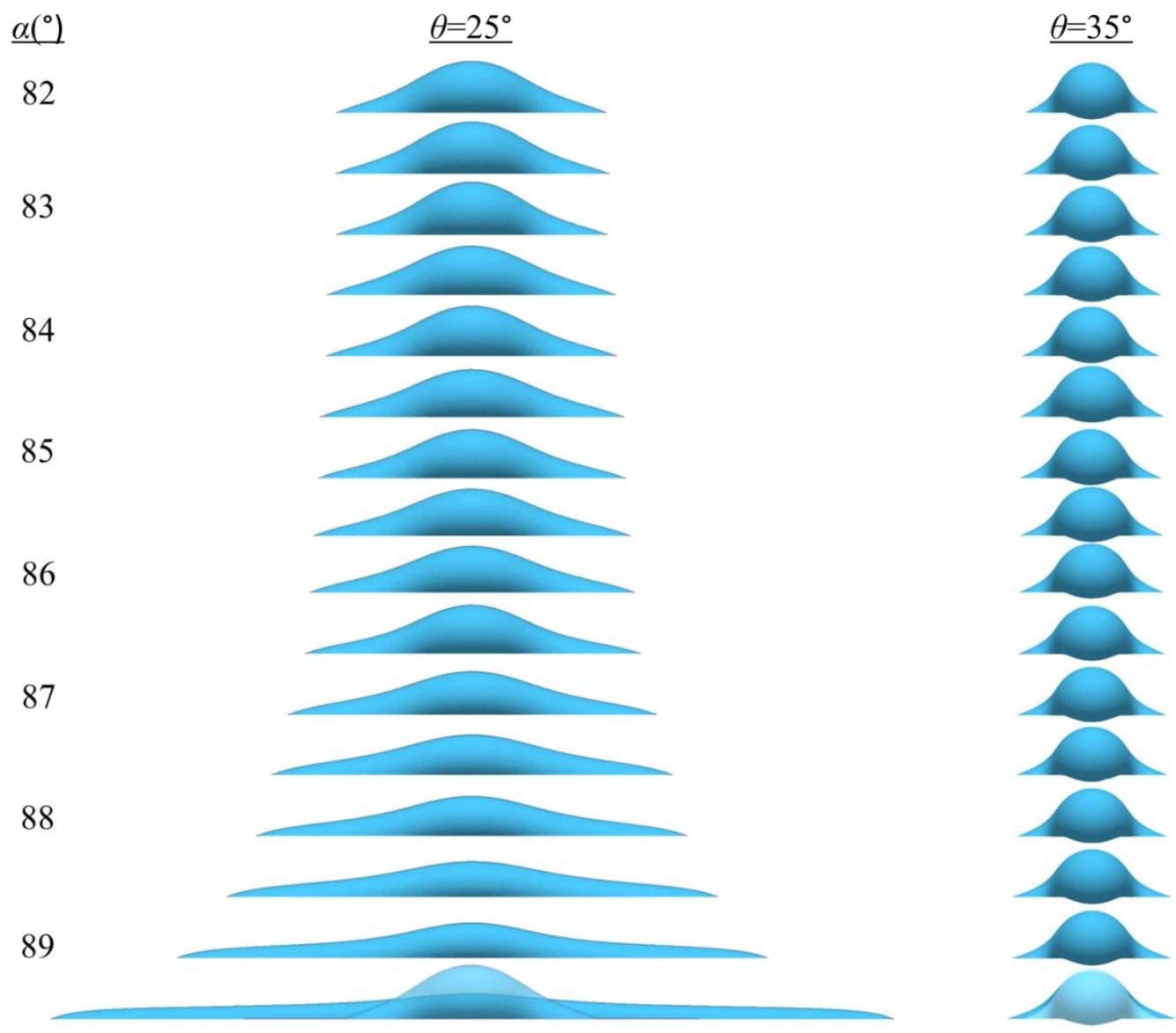

Figure 30. Point-on profile comparison of contact angles on either side of the $\theta=30^{\circ}$ Wall-EdgeBound drop condition. $\alpha$ is shown for a range of values. As $\alpha \rightarrow 90^{\circ}$, the $\theta=25^{\circ}$ case begins to spread to infinity while the $\theta=35^{\circ}$ case shows little change. Semi-transparent profiles of the $\alpha=82^{\circ}$ profile are overlayed on the $89.5^{\circ}$ profile for ease of comparison.

It was found in [10] that drops with $\theta \leq 30^{\circ}$ have no critical Bond number when $\alpha=90^{\circ}$ since it requires less energy to wet along the edge than to fall off. Simulations 
were conducted for $\theta=25^{\circ}$ and $35^{\circ}$ with corner half-angles approaching $90^{\circ}$ and the critical configuration profiles are compared in Figure 30.

The height and width of the $\theta=35^{\circ}$ profiles seen in Figure 30 shows almost no difference between $\alpha=82^{\circ}$ and $\alpha=89.5^{\circ}$, while $\theta=25^{\circ}$ shows a reduction in height and a radical expansion of the width. To add qualitative meaning to the profile visualization Figure 31 shows the $\mathrm{Bo}_{\mathrm{cr}}$ of each simulation. As expected, the minor changes in profile for $\theta=35^{\circ}$ translates into little variation in $\mathrm{Bo}_{\mathrm{cr}}$ while the opposite can be seen for $\theta=$ $25^{\circ}$ which shows $\mathrm{Bo}_{\mathrm{cr}} \rightarrow \infty$.

Investigations are ongoing into the study of edge-on profiles and the dependence of $\theta$ and $\alpha$ on the height and width of the drop at the critical configurations, which should yield insight into creating models for design purposes.

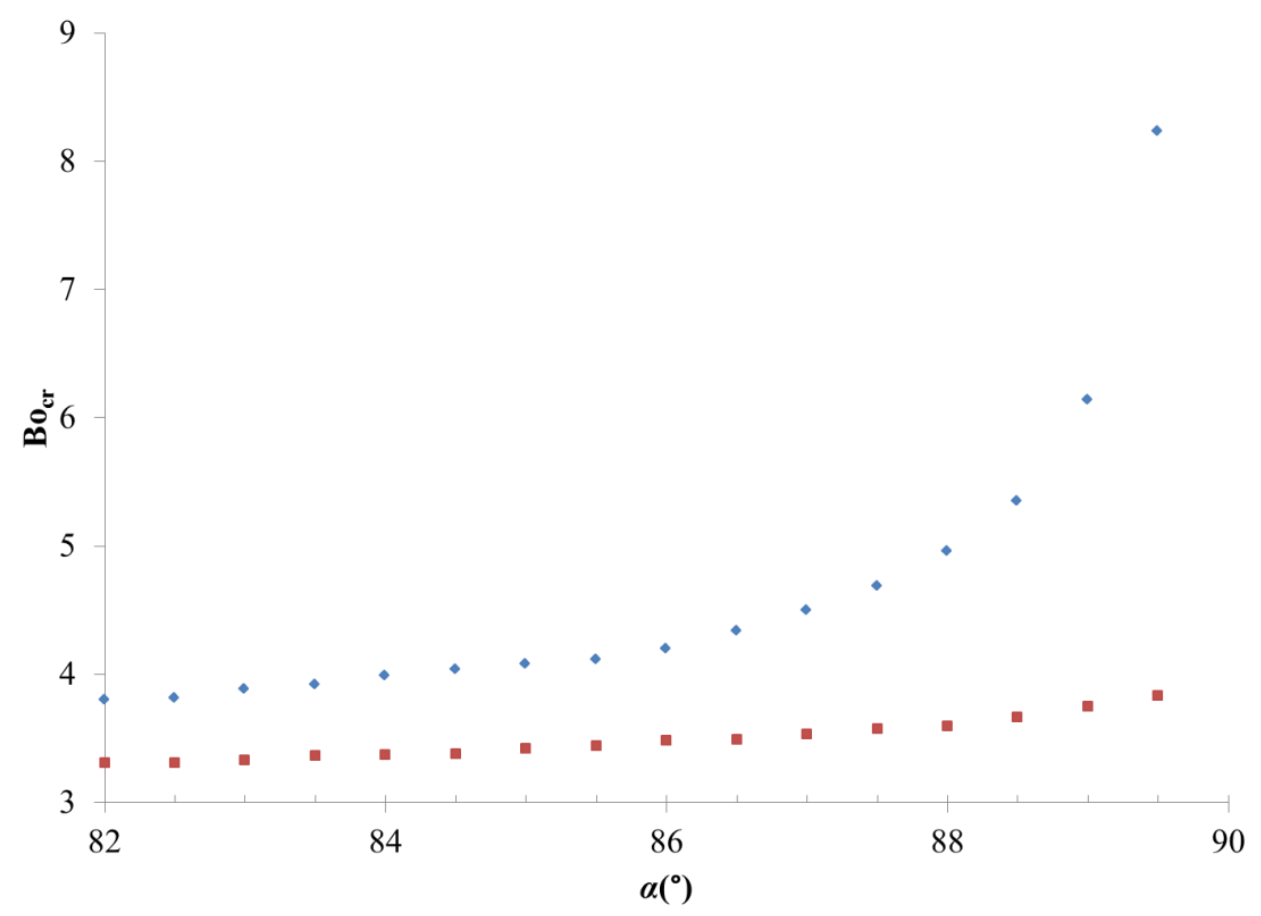

Figure 31. $\alpha$ plotted against $\mathrm{Bo}_{\mathrm{cr}}$ for $\theta=25^{\circ}$ and $\theta=35^{\circ} . \theta=25^{\circ}$ is seen approaching infinity, while $\theta=35^{\circ}$ has little relative variation. 
For example, current models are based on the more generalized case of the pendant drop, sketched in Figure 32. In order for the drop to detach from the surface the gravitational force needs to exceed the surface tension force holding it to the surface. The critical configuration is the last configuration when such a balance holds. Using a force balance we find that

$$
\rho V g=\operatorname{Sin}(\theta) F_{\sigma}
$$

where $\rho$ is the density difference between the gas and liquid, $V$ is the drop volume, $g$ is the magnitude of gravity, $\theta$ is the contact angle, and $F_{\sigma}$ is the magnitude of the surface tension force. Integrating around the contact line gives

$$
\rho V g=2 \pi r \sigma \operatorname{Sin}(\theta),
$$

where $r$ is the radius of the circle the contact line makes with the surface.

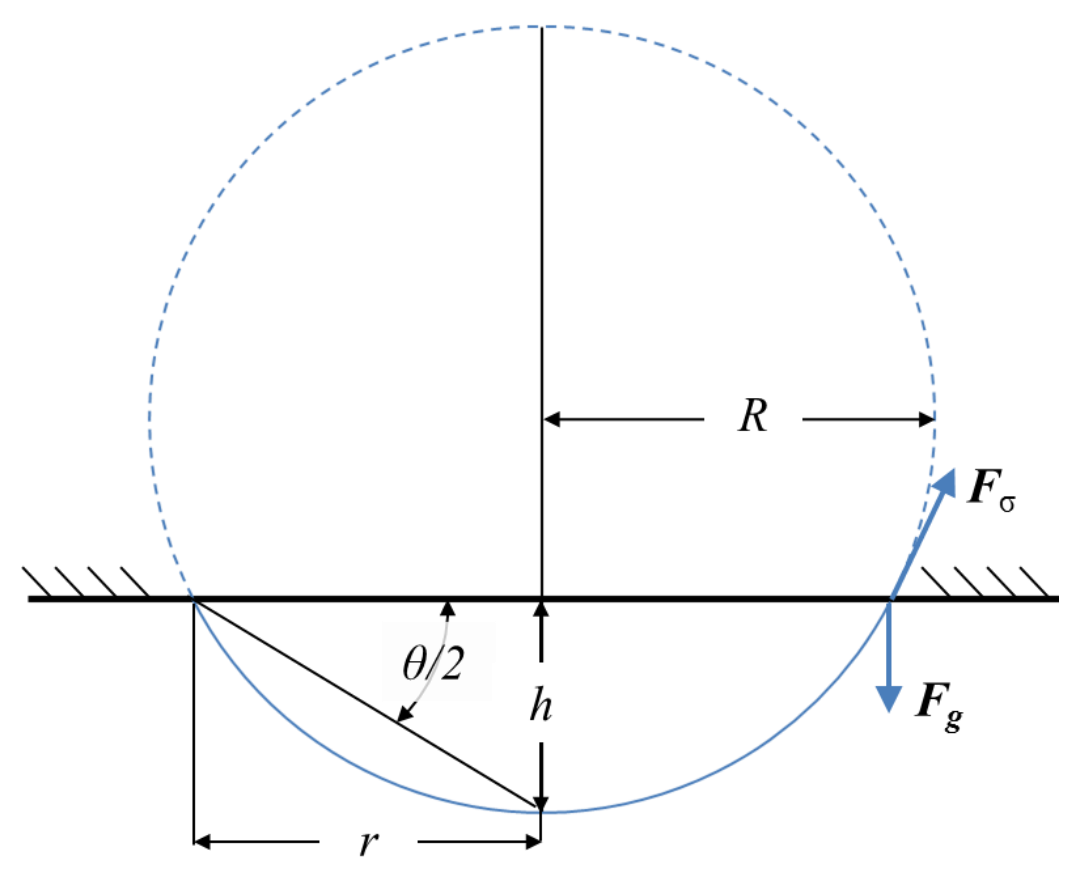

Figure 32. Sketch of a pendant drop with notation employed in the analysis. The dashed line is an extension of the droplet were the sphere to be extended into the plane. 
The volume of a spherical cap is defined as

$$
V=\frac{\pi h}{6}\left(3 r^{2}+h^{2}\right)
$$

with

$$
h=r \tan \left(\frac{\theta}{2}\right)
$$

Substituting Equations 4.3 and 4.4 back into Equation 4.2 yields

$$
\rho g\left[\frac{1}{6} \pi r^{2} \tan \left(\frac{\theta}{2}\right)\left(3 r+\tan \left(\frac{\theta}{2}\right)\right]=2 \pi r \sigma \sin (\theta)\right.
$$

which after rearranging and using the double angle formula for the sine function becomes

$$
1=\frac{1}{24} \frac{\rho g}{\sigma}\left[3 r+\tan \left(\frac{\theta}{2}\right)\right] \cos ^{-2} \frac{\theta}{2}
$$

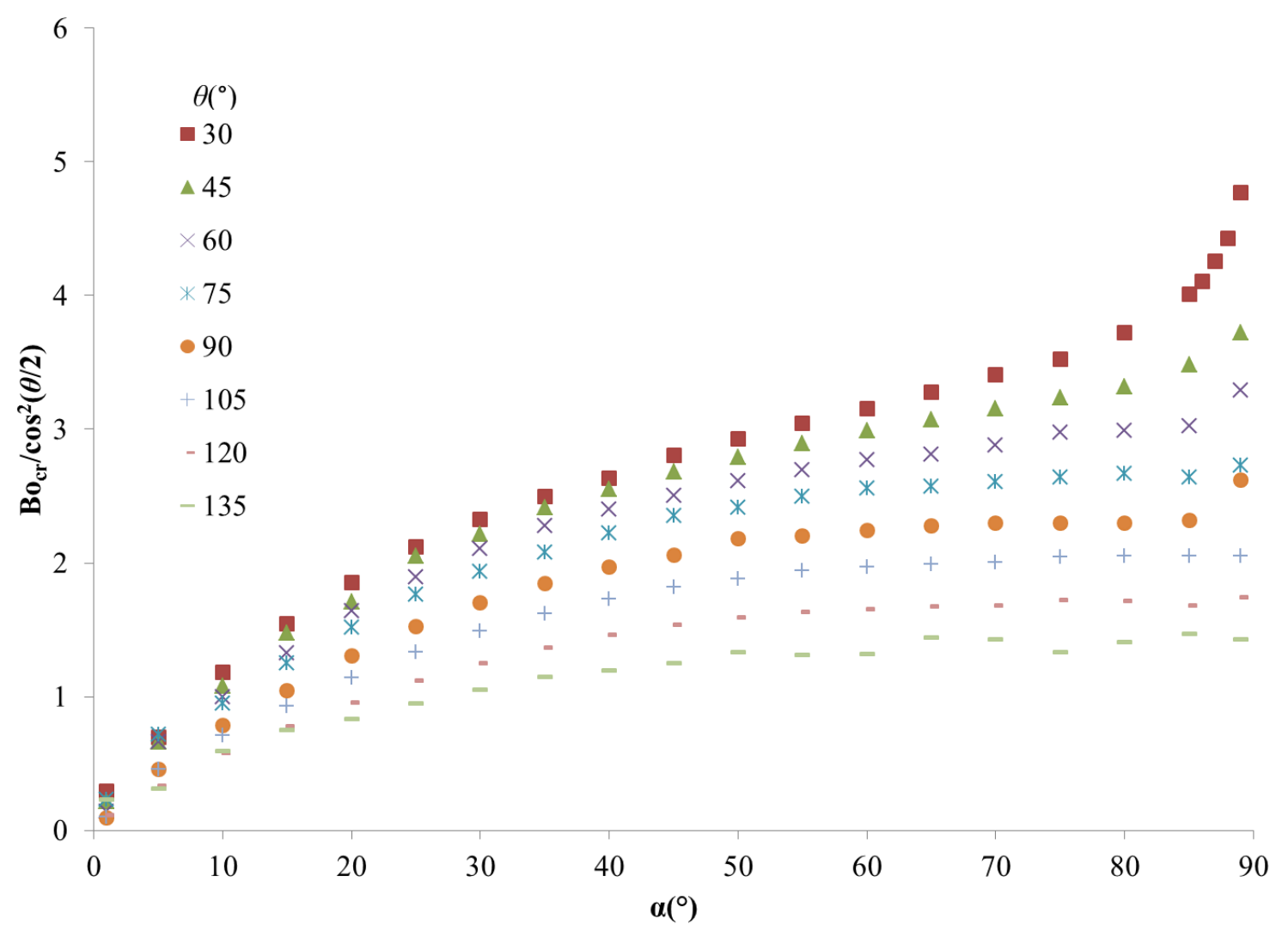

Figure 33. $\mathrm{Bo}_{\mathrm{cr}}$ data from Figure 29 after the initial correlation model is applied. The data partially collapses which encourages other modeling attempts along these lines. 
Equation 4.6 is certainly an approximation since it does not take into account $\alpha$ or $g$ orientation; however it does provide clues towards investigating a possible correlation model. Applying $1 / \cos ^{2}(\theta / 2)$ to the $\mathrm{Bo}_{\mathrm{cr}}$ in Figure 29 collapses a large portion of the data, though a dependence on $\alpha$ remains (see Figure 33).

After numerous other attempts a scaled critical Bond number

$$
B o_{c r}^{\prime}=\frac{\sqrt{\alpha}}{\sin (\alpha)} \cos ^{-3}\left(\frac{\theta}{2}\right) \sim O(1)
$$

was attempted. Figure 34 shows the collapsed data after the scaled critical Bond number of Equation 4.7 is applied. Much of the data collapses though there is still difficulties as $\alpha$ approaches the extremes at $0^{\circ}$ and $90^{\circ}$. It is likely that narrow and wide $\alpha$ blades operate under different regimes.

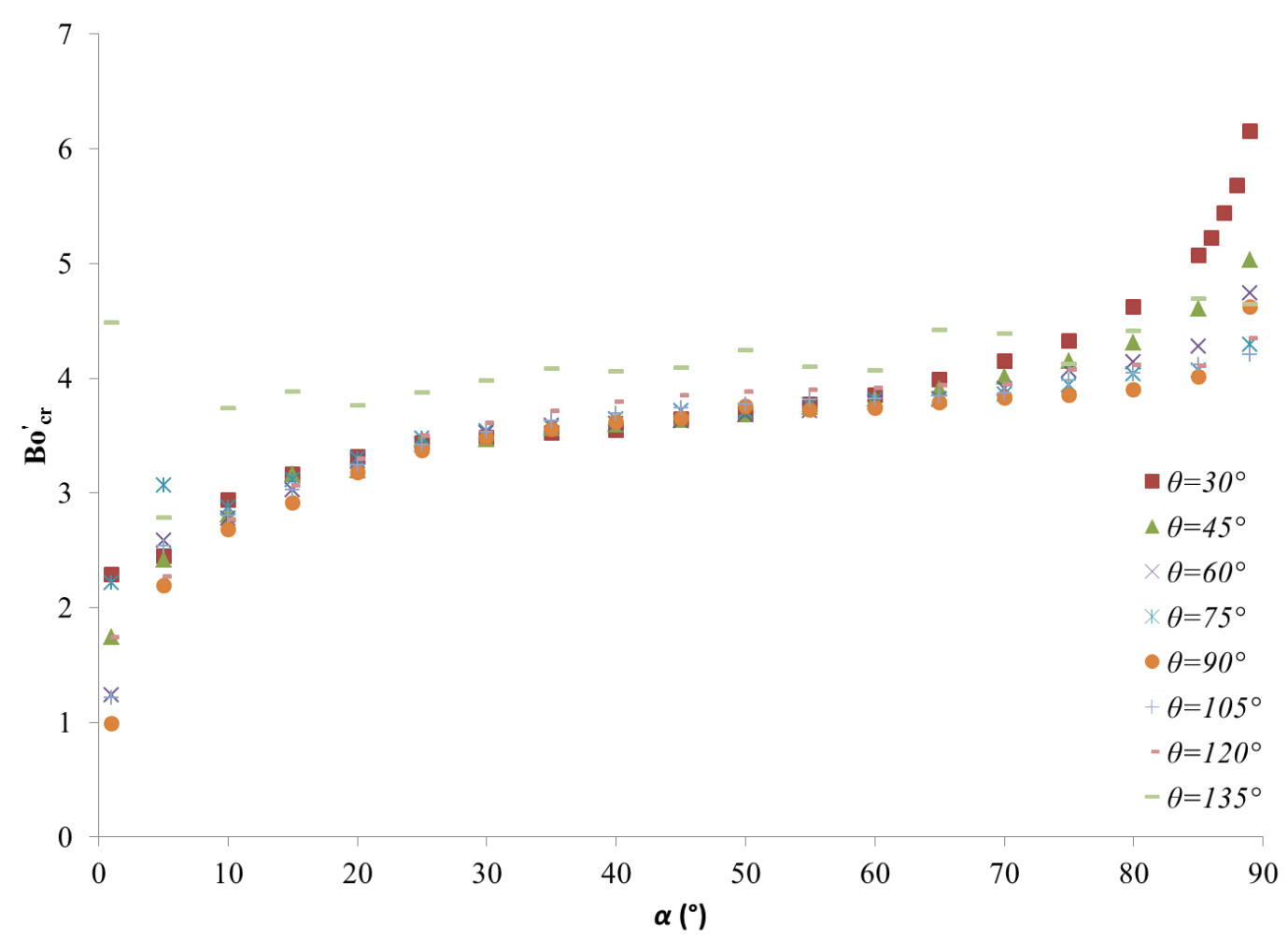

Figure 34. Bo' ${ }_{\text {cr }}$ plotted against $\alpha$. The data for mid-range $\alpha$ collapse together while different pinning regimes become apparent at the extremes. 

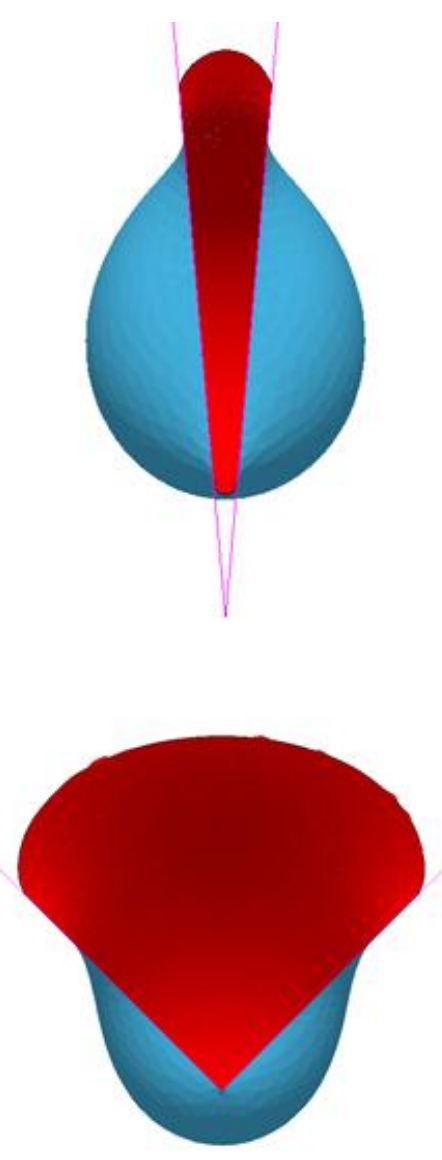

a.

Figure 35. Bottom view of $S E$-FIT simulations showing dry tips (above) at $\alpha=5^{\circ}$ and a wet tip (below) for $\alpha=45^{\circ}$ for a. $\theta=30^{\circ}$, b. $\theta=60^{\circ}$ and c. $\theta=90^{\circ}$. From this perspective, the ligher region surrouding the blade indicates the exterior surface of the drops while the darker region within the blade shows were the drop contacts the wedge. $g$ is in the plane of the paper and pointed down.

\subsubsection{Dry Leaf Tips}

Subsequent to our computations we learned that dry leaf tips are ubiquitous in nature. So much so that one does not typically give a second thought to them. Throughout the course of thousands of bound-droplet simulations a dry tip phenomenon began to emerge only under certain geometric configurations (see Figure 35). Hundreds of simulations have been conducted in pursuit of the geometric conditions that produce dry tips, but hundreds more are needed to establish a quantitative method to predict the phenomenon. 
Unfortunately, as the droplet approaches the tip of the wedge the number of vertices needed to accurately resolve the contact line increases, thus requiring a refinement of the simulation mesh that significantly increases computer run times. When completed, the results of this study will be included in a paper currently under preparation for publication [34].

\subsection{Conclusions and Direction}

The primary results of the stability of drops on blades study are as follows:

1. The SE-FIT PSF batch process is an accurate predictor of the shape and behavior of Wall-Edge-Vertex-Bound drops for $\alpha=60^{\circ}$ and $\theta=60^{\circ}$.

2. A linear relationship is observed between the initial drop volume and the volume of the residual for the drop volume range of $14.5 \mu \mathrm{L} \leq V \leq 16 \mu \mathrm{L}$.

3. A correlation model is discovered which collapses a large portion of the data for the $\psi=0^{\circ}$ and $\varphi=90^{\circ}$ cases.

4. Dry blade tips are discovered to occur only under certain geometric configurations.

Surface Evolver is demonstrated as an excellent tool in studying capillary systems. Combined with SE-FIT, a wieldy tool to predict the shape of Wall-Edge-Vertex-Bound drops is proven useful in the study of drop stability on systems such as blades, leaves and needles. SE-FIT continues to provide insight for experimenters leading to improved research efforts and efficiency, while highlighting previously uninvestigated natural phenomenon. 
Highly resolved computations are currently underway to capture contact line geometry and pinning for blades with $\alpha \rightarrow 0^{\circ}$ and $\alpha \rightarrow 90^{\circ}$. Greater resolution at these extremes will provide insight into the geometric conditions that create phenomena such as dry leaf tips and the $\theta \leq 30^{\circ}$ infinite spread condition for Wall-Edge-Bound drops. Additionally, thousands of simulations performed by the other graduate student researchers previously mentioned will be combined to create a correlation model of greater brevity than the simple pendant case model investigated herein.

Utilizing the ease with which SE-FIT allows the user to create and run simulations on new geometries, additional complexity could be added to the existing Wall-Edge-VertexBound drop investigations by introducing a creasing angle. So called Corner-EdgeVertex-Bound constraints are more reminiscent of a spade as opposed to the blades studied herein. As with Blade-Bound droplets, knowledge of Spade-Bound droplet stability may be critical to the performance of numerous systems aboard spacecraft [13], and Earth-based biological systems and industrial processes. 


\section{References}

1 Kirby, B.J., Micro- and Nanoscale Fluid Mechanics: Transport in Microfluidic Devices, Cambridge University Press, New York, 2010.

2 Yager, P., Edwards, T., Fu, E., Helton, K., Nelson, K., Tam, M.R., and Weigl, B.H., "Microfluidic diagnostic technologies for global public health." Nature, Vol. 442, No. 7101, July 2006, pp. 412-418.

3 Blackmore, W., Weislogel, M.M., Chen, Y., Bunnell, C.T., Kiewidt, L., and Klatte, J., "The Capillary Flow Experiments (CFE-2) on ISS: Status," 49th AIAA Aerospace Sciences Meeting, AIAA, Orlando, Florida, 4-7 January 2011, 10 pages.

4 Jenson, R.M., Weislogel, M.M., Chen, Y., Tavan, N.T., and Bunnell, C.T., "The Capillary Flow Experiments aboard the International Space Station: Increments 9-15, August 2004 to December 2007," NASA/CR—2009-215586, September 2009.

5 "SpaceX CRS-1 Mission" (Press release). NASA. Available at: http://www.nasa.gov/pdf/694074main_SpaceXCRS-1PressKit.pdf , October 2012, Acessed 10/8/2012.

6 Salim, A., Colin, C., and Dreyer, M. E., "Experimental Investigation of a Bubbly Two Phase Flow in an Open Capillary Channel under Microgravity Conditions," J. Microgravity Sci. Technol., Vol. 22, 2010, pp. 87-96.

7 Canfield, P.J., Bronowicki, P.M., Chen, Y., Kiewidt, L., Dreyer, M.E., Grah, A., Klatte, J., Jenson, R., Blackmore, W., and Weislogel, M.M., "The Capillary Channel Flow Experiments on the International Space Station: Experiment Setup and First Results," Experiments in Fluids, June 2012 (in preparation).

8 Blackmore, W., Weislogel, M.M., Balasubramaniam, R., and Kelly, E., "Unresolved Low-g Bubble Migration Phenomenon: Peripheral Observations during Fluids Experiments aboard the ISS," 50th AIAA Aerospace Sciences Meeting, AIAA, Nashville, Tennessee, 9-12 January 2012, 11 pages.

9 “EVA Shortened by Faulty Sensor," YouTube, NASAtelevision, 20 May 2011, Web, Accessed 10/8/2012.

10 Chen, Y., and Bacich, M.A. et al., "The Shape and Stability of Wall-Bound and Wall-Edge-Bound Drops and Bubbles," J. Microgravity Sci. Technol., Vol. XVII, No. 4, 2005, pp. 14-24. 
11 Brakke, K.A., “The Surface Evolver," Experimental Mathematics, Vol. 1, 1992, p.p. 141-165.

12 Chen, Y., Surface Evolver - Fluid Interface Tool, 2012, Available for download at: http://www.se-fit.com, Accessed 10/1/2012.

13 Weislogel, M.M., Thomas, E.A., and Graf, J.C., "A Novel Device Addressing Design Challenges for Passive Fluid Phase Separations Aboard Spacecraft," J. Microgravity Sci. Technol., August 06, 2008, 12 pages.

14 Concus, P., and Finn, R., "On the Behavior of a Capillary Free Surface in a Wedge," Proc. Nat. Acad. Sci. U.S.A., Vol. 63, No. 2, June 1969, pp. 292-299.

15 Chen, Y., R. Jenson, M. Weislogel, and S. Collicott, "Capillary Wetting Analysis of the CFE-Vane Gap Geometry," 46th AIAA Aerospace Sci. Meeting and Exhibit, AIAA, 7-10, January 2008.

16 Skinner, C.A., Gershman, R., and Kvingge, L.C., "Space Simulation Testing for the Skylab Waste Tank," McDonnell Douglas Astronautics Co., 74N-10233, Paper No. 1, 1973, pp. 1-6.

17 Jaekle, D.E., Jr., "Propellant Management Device Conceptual design and Analysis: Vanes," 27th AIAA/SAE/ASME/ASEE Joint Propulsion Conference, AIAA, 24-26 June 1991.

18 Dyson, D.C., "Contact line stability at edges: Comments on Gibbs's inequalities," Physics of Fluids, Vol. 31, No. 2, February 1995, pp. 229-232.

19 Paterson, A., Fermigier, M., Jenffer, P., and Limat, L., "Wetting on heterogeneous surfaces: experiments in an imperfect Hele-Shaw cell," Physical Review E, Vol. 51, No. 2, February 1995, pp. 1291-1298.

20 Anantharaju, N., Panchagnula, M.V., Vedantam, S., Neti, S., and Tatic-Lucic, S., "Effect of Three-Phase Contact Line Topology on Dynamic Contact Angles on Heterogeneous Surfaces," Langmuir, Vol. 23, 2007, pp. 11673-11676.

21 Chen, Y., N. Tavan, and M. M. Weislogel., "A Mean Curvature Model for Capillary Flows in Asymmetric Containers and Conduits," Physics of Fluids, Vol. 12, American Institute of Physics, 8/31/2012, Web, Accessed 10/1/2012.

22 Chowdhury, S. Bhethanabotla, V. R., and Sen, R., "Measurement of Diffusion Coefficient of Oxygen in FRP Using Luminescence Quenching," Composites \& Polycon, 17-19 October 2007. 
23 Subramanian, R., and Balasubramaniam, R., The Motion of Bubbles and Drops in Reduced Gravity, Cambridge University Press, New York, 2001, pp. 57.

24 OpenCFD, OpenFOAM: The open source CFD toolbox - User Guide Version 1.6. 2009. Available for download at: http://www.openfoam.org

25 Weller, H. G., "Derivation, modelling and solution of the conditionally averaged two-phase flow equations," Technical report, OpenCFD Ltd., United Kingdom, 23 February 2005.

26 Brenner, H., "Effect of finite boundaries on the Stokes resistance of an arbitrary particle," Journal of Fluid Mechanics, Vol. 12, 1962, pp. 35-48.

27 Ostrach, S., "Low-Gravity Fluid Flows," Annual Review of Fluid Mechanics, Vol. 14, 1982, pp. 313-345.

28 Young, N. O., Goldstein, J. S., and Block, M. J., "The motion of bubbles in a vertical temperature gradient," Journal of Fluid Mechanics, Vol. 6, No. 03, 1959, pp. 350-356.

29 Hardy, S.C., "The motion of bubbles in a vertical temperature gradient," Journal of Colloid and Interface Science, Vol. 69, No. 1, 15 March 1979, pp. 157-162.

30 Rednikov, A.Ye., and Ryazantsev, Yu.S., "Thermocapillary motion of a droplet heated by radiation," International Journal of Heat and Mass Transfer, Vol. 35, No. 1, January 1992, pp. 255-261.

31 Ryazantsev, Yu., and S Rednikov, A. Ye., "The influence of Heat Generation in a Droplet on Thermocapillary Force," 17th International Symposium on Space Technology and Science, Tokyo, Japan, Vol. 2, 1990, pp. 2159-2164.

32 Rednikov, A.Ye., and Ryazantsev, Yu.S., "On the thermocapillary motion of a drop with homogeneous internal heat evolution," Journal of Applied Mathematics and Mechanics, Vol. 53, No. 2, pp. 212-216.

33 DeWitt, D.P., and Incropera, F. P., Computational Methods in Fluid Flow, 5th ed., Wiley, New York, 2002, pp. 257.

34 Chen, Y., Hinojosa, C., Wollman, A., Blackmore, W., Jenson, R., Nguyen, D., and Weislogel, M.M., "Drops on Leaves," Physics of Fluids, (in preparation) 2013. 
35 Klimek, R., and Wright, T., Spotlight-16: Image analysis and object tracking software, Available for download at: http://exploration.grc.nasa.gov/spotlight/, 2004.

36 Weislogel, M.M., Jenson, R., and Bolleddula, D., "Capillary Driven Flows in Weakly 3-Dimensional Polygonal Containers", 45th AIAA Aerospace Sciences Meeting and Exhibit, AIAA, Reno, Nevada, 8-11 January 2007.

37 Weislogel, M.M., Baker, J. A., and Jenson, R. M., "Quasi-steady capillary-driven flows in slender containers with interior edges," J. Fluid Mech., Vol. 685, 2011, pp. 271-305.

38 Canopus System, Inc., "OARE Technical Report 149, STS-78 (LMS-1) Final Report,” CSI-9604, September 1996.

39 Canopus System, Inc., "OARE Technical Report 151, STS-94 (MSL-1) Final Report," CSI-9704, August 1997.

40 Hogg, Robert V., "Adaptive Robust Procedures: A partial Review and some Suggestions for Future Applications and Theory," Journal of the American Statistical Association, Vol. 69, December 1974 .

41 Matisak, B.P., Rogers, M.J.B, and Alexander J.I.D., "Analysis of the Passive Accelerometer System (PAS) Measurements During USML-1," AIAA-94 0434, December 1994.

42 Jules, K., Hrovat, K., Kelly, E., McPherson, K., and Reckart, T., "International Space Station Increment 6/8 Microgravity Summary Report," NASA/TM-2005213896, July 2005. 


\section{Appendix A}

\section{List of CFE-2 Experiments Performed to Date}

1. ICF1-3 (T. Caldwell-Dyson): Pre-Wetted and Wetted 30ml tests

2. ICF1-4 (S. Walker): Remaining 30, 25, and 20ml tests and 30, 25, and 20ml Bubble Shake tests

3. ICF1-5 (S. Walker): Additional 30ml test and 20-25ml bubble shake tests

4. ICF1-6 (S. Kelly): Initial investigation into transport tube bubble migration phenomenon

5. ICF1-7 (C. Coleman): Bonus Bubble Shake test for C. Coleman interview on The Talk

6. ICF1-8 (D. Burbank): Compressed Bubble Test 2

7. ICF2-3 (S. Kelly): Pre-Wetted 25 and several Wetted $25 \mathrm{ml}$ runs performed

8. ICF2-4 (S. Kelly): Completion of 20 and $15 \mathrm{ml}$ test points

9. ICF2-5 (C. Coleman): Bubble Shake Test

10. ICF2-6 (R. Garan): Compressed Bubble Migration Test

11. VG1-6 (S. Kelly): Completion of Pre-Wetted CW rotation test

12. VG1-7 (S. Kelly): Completion of Wetted CW rotation test

13. VG1-8 (S. Kelly): Completion of CCW rotation test

14. VG1-9 (S. Kelly): Completion of CW/CCW rotation through quadrants Q3 and Q4

15. VG1-10 (S. Kelly): Q1/Q2 Test

16. VG1-11 (M. Fossum): Filled Perforations CW Q1/Q2 Test 
17. VG1-12 (M. Fossum): Filled Perforations CW Q3/Q4 Test

18. VG1-13 (D. Burbank): Fillled Perforations Q1 Test

19. VG1-14 (D. Burbank): Filled Perforations Q2 Test

20. VG1-15 (D. Pettit): Filled Perforations Q3 Test

21. VG1-16 (D. Pettit): Filled Perforations Q4 Test

22. VG2-5 (M. Fossum): CW Dry Test

23. VG2-6 (D. Pettit): CW Wet Test

24. VG2-7 (D. Pettit): CCW Wet Test

25. VG2-8 (D. Burbank): Q1 Test

26. VG2-9 (D. Burbank): Q2 Test

27. VG2-10 (D. Pettit): Q3 Test

28. VG2-11 (J. Acaba): Q4 Test

29. VG2-12 (J. Acaba): Filled Perforations CW Test

30. VG2-13 (J. Acaba): Filled Perforations CCW Test 


\section{Appendix B}

\section{CFE-2 ICF Experiments}

\section{The Interior Corner Flow Experiments (ICF1 through ICF9)}

The ICF experiment seeks to at least determine: (1) the rates of 3-D imbibition of wetting

fluids in containers of systematically increasing complexity, (2) the dependence of model dynamical boundary conditions as a function of geometry, and (3) the performance of such devices as passive phase separators (i.e. converting bubbly flows to separated continuous gas and liquid inventories). On a local level, the initial wetting rates of such flows are fast and can be studied using drop towers. However, a slow migration ('secondary imbibition') of fluid across the chamber driven by the combined effects of capillary forces and global changes in container dimensions at present can only be thoroughly and convincingly studied in the long duration low-g environment of the ISS.

The test cells employ characteristic dimensions of current spacecraft equipmentapproximately two orders of magnitude larger than similar systems on Earth. This choice significantly alters the time scales of the flow and dramatically increases $\left(10^{4}\right.$-fold $)$ the volume of fluid involved as well as the effective accuracy of the test cell geometry. The experiments are designed to benchmark the analytical techniques developed to predict such flows. The benchmarked theory can then be used to design and analyze capillary devices for positioning liquids passively in containers in low-g environments by careful control over container geometry. The devices are used to perform passive phase separation operations as in the case of tapered screen galleries for bubble-free collection and positioning of fuels for satellites, an important and outstanding problem for 
propellant, coolant, or water management aboard spacecraft [11]. The ICF1 and ICF2 units were originally part of the CFE-1 project and were re-flown to ISS with increases in fluid viscosity and fluid volume. Schematics of the ICF test cells are provided in Figure 36 and Figure 37 with ICF1 and ICF2 details emphasized in Figure 36.

The objectives of the ICF tests include the experimental determination of secondary imbibition rates in complex containers due to spontaneous large length scale capillary flows along interior corners in weakly three-dimensional containers. Additional objectives include the identification of the correct dynamical boundary conditions for such flows as well as the quantification of passive phase separation characteristics of certain capillary driven multiphase-flows along interior corners. The experimental results will be used to verify or further guide current theoretical predictions. They will also provide benchmarks for numerical methods.

The 'taper' (or porosity gradient) of these containers provides particular design advantages in preferentially locating the liquid where desirable. The most current review of the present work may be found in Weislogel et al. [36,37]. Vessels ICF1 and ICF2 investigate parabolic and linearly tapered sections, respectively. The important ICF1 and ICF2 vessel properties are listed below:

\section{ICF1 (ref. Figure 36)}

1. Test cell: parabolically tapered 75-75-30 isosceles tri.

2. Height of vertex at base $4 \mathrm{~cm}$

3 . Height of vertex at top $2.6 \mathrm{~cm}$

4. Base of triangle and acute vertex taper at $3.155^{\circ}$

5 . Test cell is $12.7 \mathrm{~cm}$ tall

6. Fluid: $20 \mathrm{cs} \mathrm{Si}$ oil

7. Initial fluid volume is $30 \mathrm{ml}$

8. Bubble generation by shaking container 
ICF2 (ref. Figure 36)

1. Test cell: linearly tapered rectangular section

2. Only the side faces tilt at $8.95^{\circ}$

3 . Test cell is $12.7 \mathrm{~cm}$ tall and $4 \mathrm{~cm}$ wide at base

4. Test cell is a constant $1 \mathrm{~cm}$ deep

5. Fluid: 2cs Si oil

6. Initial fluid volume is $25 \mathrm{ml}$

7. Bubble generation by shaking container

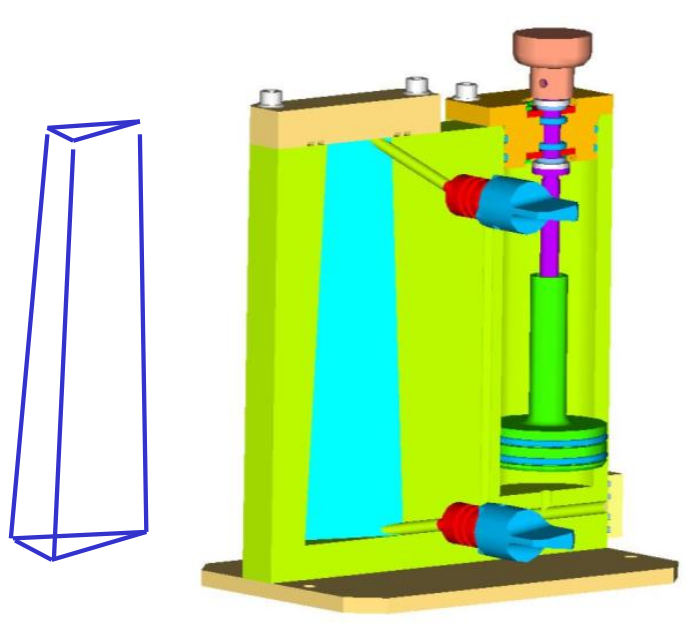

ICF1

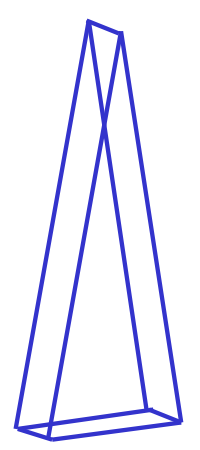

ICF2

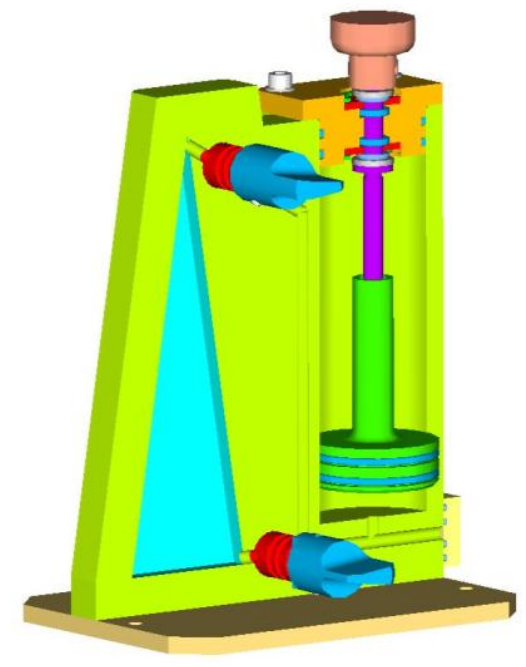

Figure 36. Wire and Solid models of ICF1 (truncated, pyramidally tapered 75-75-30 isosceles triangle) and ICF2 (linearly tapered rectangular section).

Several operations have been performed to date on both ICF1 and ICF2. In both containers a variable volume of fluid is pumped into the test containers. The fluid then wicks along the interior edges of the container and upon reaching the top forms another meniscus. Due to the tapered geometry of the containers the curvature of the newly created meniscus is higher than that of the meniscus at the bottom of the container. This establishes a pressure gradient in the fluid along the edges which continues to act to drive the liquid to the top of the container displacing the ullage to the bottom as shown in Figure 38 for the ICF1 30ml test. 


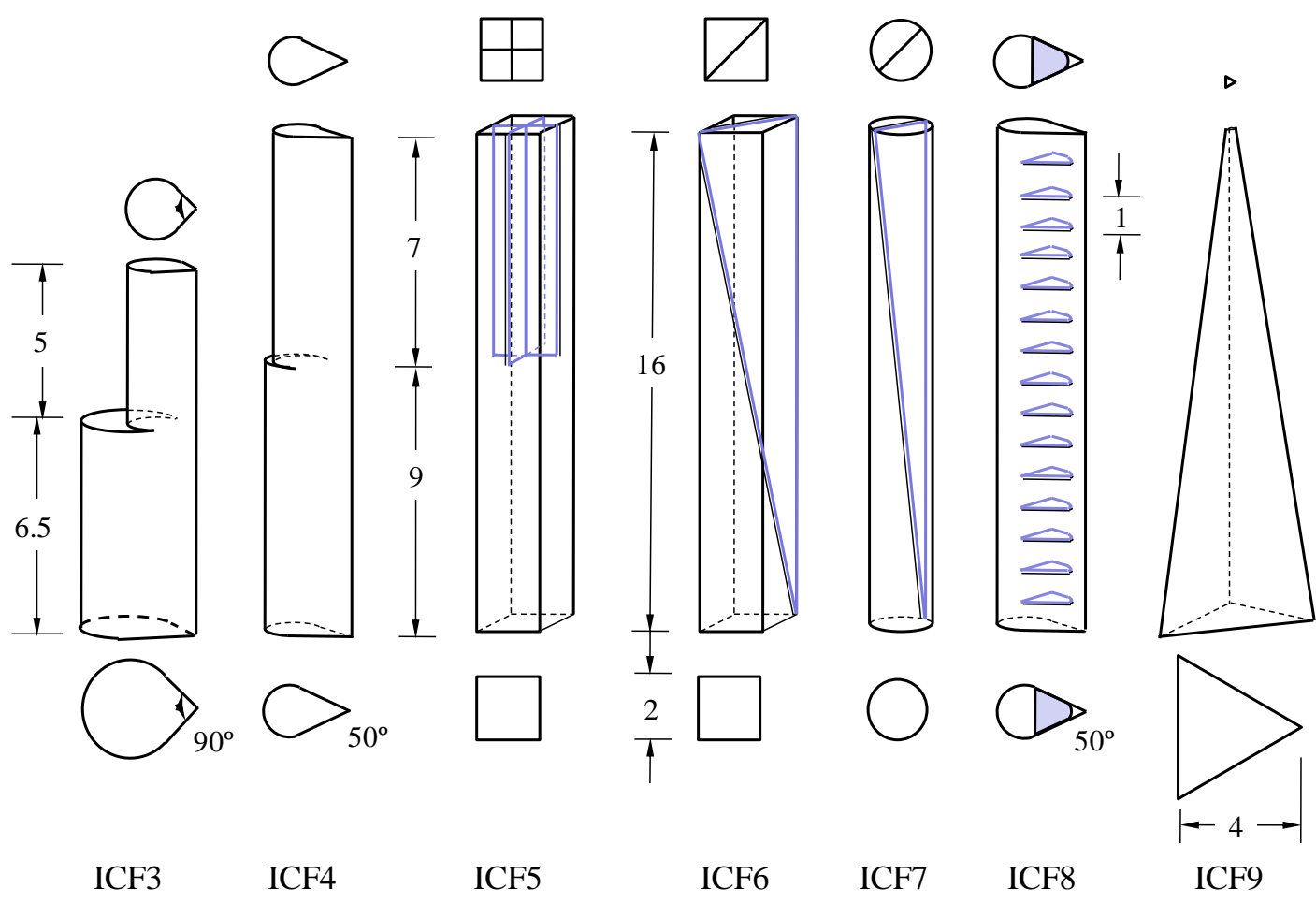

Figure 37. Schematics of CFE-2 ICF containers ICF3 through ICF9 (to scale). ICF3; stepped taper of $90^{\circ}$ 'snow cone' section, ICF4; stepped taper of $50^{\circ}$ snow cone section, ICF5; 'single-to-4-pack' square graded porous structure, ICF6; diagonal linearly tapered vane in square cylindrical section, ICF7; linearly tapered vane in right circular section, ICF8; segmented $50^{\circ}$ snow cone section, ICF9; complete parabolic taper of 60-60-60 triangular section (dimensions in $\mathrm{cm}$ ).
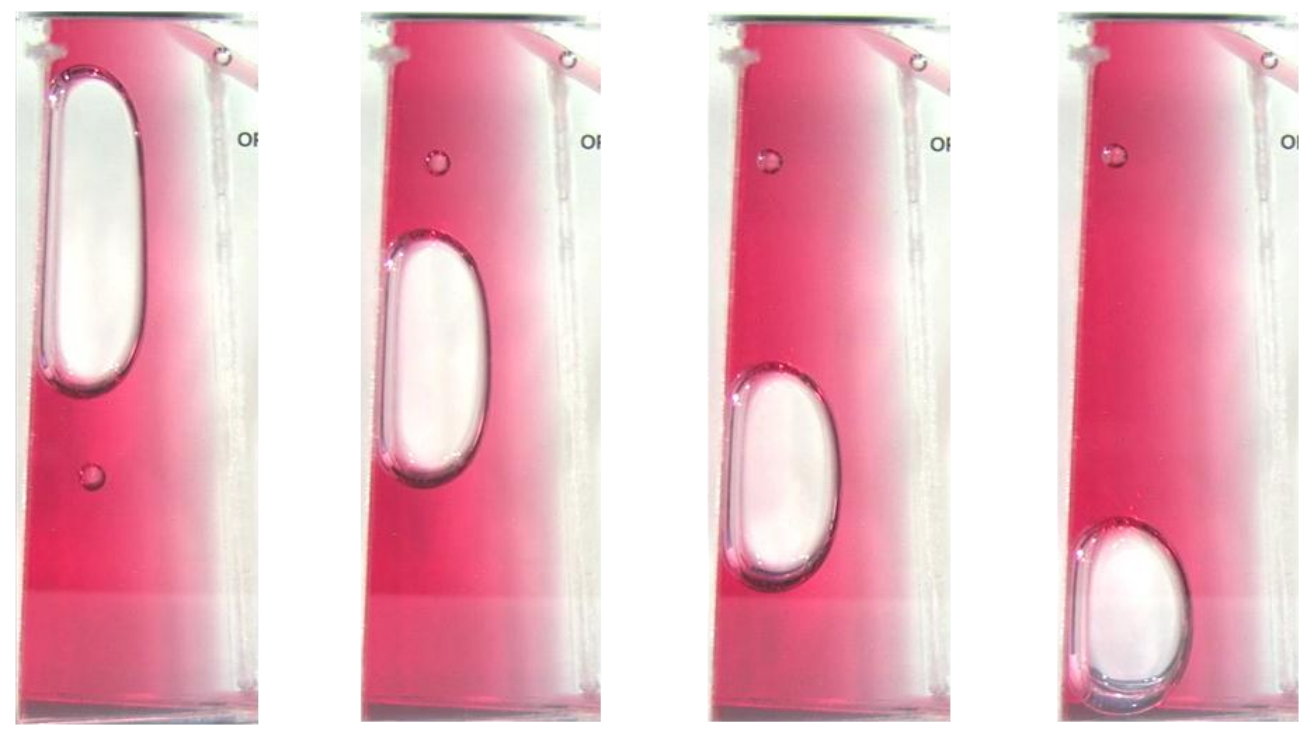

Figure 38. ICF1 30ml test: image sequence at 2.5 minute intervals. The ullage is displaced 'down' the container as fluid flows along the corners. 
The fluid volume is adjustable and experiments repeatable. For example, ICF1 tests are performed under Wetted and Pre-Wetted conditions at approximately 30, 25, and $20 \mathrm{ml}$. Dry surface tests are only conducted for the first run of the vessel for the $30 \mathrm{ml}$ fill condition shown in Figure 38. For each fluid volume, Bubble Shake tests are performed where the fluid is pumped into the test chamber and is then manually shaken by the astronaut. This causes the ullage to break up into bubbles of various sizes as shown in Figure 39. The migration of the fluid in the container continues to occur in a similar manner to that described above except that varying migration speeds for ullages of different sizes lead to coalescence events. ICF2 has also been operated under Wetted and Pre-wetted conditions with approximately 25,20 , and $15 \mathrm{ml}$ fluid volumes as shown in Figure 40 (25ml case).
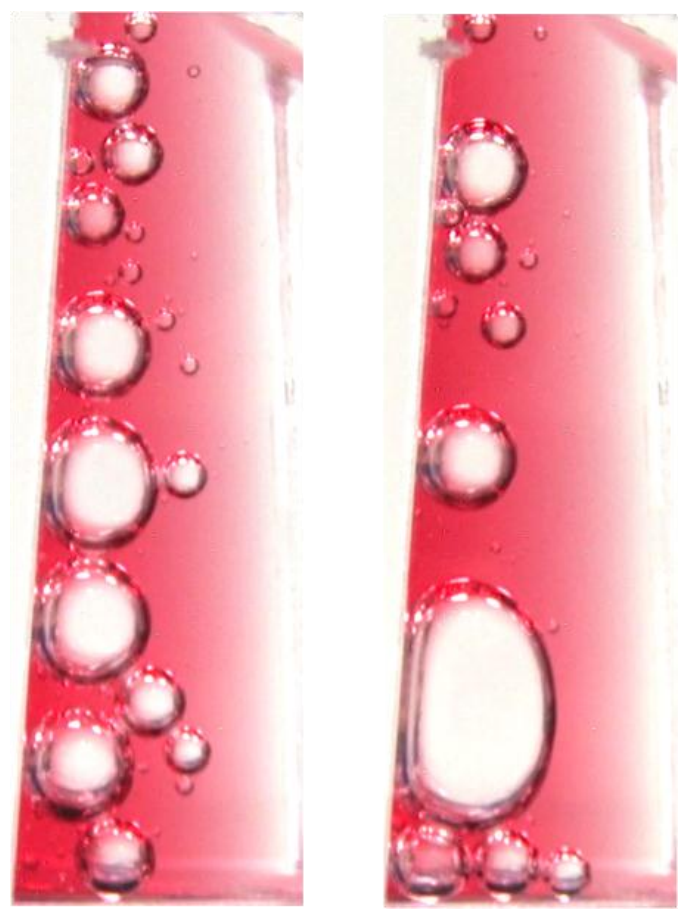

Figure 39. ICF1 30ml bubble shake test: the ullage is broken into several smaller bubbles (left) and as normal flow continues, coalescence continues to occur over a period of 3 minutes (right). 

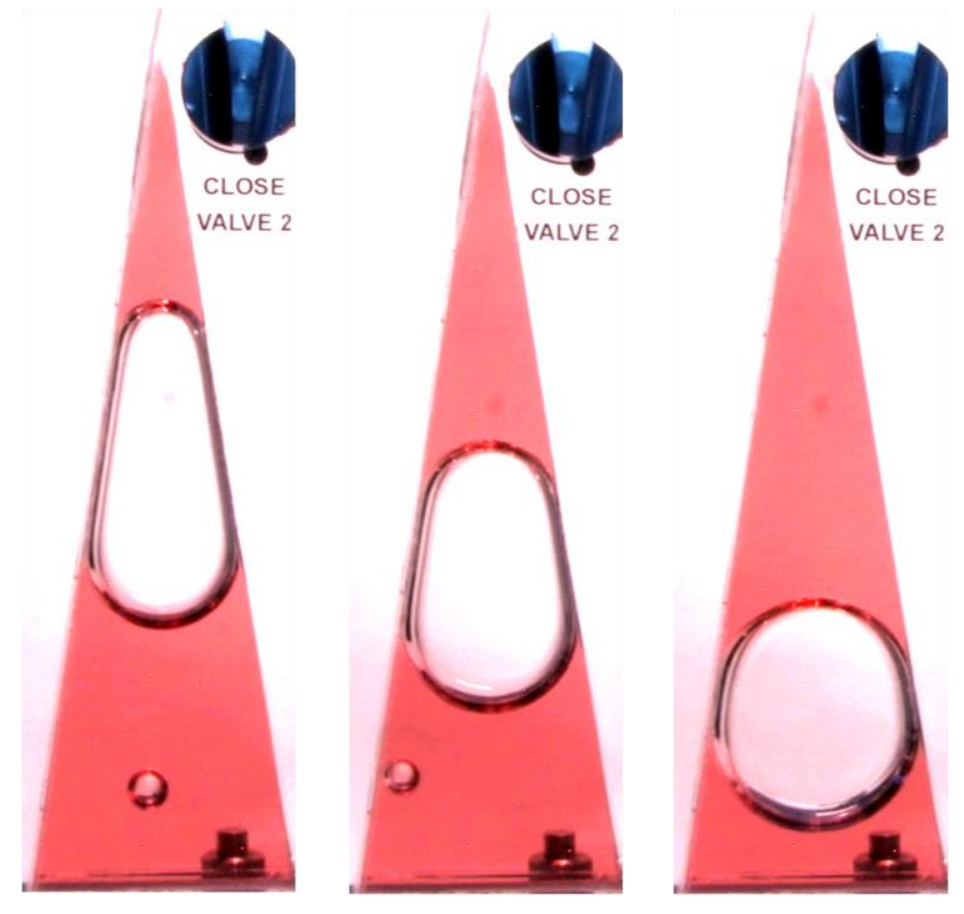

Figure 40. ICF2 25ml test: Image sequence progresses at approximately 3 minutes (middle) and 10 minutes of flow (right).

All data is recorded on flight DVCAM tapes which affords a high level of image resolution and precision data. Within a few days the tapes are downlinked to NASA Johnson Space Center (JSC) and standard-definition (SD) copies of the video are immediately available via a shared NASA GRC e-Room for initial analysis. However, high-definition (HD) versions are generated at JSC, burnt to Blu-ray discs, and are received by the investigator team within a few weeks of the experiments. The HD video has a resolution of 1920 by 1080 px at $29.97 \mathrm{fps}$ and has such clarity that steps must be taken during data collection to make sure that minute dust on the lens of the DVCAM has been cleared so that data is not obscured. The video files arrive in .m2ts format and are converted to an HD AVI format which is readable by NASA's Spotlight-16 image analysis software [35]. At this stage a Scale Factor Field (mm/pix) must be determined to 
ortho-rectify the data from the video frames so that distances between points can be accurately measured. The Scale Factor Field, seen in Figure 41, is constructed from several pairs of points on the ICF container with known distances from each other and which also lie in the $x, y$, and $z$ plane of the data collected. Conditions vary depending on how each astronaut chooses to set up the experiment, but the resolution of the Scale Factor Field is usually on the order of $0.10 \mathrm{~mm} / \mathrm{px}$. Leading and trailing bulk menisci locations, relative to the known virtual apex of the ICF1 tapered-isosceles test chamber, are measured during pre-wetted and wetted runs of the vessel. Time-dependent interface locations are then scaled into dimensionless forms as suggested by current theory for subsequent comparisons.

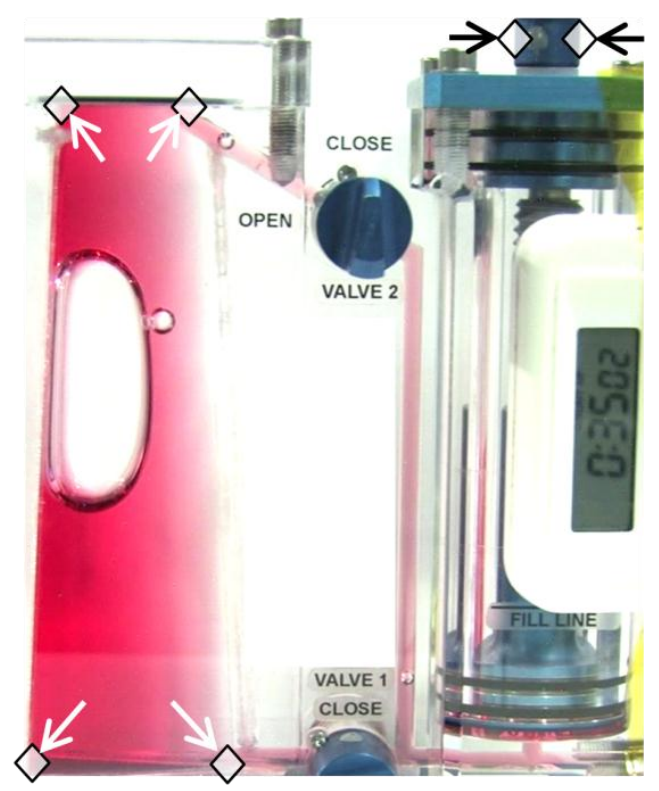

Figure 41. Scale factor points: 3 pairs of points are chosen with known distances from each other. The further away from each other the 3 pairs are, the more accurately the scale field can be determined. Also seen here is a timer (far right) which is used to account for loss of time stamp during HD downlink. Behind the timer is the fluid reservoir with piston fully dispensed. Running between the reservoir and test chamber are 2 transport tubes and valves used to deploy and drain fluid during experiments. 


\section{Vane Gap Experiments Bulk Shift Data}

Table 9. VG1 and VG2 Bulk Shift and Reverse Bulk Shift angles in degrees. All angles are measured from $\varphi$, the angle from $0^{\circ}$ to the smooth gap.

\begin{tabular}{|l|c|c|}
\cline { 2 - 3 } \multicolumn{1}{c|}{} & VG-1 & VG-2 \\
\hline $\begin{array}{c}\text { Bulk Shift } \\
\text { Point }\end{array}$ & $\varphi$ & $\varphi$ \\
\hline Q1 - BS & $59.5-63.5$ & $40-44$ \\
\hline Q2-RBS & 43.548 .5 & $44-46$ \\
\hline Q2-BS & $125-132$ & $127-134$ \\
\hline Q2-RBS & $135.5-137$ & 140 \\
\hline Q3-BS & $237-238$ & $221-225$ \\
\hline Q3-RBS & $223-227$ & $224-226$ \\
\hline Q4-BS & $313-316$ & $315-320$ \\
\hline Q4-RBS & $318-319$ & $318-320$ \\
\hline
\end{tabular}




\section{Appendix C}

\section{MAMS Measurements and Mapping}

Acceleration Measurement Details: The MAMS trimmed mean filter is designed to reject higher magnitude transients such as thruster firings and crew activity. The filtering procedure sorts the data by magnitude and calculates the deviation from a normal distribution using the $Q$ parameter determined by $Q=\left(U_{20 \%}-L_{20 \%}\right) /\left(U_{50 \%}-L_{50 \%}\right)$, where $U$ and $L$ imply upper and lower, respectively. The upper and lower percentages are determined from the sorted data set. $Q$ is used in turn to adaptively calculate the amount of data to trim from the tails:

$$
\delta(Q)= \begin{cases}0.05 & \text { when } Q \leq 1.75 \\ 0.05+\frac{0.35}{0.25}(Q-1.75) & \text { when } 1.75<Q<2 \\ 0.4 & \text { when } Q \geq 2\end{cases}
$$

The quasi-steady acceleration level is computed as the arithmetic mean of the trimmed set. Further information concerning the trimmed mean filter can be found in $[38,39,40]$.

The quasi-steady acceleration vector can be estimated at locations other than the MAMS sensor head location. The method used by MAMS assumes that the ISS vehicle is a rigid body in light of the quasi-steady regime. The three main contributions to the quasi-steady acceleration environment are aerodynamic drag, rotation, and gravity gradient effects. The drag effect is the same for any point attached to the vehicle and is considered to act opposite to the direction of flight. Rotational effects are the tangential and radial acceleration contributions due to the rotation of the vehicle. It is assumed that the accelerations in the tangential direction are negligible. Gravity gradient effects refer to accelerations acting at any point of the spacecraft away from the center of mass (CM). 
An independent particle will tend to gravitate towards the CM along $x$ - and $y$-axes of LVLH. This is a positive acceleration in the vehicle reference frame. A particle will gravitate away from the $\mathrm{CM}$ if it is above or below the CM along the $z_{\text {ISS-axis of LVLH }}$ [41]. Therefore, the LVLH acceleration acting on a particle within an orbiting vehicle can be written as:

$$
\begin{gathered}
\Delta \vec{g}\left(x_{L V}, y_{L V}, z_{L V}\right)=-\left|\omega_{o}\right|^{2}\left[x \hat{x}_{L V}+y \hat{y}_{L V}-2 z \hat{z}_{L V}\right]+\vec{a}_{\text {radial }}, \\
\vec{a}_{\text {radial }}=-\vec{\omega}_{O} \times \vec{\omega}_{O} \times \Delta \vec{r},
\end{gathered}
$$

where $x, y$, and $z$ are the distances to the ISS CM, and here subscript $L V$ refers to the LVLH axes. Here $\Delta g=\Delta g\left(x_{L V}, y_{L V}, z_{L V}\right)$ is the acceleration difference vector between the ISS CM and the measurement location, $\omega_{o}$ is the angular velocity vector of ISS, and $\Delta \boldsymbol{r}$ is the displacement vector from the ISS CM. The estimate of the quasi-steady acceleration at the new location $a_{n e w}$ is calculated by subtracting the correction at the MAMS location $a_{M A M S}$ and adding the effects at the new location such that

$$
\vec{a}_{\text {new }}=\vec{a}_{\text {MAMS }}-\Delta \vec{g}_{\text {MAMS }}+\Delta \vec{g}_{\text {new }} .
$$

Data used in these calculations, including vehicle center of mass, position, velocity and orientation was received through ISS telemetry available from the Marshall Spaceflight Center payload operations. Payload and center of rack positions were determined from ISS Design Analysis Cycle 8 values received from the ISS Structures and Mechanisms group. More details on MAMS data processing can be found in the ISS Increment 6/8 Microgravity Summary Report [42]. Figure 10 shows a sample of MAMS data during ICF1-4 after subtracting sensor bias. The mean acceleration during experiment operation can then be determined. 